\title{
molecules
}

ISSN 1420-3049

www.mdpi.com/journal/molecules

Article

\section{Design and Synthesis of a Novel Ganglioside Ligand for Influenza A Viruses ${ }^{\dagger}$}

Tomohiro Nohara ${ }^{1}$, Akihiro Imamura ${ }^{1,2, *}$, Maho Yamaguchi ${ }^{3}$, Kazuya I. P. J. Hidari ${ }^{3}$, Takashi Suzuki ${ }^{3}$, Tatsuya Komori ${ }^{1}$, Hiromune Ando ${ }^{1,2}$, Hideharu Ishida ${ }^{1}$ and Makoto Kiso ${ }^{1,2, *}$

1 Department of Applied Bioorganic Chemistry, Gifu University, 1-1 Yanagido, Gifu-shi, Gifu 501-1193, Japan; E-Mails: noharah18@yahoo.co.jp (T.N.); komorih17@yahoo.co.jp (T.K.); hando@gifu-u.ac.jp (H.A.); ishida@gifu-u.ac.jp (H.I.)

2 Institute for Integrated Cell-Material Sciences, Kyoto University, 69 Konoe-cho, Yoshida, Sakyo-ku, Kyoto 606-8501, Japan

3 Department of Biochemistry, School of Pharmaceutical Sciences, University of Shizuoka, 52-1 Yada, Suruga-ku, Shizuoka-shi, Shizuoka 422-8526, Japan; E-Mails: d11107@u-shizuoka-ken.ac.jp (M.Y.); hidari@u-shizuoka-ken.ac.jp (K.I.P.J.H.); suzukit@u-shizuoka-ken.ac.jp (T.S.)

$\dagger$ Synthetic studies on sialoglycoconjugates, Part 157.

* Authors to whom correspondence should be addressed; E-Mails: aimamura@gifu-u.ac.jp (A.I.); kiso@gifu-u.ac.jp (M.K.); Tel.: +81-58-293-3453 (A.I.); Fax: +81-58-293-2918 (A.I.).

Received: 9 July 2012; in revised form: 6 August 2012 / Accepted: 8 August 2012 /

Published: 10 August 2012

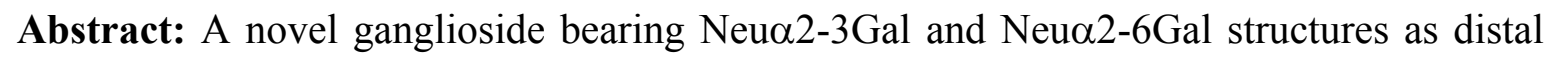
sequences was designed as a ligand for influenza A viruses. The efficient synthesis of the designed ganglioside was accomplished by employing the cassette coupling approach as a key reaction, which was executed between the non-reducing end of the oligosaccharide and the cyclic glucosylceramide moiety. Examination of its binding activity to influenza A viruses revealed that the new ligand is recognized by Neua2-3 and 2-6 type viruses.

Keywords: ganglioside; sialic acid; total synthesis; glycosylation; influenza virus 


\section{Introduction}

Influenza viruses cause a substantial number of deaths during annual epidemics and occasional pandemics $[1,2]$. Based on the antigenicity of their internal proteins the viruses are divided into three types, A, B, and C, of which either type A or B viruses cause seasonal influenza in humans. When the viruses bind to the host cell, hemagglutinin (HA) on their cell surface plays a significant role in the infection process. The HA protein recognizes sialoglycoconjugates expressed on the plasma membrane of the host cell, for example, sialoglycoproteins and gangliosides (sialoglycosphingolipids), as cellular ligands. Furthermore, HA can also recognize specific linkages between sialic acid (Neu5Ac/Gc) and lactosamine (LacNAc: Gal $31-4 \mathrm{GlcNAc}$ ) residues, which are found at the terminal end of glycoconjugates $[3,4]$. The structure and distribution of sialoglycans are crucial for viruses to determine their host animals, and two major linkage types, that is, Neu5Aco2-3LacNAc and Neu5Aca2-6LacNAc, are essential for viral transmission. Human and swine viruses predominantly recognize the Neu5Aco2-6LacNAc sequence, while avian and equine viruses bind preferentially to the Neu5Aco2-3Gal (including Neu5Aco2-3LacNAc) moiety. Swine are considered as intermediate hosts between humans and birds since they possess an abundance of both Neu5Aco2-3LacNAc and Neu5Aca2-6LacNAc structures as receptor carbohydrate determinants. The simultaneous infection of an intermediate host, such as swine, with avian and human viruses could lead to genetic recombination between the viruses, resulting in the generation of a new pathogenic virus that could potentially cause severe pandemics. However, the exact natural ligand for influenza A viruses in an intermediate host, such as pigs, remains unclear. Therefore, in this study, we focused on identifying a new carbohydrate ligand that was not only highly recognized by influenza A viruses but also functions as a natural receptor for viral HA. For this purpose, a ganglioside bearing both the Neu5Aco2-3 and Neu5Aca26LacNAc sequences was designed (Figure 1). It was hypothesized that the designed ganglioside 1 could be recognized by human- and avian-derived viruses because it contains two types of sialoglycan in a single molecule. We report the chemical synthesis of ganglioside $\mathbf{1}$ and its binding activity to influenza A viruses.

\section{Results and Discussion}

\subsection{Chemical Synthesis}

It was envisaged that the efficient synthesis of $\mathbf{1}$ could be achieved using the cassette approach between the non-reducing end of the oligosaccharide and the glucosylceramide, which was recently developed by our group [5-10]. Furthermore, it was thought that the construction of the non-reducing end of the heptasaccharide moiety, which includes two types of sialoside, Neu5Aco2-3/2-6Gal, should be executed through a convergent synthetic approach. For the convergent synthesis of a relatively large oligosaccharide such as $\mathbf{1}$, the design of the building blocks often affects the efficiency of the total synthesis as well as the overall yield. Our preliminary experiment on the synthesis of a ganglioside similar to 1 gave a significant finding that monosaccharyl (GlcN) units are more useful as glycosyl donors than oligosaccharyl (Neu5Ac $\alpha 2-3 / 2-6 \mathrm{Gal} \beta 1-4 \mathrm{GlcN}$ ) donors for the formation of branched structure at the 3-and 6-positions of the inner galactose residue (data not shown). Therefore, target 1 
was divided into four major components, from which each building block (Units A-D) was designed (Figure 1).

Figure 1. Structures of the target ganglioside 1 and the designed building blocks (Units A-D).

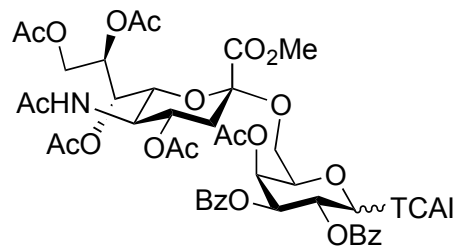

Unit B

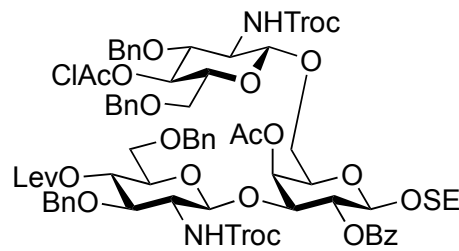

Unit C

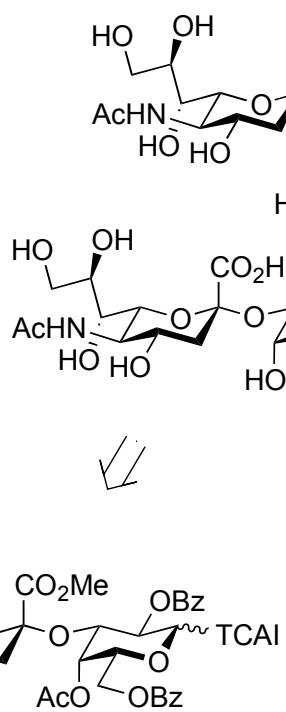

Unit A

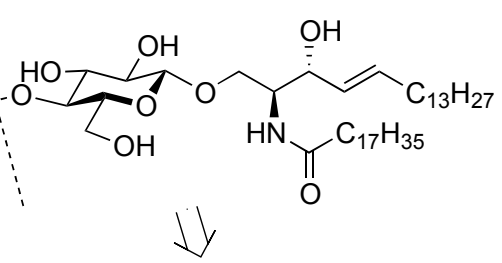

1

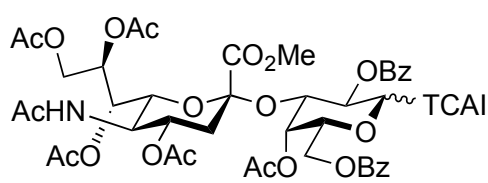

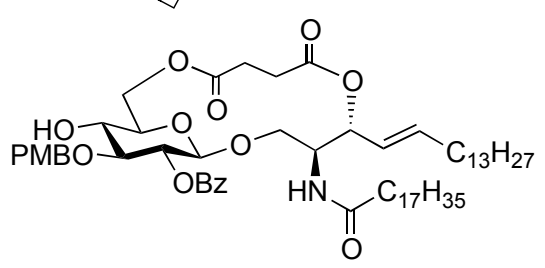

Unit D

The synthetic method for the terminal Neu5Aca2-3Gal unit $\mathbf{A}$ has been already established by our group. The coupling of the 5-N-Troc-protected sialyl donor $\mathbf{2}$ and galactosyl acceptor $\mathbf{3}$, carrying a $p$-methoxyphenyl (MP) group at the anomeric position, generated the Neu5Troc $\alpha 2-3 \mathrm{Gal}$ disaccharide in good yield. The isolation of $\alpha$-sialoside from the reaction mixture was easily accomplished by recrystallization [11]. The obtained disaccharide was readily converted into the corresponding trichloroacetimidate donor as Unit A [12]. Similarly, the other terminal Neu5Aco2-6Gal unit (B) was prepared efficiently according to the synthetic procedure for Unit A (Scheme 1). The sialylation of the diol galactosyl acceptor 5 was performed in the presence of NIS and TfOH $[13,14]$ in a mixed solvent system, propionitrile-dichloromethane $(5: 1)$, at $-30{ }^{\circ} \mathrm{C}[15,16]$. This mixed solvent system was used because of the poor solubility of the acceptor 5 in acetonitrile. In addition, temperatures lower than $-30{ }^{\circ} \mathrm{C}$ led to a significant decrease in the yield, possibly because of the observed precipitation of $\mathbf{5}$ during the reaction. As a result of optimization, the desired $\alpha$-glycoside 6 was obtained in $69 \%$ yield along with a $14 \%$ yield of the $\beta$-isomer. Purification of the $\alpha$-glycoside 6 by silica gel column chromatography was troublesome compared with that of the regioisomer, Neu5Troca2-3GalMP, which has benzyl groups on the O-2 and O-6 positions of its galactose residue, which can be isolated easily by recrystallization from an EtOAc/ $n$-hexane system [12]. The selective deprotection of the Troc group with $\mathrm{Zn}-\mathrm{Cu}$ [17] (giving 7) and the subsequent concomitant acetylation of the liberated amine 
and the hydroxyl group at the C-4 position of the galactose residue afforded compound $\mathbf{8}$ in excellent yield. Hydrogenolysis of the benzyl groups over Pearlman's catalyst in 1,4-dioxane followed by benzoylation generated compound $\mathbf{1 0}$ in $94 \%$ yield over two steps. The exposure of $\mathbf{1 0}$ to CAN and $\mathrm{H}_{2} \mathrm{O}$ [18] (giving 11) and the subsequent introduction of a trichloroacetimidate group [19] afforded the Neu5Aca2-6Gal donor 12 (Unit B) in 63\% yield over two steps.

Scheme 1. Efficient synthesis of Units A and B from the $N$-Troc-protected sialyl donor 2.

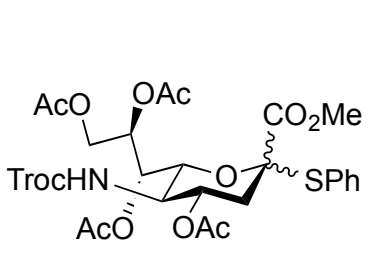

2

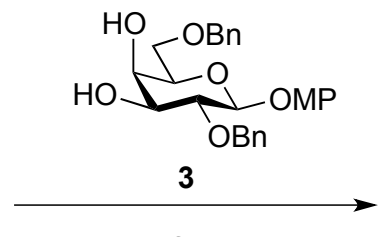

Ref. 11,12

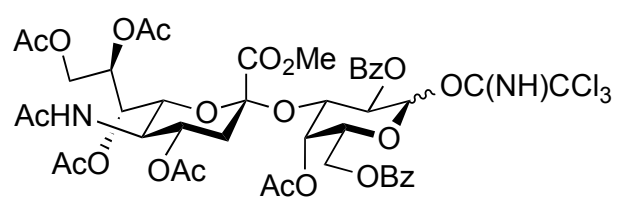

4

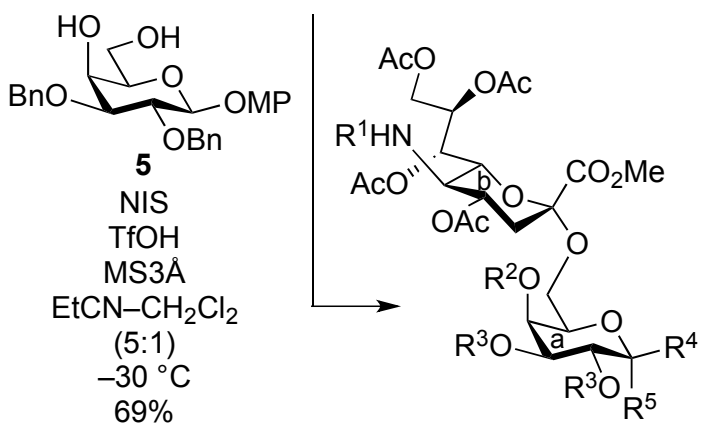

(Unit A)

Reagents and Conditions: (a) $\mathrm{Zn}-\mathrm{Cu}, \mathrm{AcOH}-\mathrm{CH}_{2} \mathrm{Cl}_{2}$ (3:2), $40{ }^{\circ} \mathrm{C}$; (b) $\mathrm{Ac}_{2} \mathrm{O}$, DMAP, Py, r.t.; (c) $\mathrm{H}_{2}, \mathrm{Pd}(\mathrm{OH})_{2} / \mathrm{C}, 1,4-$ dioxane, r.t.; (d) $\mathrm{Bz} 2 \mathrm{O}$, DMAP, Py, r.t.; (e) CAN, MeCN-PhMe- $\mathrm{H}_{2} \mathrm{O}$ (6:5:3), r.t.; (f) $\mathrm{CCl}_{3} \mathrm{CN}, \mathrm{DBU}, \mathrm{CH}_{2} \mathrm{Cl}_{2}, 0^{\circ} \mathrm{C}$.

The inner core trisaccharide structure, Unit C, was prepared starting from the 2- $N$-Troc protected glucosamine derivative 13 (Schemes 2 and 3). First, the glucosaminyl donors 16 and 17 were prepared as shown in Scheme 2. Removal of the acetyl groups from $\mathbf{1 3}$ and the subsequent formation of cyclic benzylidene acetal between O-4 and O-6 afforded 14 in good yield. The following benzylation step in the presence of a Troc group was conducted under reductive conditions. Optimization of this reductive benzylation with benzaldehyde, TESOTf, and triethylsilane [20] revealed that the use of toluene as a solvent could increase the yield. The successive reductive opening of the benzylidene group by treatment with $\mathrm{BF}_{3}$ etherate and triethylsilane [21] gave $\mathbf{1 5}$ in 78\% yield over two steps from $\mathbf{1 4}$. The obtained alcohol 15 was transformed into two types of glucosaminyl donors, namely, 16 and 17, via the introduction of a levulinoyl (Lev) and monochloroacetyl (ClAc) group to the hydroxyl group at $\mathrm{C}-4$, respectively. 
Scheme 2. Preparation of glucosaminyl donors 16 and 17 towards Unit C.

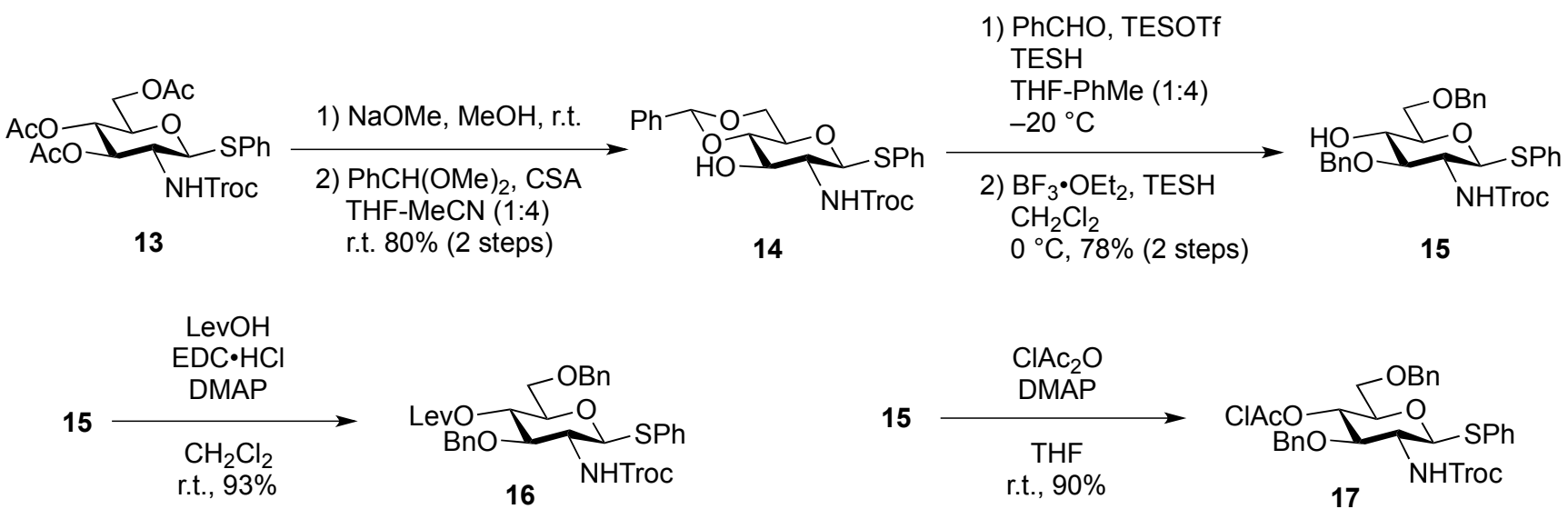

Scheme 3. Assembly of the inner core fragment, Unit C.
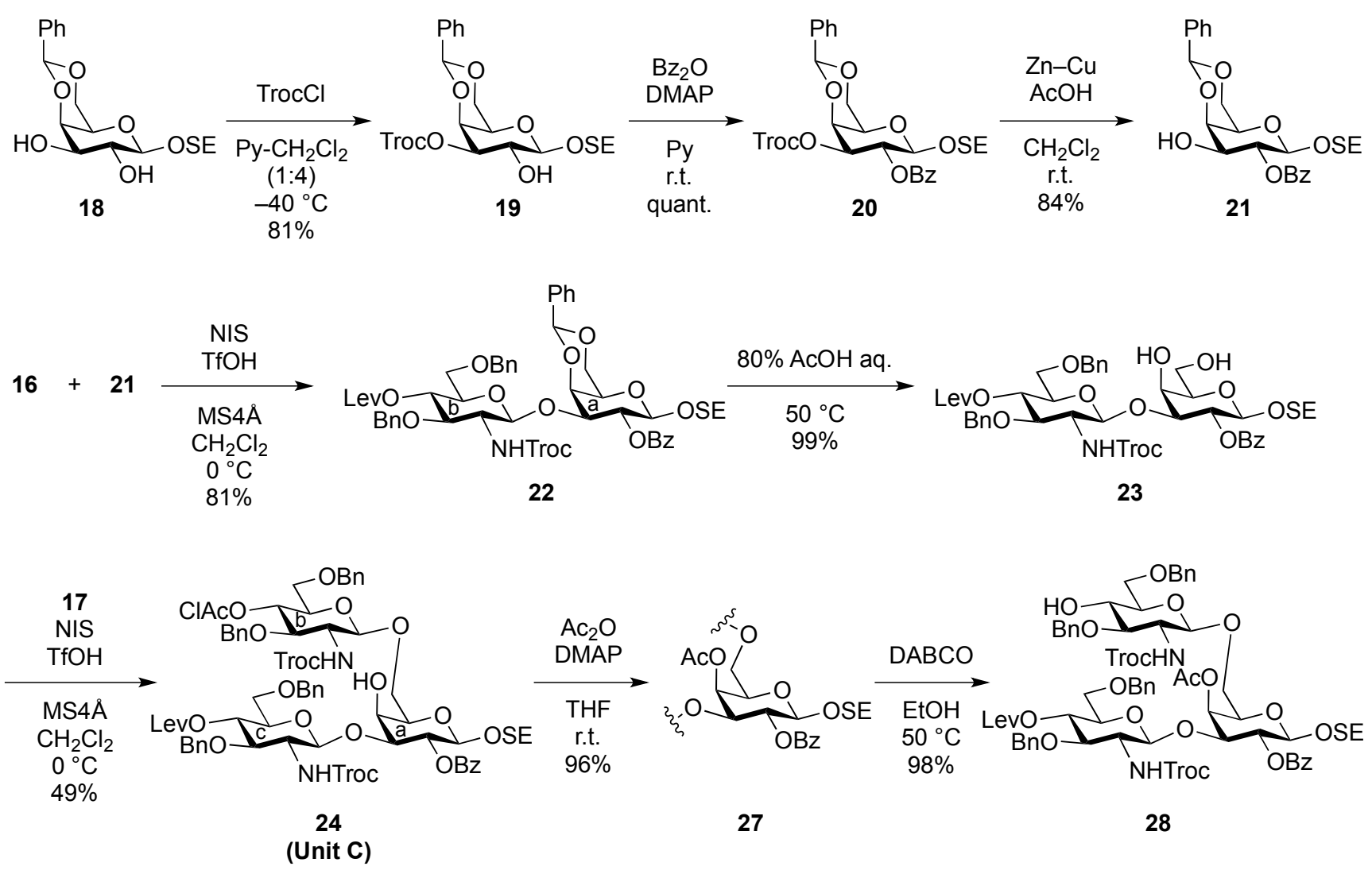

The glucosaminyl donors 16 and $\mathbf{1 7}$ were then incorporated into the galactosyl acceptor 21, which was prepared readily from the known galactose derivative 18 [22] via three steps. Selective protection of the hydroxyl group at C-3 by the Troc group was carried out under basic conditions with TrocCl at a low temperature $\left(-40^{\circ} \mathrm{C}\right)$ to afford 19 in $81 \%$ yield. Benzoylation under standard conditions (giving 20) and the selective removal of the Troc group with $\mathrm{Zn}-\mathrm{Cu}$ furnished the galactosyl acceptor $\mathbf{2 1}$ in good yield. The obtained 21 was then subjected to glycosylation with donor 16 in the presence of NIS and TfOH in $\mathrm{CH}_{2} \mathrm{Cl}_{2}$ at $0{ }^{\circ} \mathrm{C}$, affording the disaccharide 22 in $81 \%$ yield. The stereochemical assignment was confirmed by ${ }^{1} \mathrm{H}-\mathrm{NMR}$, where the $J_{1,2}$ value of $7.6 \mathrm{~Hz}$ for $\mathrm{H}-1$ indicated the $\beta$-configuration of the glucosamine residue. Next, hydrolysis of the benzylidene group with $80 \% \mathrm{AcOH}$ aq at $50{ }^{\circ} \mathrm{C}$ afforded 
the diol 23 at an almost quantitative yield. A second round of glucosaminidation was conducted between 17 and 23 under the same conditions as those of the initial glucosaminidation between $\mathbf{1 6}$ and 21. As a result, the desired trisaccharide 24 was obtained as Unit $\mathbf{C}$ in a moderate yield of $49 \%$. In this reaction, a non-negligible amount of the tetrasaccharide 25, in which both hydroxyl groups were glucosaminylated, was observed as a byproduct (Figure 2). An attempt at using a lower temperature to increase the selectivity failed due to the poor solubility of acceptor $\mathbf{2 3}$ in $\mathrm{CH}_{2} \mathrm{Cl}_{2}$. Furthermore, changing the other factors for glycosylation, for example, the leaving group (using trichloroacetimidate) and how the donor was added, did not improve the yield of $\mathbf{2 4}$. It is of importance that the generation of the tetrasaccharide $\mathbf{2 5}$ during the reaction was faster than the complete consumption of the acceptor 23. In addition, the trisaccharide 26, which was glucosaminylated at C-4 of the galactose residue, was not detected among the by-products. These findings suggested that the newly formed trisaccharyl alcohol 24 was preferred to the disaccharyl acceptor $\mathbf{2 3}$ as a glycosyl acceptor. This phenomenon might be explained by the poor solubility of the disaccharyl alcohol 23 in $\mathrm{CH}_{2} \mathrm{Cl}_{2}$ compared with the trisaccharyl alcohol 24 (Figure 2). Next, the acetylation of $\mathbf{2 4}$ with acetic anhydride and DMAP in THF [23] was carried out to protect the free hydroxyl group, affording 27 in 96\% yield (Scheme 3). The monochloroacetyl group on $\mathbf{2 7}$ was then unblocked using DABCO in ethanol [24] with an excellent yield, providing the inner core trisaccharide acceptor 28, which was ready for the next glycosylation step.

Figure 2. Explanation for the poor regioselectivity observed during glucosaminylation.

Lower solubility in $\mathrm{CH}_{2} \mathrm{Cl}_{2}$

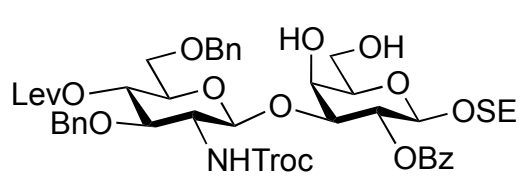

23

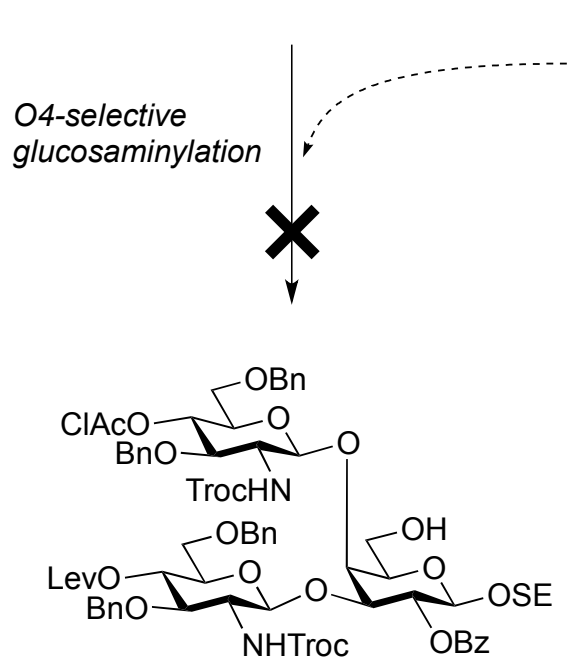

26

(Not detected)
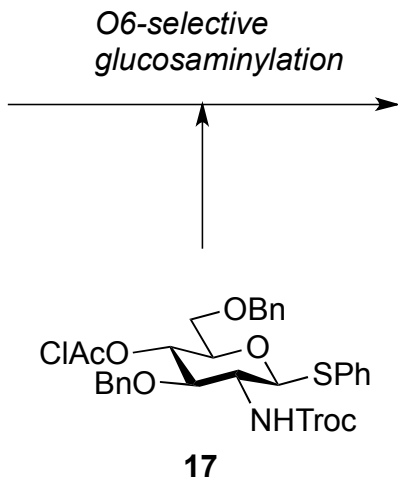

17

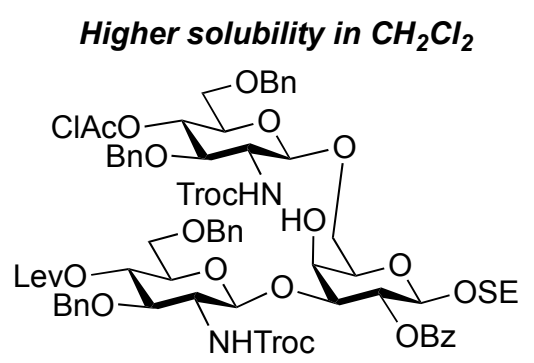

24

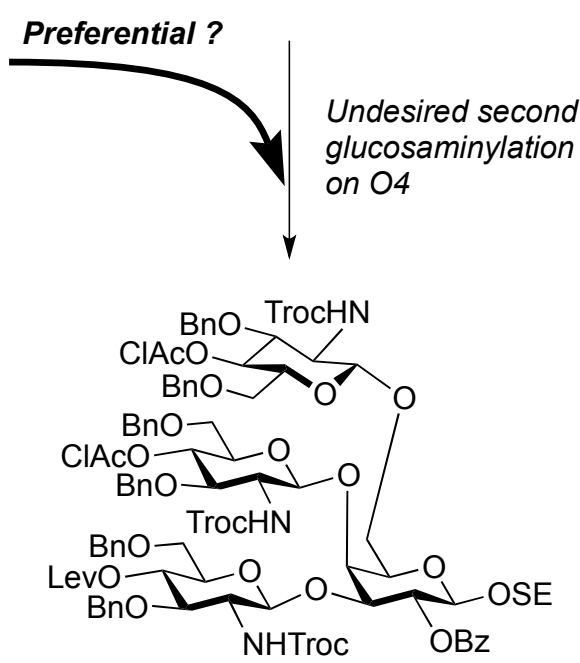

25 
As depicted in Scheme 4, the coupling of the trisaccharide acceptor 28 with the Neu5Aca2-6Gal donor 12 promoted by TMSOTf was conducted in $\mathrm{CH}_{2} \mathrm{Cl}_{2}$ at room temperature, affording the pentasaccharide 29 in 74\% yield. During this glycosylation step, the generation of several by-products containing trichloroacetamide glycoside, which is occasionally formed as a by-product during glycosylation using trichloroacetimidate donors, made the purification process an arduous task. Column chromatography on silica gel followed by gel filtration was found to be useful for purification. Next, the conversion of the Troc carbamate at C-2 of both glucosamine residues into acetamide was achieved by treatment of alloyed zinc with copper in $\mathrm{AcOH}$ and followed by acetylation, giving the acetamide compound 31 in 61\% yield over two steps. Finally, cleavage of the levulinoyl group by using hydrazine monoacetate in THF [25] released the 4-OH to provide the pentasaccharide acceptor 32 in $92 \%$ yield.

Scheme 4. Coupling of Units $\mathbf{A}$ and $\mathbf{C}$ followed by transformation of the corresponding acceptor.

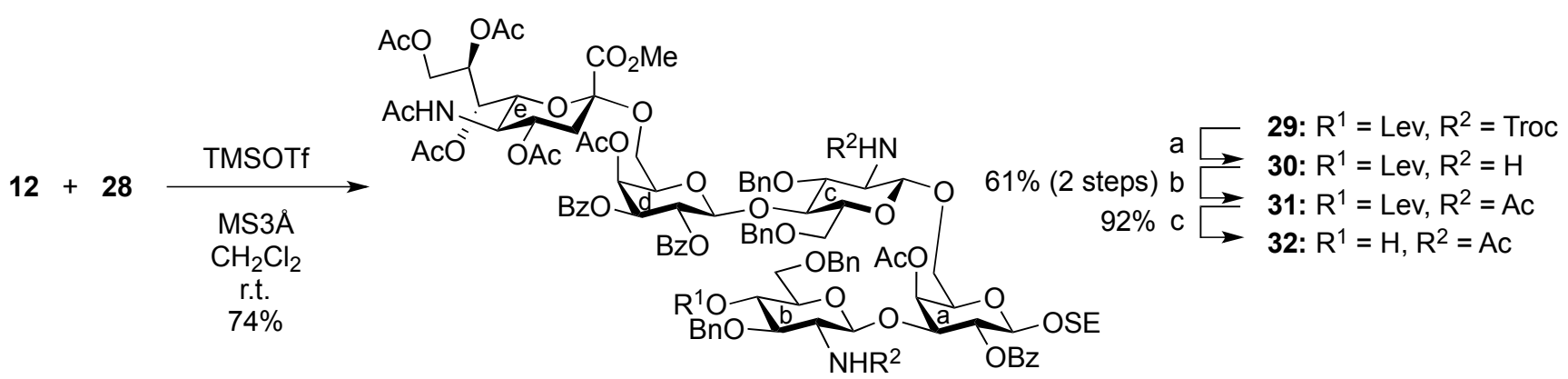

Reagents and Conditions: (a) $\mathrm{Zn}-\mathrm{Cu}, \mathrm{AcOH}-\left(\mathrm{CH}_{2} \mathrm{Cl}_{2}\right)_{2}$ (3:2), $50{ }^{\circ} \mathrm{C}$; (b) $\mathrm{Ac}_{2} \mathrm{O}$, DMAP, Py, r.t.; (c) $\mathrm{NH}_{2} \mathrm{NH}_{2} \cdot \mathrm{AcOH}$, THF, r.t.

Scheme 5 shows the assembly of the non-reducing end heptasaccharide moiety. The Neu5Aca2$3 \mathrm{Gal}$ donor 4 was coupled with 32 in the presence of TMSOTf in $\mathrm{CH}_{2} \mathrm{Cl}_{2}$ at room temperature to provide the heptasaccharide 33 in $62 \%$ yield. During this glycosylation step, the generation of the trichloroacetamide glycoside and the dimer of donor 4 , which was formed by the nucleophilic attack of the hydrolyzed donor on the oxocarbenium species derived from the donor, as by-products, made the purification of the desired product $\mathbf{3 3}$ laborious. The structure of isolated $\mathbf{3 3}$ was elucidated based on its MS, ${ }^{1} \mathrm{H}$, and ${ }^{13} \mathrm{C}$-NMR spectra. For instance, the $\beta$-configuration of the newly formed glycosidic linkage was evident from the coupling constant of the anomeric proton at $\delta 5.01\left(J_{1,2}=7.5 \mathrm{~Hz}\right)$. Next, cleavage of the benzyl groups by hydrogenolysis (giving 34) followed by acetylation with conventional conditions afforded 35 in 89\% yield over two steps. Selective exposure of the anomeric hydroxyl group was easily achieved by treatment with trifluoroacetic acid in $\mathrm{CH}_{2} \mathrm{Cl}_{2}$ to yield 36. This was then converted to the corresponding trichloroacetimidate donor $\mathbf{3 7}$ in $95 \%$ yield over two steps from 35, which was then ready for cassette coupling with the glucosylceramide block 38 (Unit D). 
Scheme 5. Synthesis of the non-reducing end heptasaccharide part.

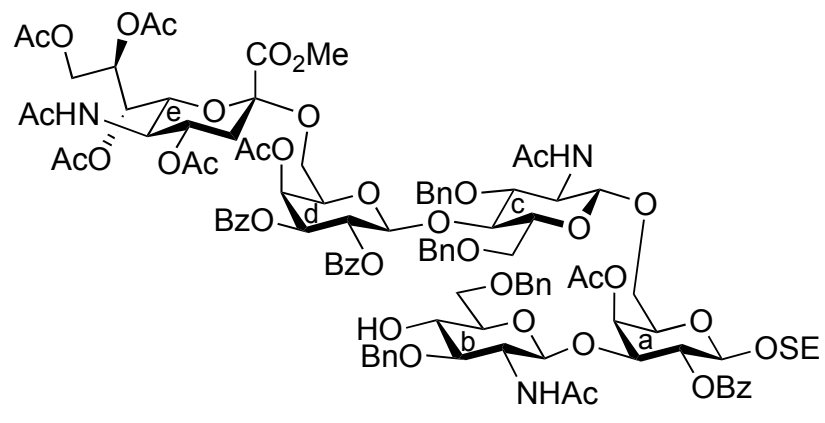

32
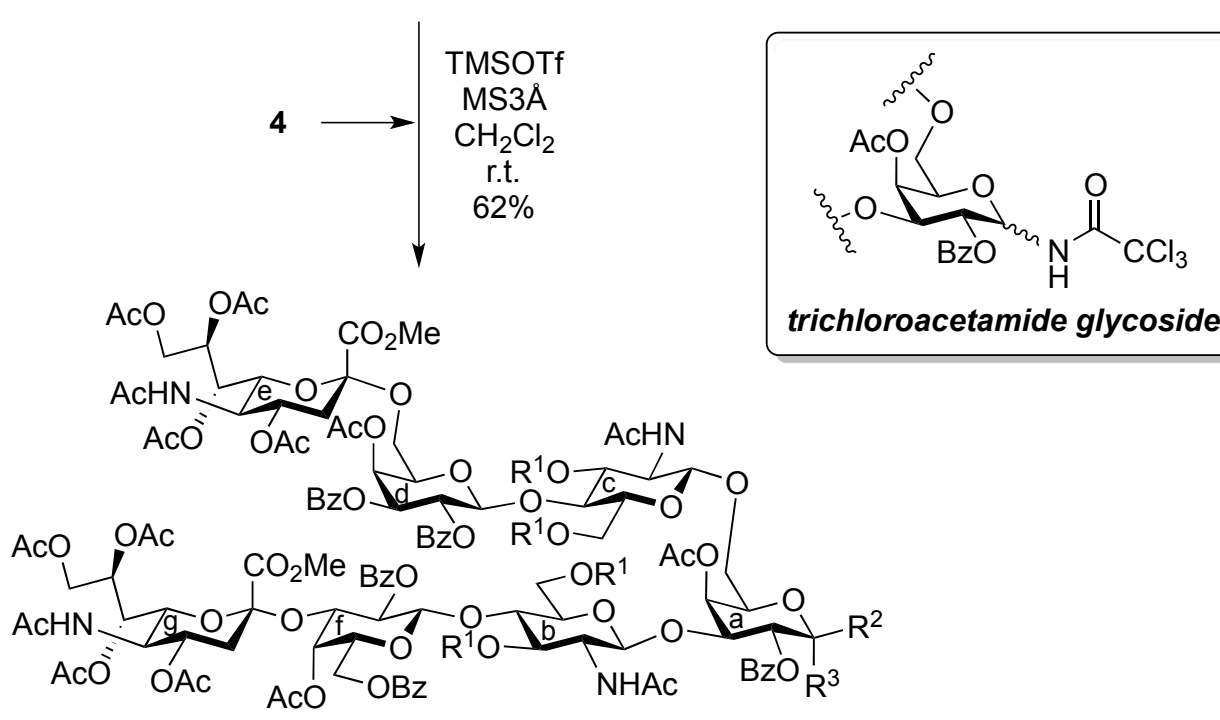

$\begin{aligned} & \text { a } \text { 33: } R^{1}=\mathrm{Bn}, \mathrm{R}^{2}=\mathrm{OSE}, \mathrm{R}^{3}=\mathrm{H} \\ & \text { 89\% (2 steps) b } \square \text { 34: } \mathrm{R}^{1}=\mathrm{H}, \mathrm{R}^{2}=\mathrm{OSE}, \mathrm{R}^{3}=\mathrm{H} \\ & \text { c } \square \text { 35: } \mathrm{R}^{1}=\mathrm{Ac}, \mathrm{R}^{2}=\mathrm{OSE}, \mathrm{R}^{3}=\mathrm{H} \\ & \text { 95\% (2 steps) d } \longrightarrow \text { 36: } \mathrm{R}^{1}=\mathrm{Ac}, \mathrm{R}^{2}, \mathrm{R}^{3}=\mathrm{H}, \mathrm{OH} \\ & \longrightarrow 37: \mathrm{R}^{1}=\mathrm{Ac}, \mathrm{R}^{2}, \mathrm{R}^{3}=\mathrm{H}, \mathrm{OC}(\mathrm{NH}) \mathrm{CCl}_{3}\end{aligned}$

Reagents and Conditions: (a) $\mathrm{H}_{2}, \mathrm{Pd}(\mathrm{OH})_{2} / \mathrm{C}, 1$,4-dioxane, r.t.; (b) $\mathrm{Ac}_{2} \mathrm{O}$, DMAP, Py, r.t.; (c) TFA, $\mathrm{CH}_{2} \mathrm{Cl}_{2}, 0{ }^{\circ} \mathrm{C}$; (d) $\mathrm{CCl}_{3} \mathrm{CN}, \mathrm{DBU}, \mathrm{CH}_{2} \mathrm{Cl}_{2}, 0{ }^{\circ} \mathrm{C}$.

We previously addressed the development of the cassette coupling approach between a non-reducing end oligosaccharide and a glucosylceramide (GlcCer) moiety for the synthesis of various glycolipids, particularly gangliosides [5-10]. This approach resulted in a solution to the inevitable low yield of sugar and ceramide fragments. Hitherto, we developed two types of GlcCer units: one is a cyclic type GlcCer tethered by succinic ester between the sugar and lipid portions [5,7,8], while the other is an acyclic type GlcCer $[6,9,10]$. In this study, we chose the cyclic type due to its ease of preparation. The reported cyclic GlcCer acceptor 38 [7] was subjected to glycosylation with the oligosaccharide donor 37 in the presence of TMSOTf in $\mathrm{CHCl}_{3}$ at room temperature, affording the fully protected ganglioside 39 in a moderate yield of $49 \%$. In this reaction, chloroform was employed as solvent instead of the conventional dichloromethane because of the somewhat poor solubility of $\mathbf{3 8}$ in $\mathrm{CH}_{2} \mathrm{Cl}_{2}$. Following the same protocol as that used for the other glycosylation reactions, the structure of $\mathbf{3 9}$ was elucidated. Next, cleavage of the $p$-methoxybenzyl (PMB) group by TFA in $\mathrm{CH}_{2} \mathrm{Cl}_{2}$ at $0{ }^{\circ} \mathrm{C}$ provided 40 in $90 \%$ 
yield. Finally, global deprotection under Zemplén conditions followed by saponification generated the target ganglioside 1 in good yield (Scheme 6).

Scheme 6. Final coupling by the cassette approach followed by global deprotection.
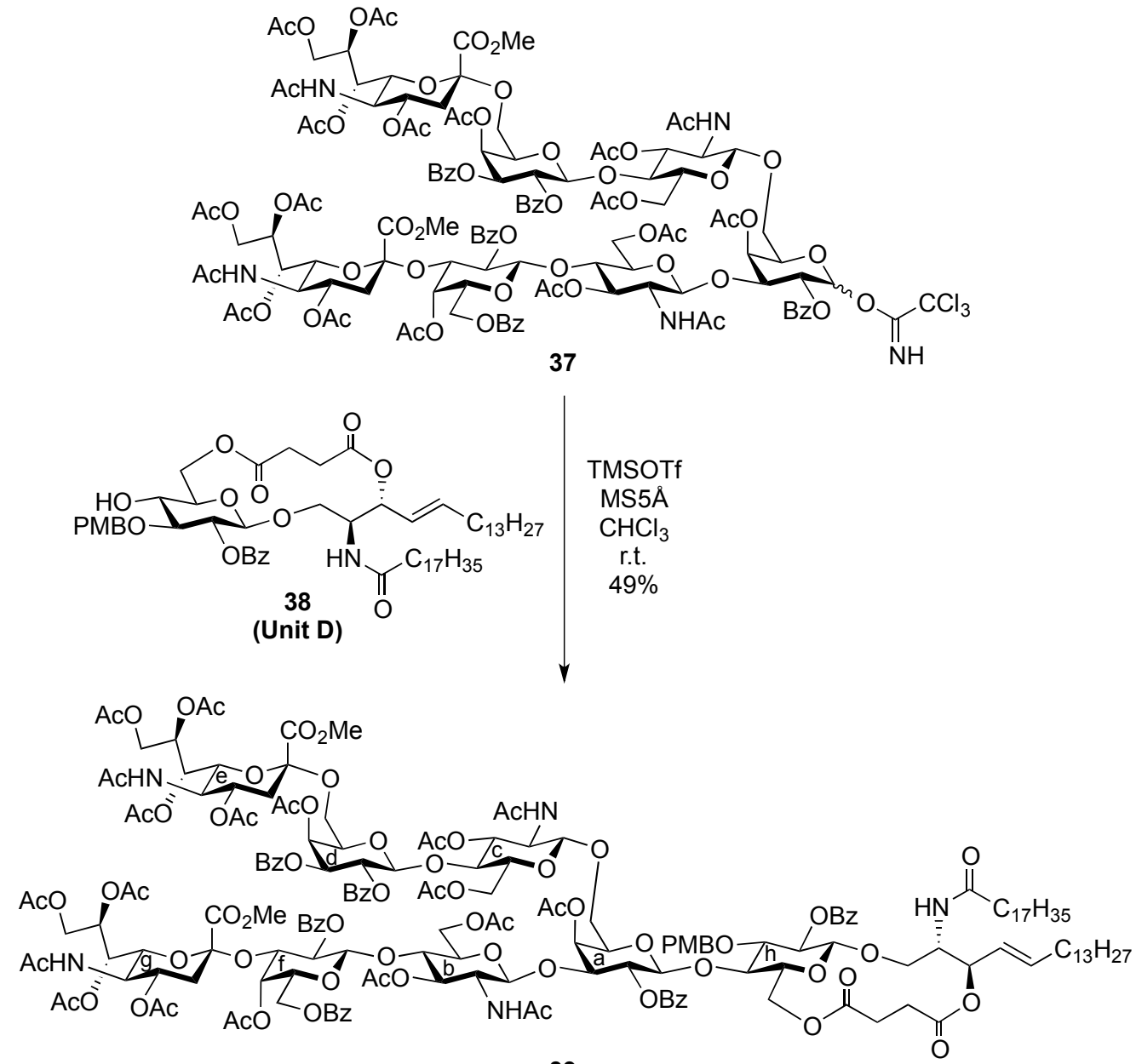

39

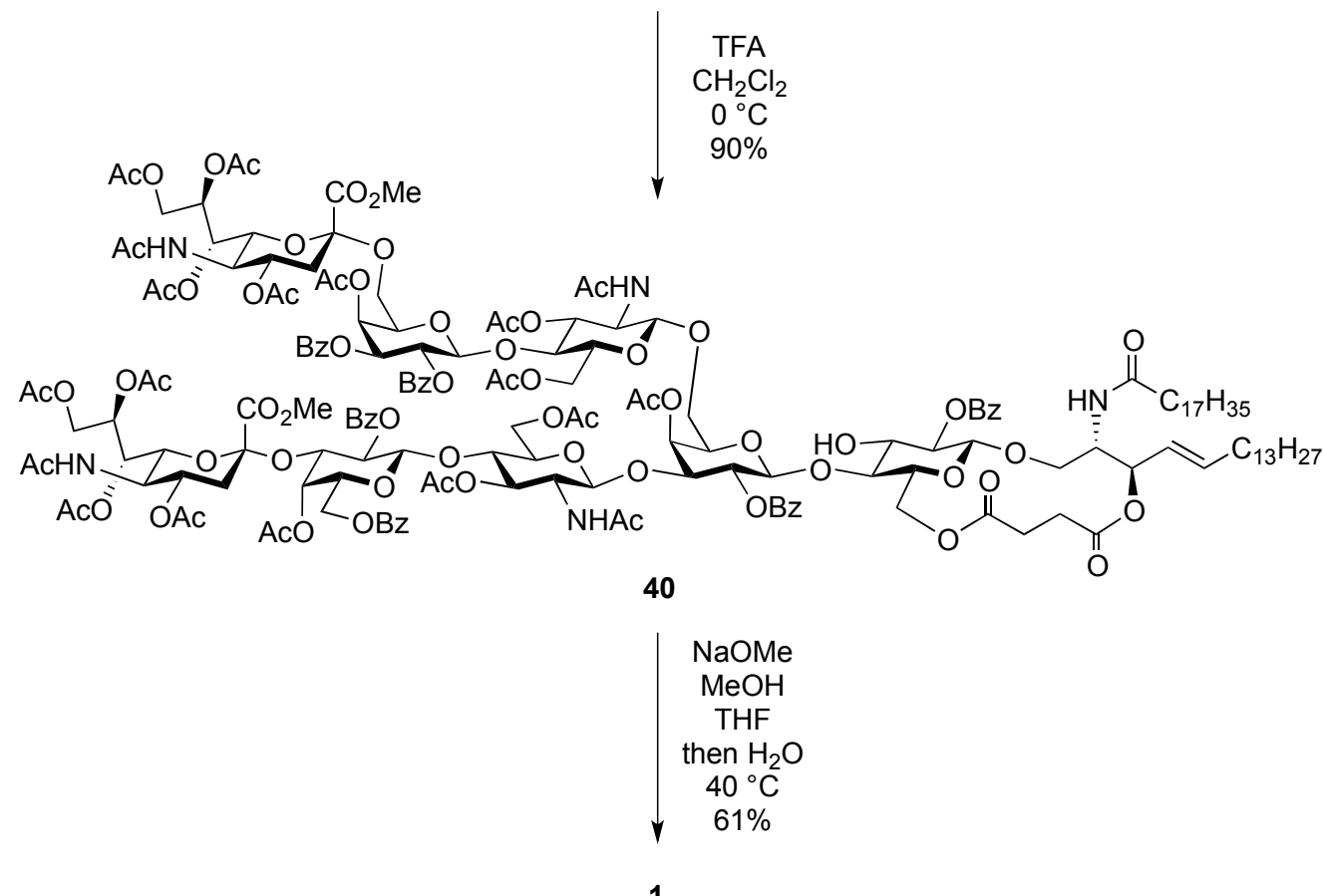




\subsection{Binding Assay}

The synthesized ganglioside 1 (termed GSC-734) was then assessed for its binding activity to influenza viruses (Figure 3). The binding assay showed that GSC-734 was recognized by Neu5Ac $\alpha 2-3$ and 2-6 type viruses. Moreover, the binding activity of the Neu5Aca2-6 type virus (H3N2) was almost identical to that of the previously reported $\alpha 2-6$ sialylparagloboside [26]. This observation suggests that the branched structure of the sugar part does not potently influence its binding activity to the viruses.

Figure 3. Binding activity of the synthetic ligand (GSC-734) for influenza A viruses.
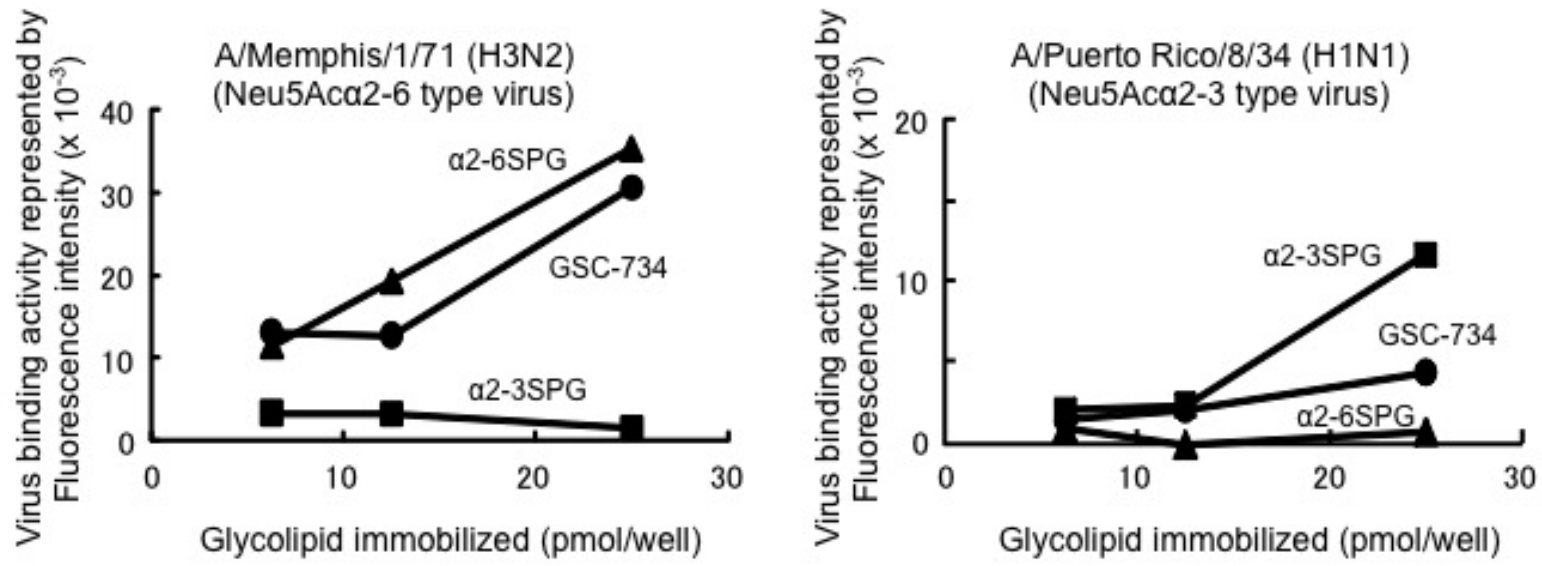

\section{Experimental}

\subsection{General Methods for Chemical Synthesis}

All reactions were carried out under a positive pressure of argon, unless otherwise noted. All chemicals were purchased from commercial suppliers and used without further purification, unless otherwise noted. Molecular sieves were purchased from Wako Chemicals Inc. (Osaka, Japan) and dried at $300{ }^{\circ} \mathrm{C}$ for $2 \mathrm{~h}$ in a muffle furnace prior to use. Solvents as reaction media were dried over molecular sieves and used without purification. TLC analysis was performed on Merck TLC (silica gel 60F254 on glass plate, Darmstadt, Germany). Compound detection was either by exposure to UV light (2536 $\AA$ ) or by soak in a solution of $10 \% \mathrm{H}_{2} \mathrm{SO}_{4}$ in ethanol followed by heating. Silica gel ( 80 mesh and $300 \mathrm{mesh}$ ) manufactured by Fuji Silysia Co. (Kasugai, Japan) was used for flash column chromatography. Quantity of silica gel was usually estimated as 100 to 150 -fold weight of sample to be charged. Solvent systems in chromatography were specified in $v / v$. Evaporation and concentration were carried out in vacuo. ${ }^{1} \mathrm{H}-\mathrm{NMR}$ and ${ }^{13} \mathrm{C}-\mathrm{NMR}$ spectra were recorded with JEOL ECA $400 / 500 / 600$ spectrometers. Chemical shifts in ${ }^{1} \mathrm{H}-\mathrm{NMR}$ spectra are expressed in ppm $(\delta)$ relative to the signal of $\mathrm{Me}_{4} \mathrm{Si}$, adjusted to $\delta 0.00 \mathrm{ppm}$. Data are presented as follow: Chemical shift, multiplicity ( $\mathrm{s}=$ singlet, $\mathrm{d}=$ doublet, $\mathrm{t}=$ triplet, $\mathrm{dd}=$ double of doublet, $\mathrm{dt}=$ double of triplet, $\mathrm{m}=$ multiplet and/or multiple resonances), integration, coupling constant in $\mathrm{Hertz}(\mathrm{Hz})$, position of the corresponding proton. COSY methods were used to confirm the NMR peak assignments. MALDI-TOF mass spectra were run in a Bruker Autoflex (Billerica, MA, USA) and CHCA was used as the matrix. Highresolution mass (ESI-TOF MS) spectra were run in a Bruker micrOTOF. Optical rotations were measured with a 'Horiba SEPA-300' high-sensitive polarimeter (Kyoto, Japan). 


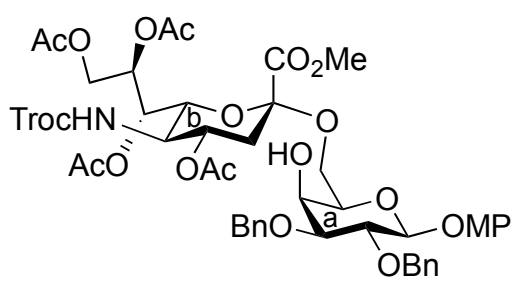

4-Methoxyphenyl (methyl 4,7,8,9-tetra-O-acetyl-3,5-dideoxy-5-(2,2,2-trichloroethoxycarbamoyl)-Dglycero- $\alpha$-D-galacto-2-nonulopyranosylonate)-(2 $\rightarrow 6)$-2,3-di-O-benzyl- $\beta$-D-galactopyranoside (6). To a mixture of $2(691 \mathrm{mg}, 0.964 \mathrm{mmol})$ and $5(300 \mathrm{mg}, 0.643 \mathrm{mmol})$ in $\mathrm{EtCN} / \mathrm{CH}_{2} \mathrm{Cl}_{2}(5: 1,9.6 \mathrm{~mL})$ was added $3 \AA$ molecular sieves $\left(991 \mathrm{mg}\right.$ ) at r.t. After stirring for $1 \mathrm{~h}$ and then cooling to $-30{ }^{\circ} \mathrm{C}$, NIS (324 mg, $1.44 \mathrm{mmol})$ and $\mathrm{TfOH}(12.7 \mu \mathrm{L}, 0.144 \mathrm{mmol})$ were added to the mixture. After stirring for $45 \mathrm{~min}$ at the same temperature as the reaction was monitored by TLC (1:3 EtOAc-toluene, twice development), the reaction was quenched by the addition of triethylamine. The precipitate was filtered through Celite. The filtrate was evaporated to remove EtCN and then diluted with $\mathrm{CHCl}_{3}$, washed with satd aq $\mathrm{Na}_{2} \mathrm{~S}_{2} \mathrm{O}_{3}$ and brine. The organic layer was subsequently dried over $\mathrm{Na}_{2} \mathrm{SO}_{4}$, concentrated and the residue was purified by silica gel column chromatography (1:5 EtOAc-toluene) to give 6 (473 mg, $69 \%$ ) along with its $\beta$-isomer (96 mg, 14\%). $[\alpha]_{\mathrm{D}}-13.9^{\circ}$ (c $\left.0.4, \mathrm{CHCl}_{3}\right) ;{ }^{1} \mathrm{H}_{-\mathrm{NMR}}\left(500 \mathrm{MHz}, \mathrm{CDCl}_{3}\right)$ $\delta 7.38-6.80(\mathrm{~m}, 14 \mathrm{H}, \mathrm{Ar}), 5.39(\mathrm{~m}, 1 \mathrm{H}, \mathrm{H}-8 \mathrm{~b}), 5.36$ (dd, $\left.1 \mathrm{H}, J_{6,7}=1.7 \mathrm{~Hz}, \mathrm{H}-7 \mathrm{~b}\right), 5.00(\mathrm{~d}, 1 \mathrm{H}$, $\left.J_{\text {gem }}=10.2 \mathrm{~Hz}, \mathrm{OCH}_{2}\right), 4.99(\mathrm{~m}, 1 \mathrm{H}, \mathrm{H}-4 \mathrm{~b}), 4.89\left(\mathrm{~d}, 1 \mathrm{H}, J_{\text {gem }}=12.2 \mathrm{~Hz}, \mathrm{OCH}_{2}\right), 4.86(\mathrm{~d}, 1 \mathrm{H}$, $\left.J_{5, \mathrm{NH}}=9.7 \mathrm{~Hz}, \mathrm{NH}\right), 4.84\left(\mathrm{~d}, 1 \mathrm{H}, J_{1,2}=8.0 \mathrm{~Hz}, \mathrm{H}-1 \mathrm{a}\right), 4.83\left(\mathrm{~d}, 1 \mathrm{H}, J_{\mathrm{gem}}=10.2 \mathrm{~Hz}, \mathrm{OCH}_{2}\right), 4.76(2 \mathrm{~d}$, $\left.2 \mathrm{H}, J_{\text {gem }}=12.0 \mathrm{~Hz}, \mathrm{OCH}_{2}\right), 4.47\left(\mathrm{~d}, 1 \mathrm{H}, J_{\mathrm{gem}}=12.2 \mathrm{~Hz}, \mathrm{OCH}_{2}\right), 4.32(\mathrm{dd}, 1 \mathrm{H}, \mathrm{H}-9 \mathrm{~b}), 4.19(\mathrm{dd}, 1 \mathrm{H}$, $J_{6,7}=1.7 \mathrm{~Hz}, \mathrm{H}-6 \mathrm{~b}$ ), 4.10 (dd, 1 H, H-9'b), 4.07 (near d, $1 \mathrm{H}, \mathrm{H}-4 \mathrm{a}$ ), 3.94-3.89 (m, 2 H, H-2a, H-5a), 3.81-3.75 (m, 7 H, H-6a, 2 OMe), 3.65-3.61 (m, 2 H, H-6'a, H-5b), 3.57 (dd, 1 H, H-3a), 2.67-2.63 (m, 2 H, H-3beq, OH), 2.12-1.99 (m, $12 \mathrm{H}, 4 \mathrm{Ac}), 1.91$ (t, $1 \mathrm{H}, \mathrm{H}-3 \mathrm{bax}) ;{ }^{13} \mathrm{C}-\mathrm{NMR}\left(100 \mathrm{MHz}, \mathrm{CDCl}_{3}\right)$ $\delta 193.2,191.4,170.7,170.3,170.1,169.9,167.9,155.2,154.0,151.7,138.4,137.9,129.0,128.4$, $128.3,128.2,128.1,127.8,127.7,127.6,125.3,118.6,114.4,102.9,98.7,95.3,80.6,78.7,77.2,75.3$, 74.5, 72.6, 72.3, 72.2, 68.6, 68.4, 67.5, 66.0, 63.1, 62.3, 55.6, 53.0, 51.6, 37.5, 29.7, 29.5, 21.4, 21.0, 20.8, 20.7. $\mathrm{m} / z$ (MALDI): found $[\mathrm{M}+\mathrm{Na}]^{+}$1094.32, $\mathrm{C}_{48} \mathrm{H}_{56} \mathrm{Cl}_{3} \mathrm{NO}_{20}$ calcd for $[\mathrm{M}+\mathrm{Na}]^{+} 1094.32$.

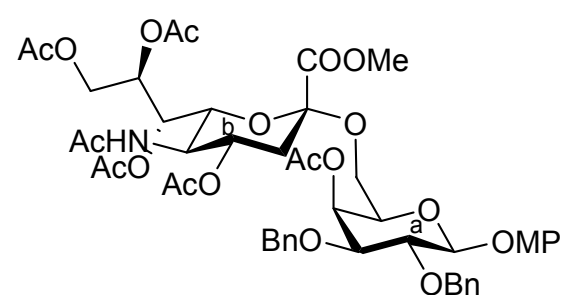

4-Methoxyphenyl (methyl 5-acetamido-4,7,8,9-tetra-O-acetyl-3,5-dideoxy-D-glycero- $\alpha$-D-galacto-2nonulopyranosylonate)-(2 $\rightarrow 6)-4-O$-acetyl-2,3-di-O-benzyl- $\beta$-D-galactopyranoside (8). To a solution of $6(3.77 \mathrm{~g}, 3.51 \mu \mathrm{mol})$ in $\mathrm{AcOH} / \mathrm{CH}_{2} \mathrm{Cl}_{2}(3: 2,70 \mathrm{~mL})$ was added $\mathrm{Zn} / \mathrm{Cu}$ couple $(18.9 \mathrm{~g})$ at r.t. The reaction mixture was heated to $40{ }^{\circ} \mathrm{C}$ and was stirred for $45 \mathrm{~min}$ at the same temperature as the reaction was monitored by TLC (4:1 toluene-EtOAc). The precipitate was filtered through Celite and the filtrate was co-evaporated with toluene. The obtained residue was exposed to high vacuum for $6 \mathrm{~h}$. The crude residue was dissolved in pyridine $(35 \mathrm{~mL})$ and acetic anhydride $(1.32 \mu \mathrm{L}, 14.0 \mathrm{mmol})$, DMAP $(4.3 \mathrm{mg}, 35.1 \mu \mathrm{mol})$ were then added to the mixture at $0{ }^{\circ} \mathrm{C}$. After stirring for $3 \mathrm{~d}$ at r.t as the reaction was monitored by TLC (2:1 toluene-EtOAc), the reaction mixture was evaporated. The 
residue was diluted with $\mathrm{CHCl}_{3}$, washed with $2 \mathrm{M} \mathrm{HCl}, \mathrm{H}_{2} \mathrm{O}$, satd aq $\mathrm{NaHCO}_{3}$ and brine, dried over $\mathrm{Na}_{2} \mathrm{SO}_{4}$, and concentrated. The obtained residue was purified by silica gel column chromatography (4:1 toluene-EtOAc) to give 8 (3.35 g, 97\%). [ $\alpha]_{\mathrm{D}}-11.3^{\circ}\left(\mathrm{c} 1.0, \mathrm{CHCl}_{3}\right) ;{ }^{1} \mathrm{H}-\mathrm{NMR}\left(500 \mathrm{MHz}, \mathrm{CDCl}_{3}\right)$ $\delta$ 7.37-7.24 (m, $10 \mathrm{H}, 2 \mathrm{Ph}), 6.97(2 \mathrm{~d}, 4 \mathrm{H}, \mathrm{Ar}), 5.95\left(\mathrm{~d}, 1 \mathrm{H}, J_{\mathrm{NH}, 5}=6.3 \mathrm{~Hz}, \mathrm{NH}\right), 5.62(\mathrm{~d}, 1 \mathrm{H}$, $\left.J_{3,4}=2.3 \mathrm{~Hz}, \mathrm{H}-4 \mathrm{a}\right), 5.41$ (m, $\left.1 \mathrm{H}, \mathrm{H}-8 \mathrm{~b}\right), 5.32$ (d, $\left.1 \mathrm{H}, \mathrm{H}-7 \mathrm{~b}\right), 4.96-4.81$ (m, $5 \mathrm{H}, \mathrm{H}-1 \mathrm{a}, \mathrm{H}-4 \mathrm{~b}$, $\left.3 \mathrm{OCH}_{2}\right), 4.68$ (d, $\left.1 \mathrm{H}, \mathrm{OCH}_{2}\right), 4.34$ (d, $\left.1 \mathrm{H}, \mathrm{H}-9 \mathrm{~b}\right), 4.14-4.05$ (m, $3 \mathrm{H}, \mathrm{H}-5 \mathrm{~b}, \mathrm{H}-6 \mathrm{~b}, \mathrm{H}-9 \mathrm{~b}$ ), 3.90-3.69 (m, 11 H, H-2a, H-3a, H-5a, H-6a, H-6'a, 2 OMe), 2.62 (m, 1 H, $J_{\text {gem }}=11.5$ Hz, H-3beq), 2.16-1.87 $(\mathrm{m}, 19 \mathrm{H}, 6 \mathrm{Ac}, \mathrm{H}-3 \mathrm{~b} a x) ;{ }^{13} \mathrm{C}-\mathrm{NMR}\left(100 \mathrm{MHz}, \mathrm{CDCl}_{3}\right) \delta 170.4,170.2,170.0,169.8,169.7,169.5$, $167.7,154.9,151.2,138.2,137.5,128.7,128.0,127.9,127.7,127.3,127.2,118.0,114.1,102.2,98.4$, 79.1, 78.3, 75.0, 72.4, 71.8, 71.3, 68.7, 68.3, 67.0, 65.9, 62.7, 62.3, 55.2, 52.5, 48.6, 37.5, 22.7, 20.7, 20.5, 20.4, 20.4, 20.3. $\mathrm{m} / \mathrm{z}$ (MALDI): found $[\mathrm{M}+\mathrm{Na}]^{+}$1004.17, $\mathrm{C}_{49} \mathrm{H}_{59} \mathrm{NO}_{20}$ calcd for $[\mathrm{M}+\mathrm{Na}]^{+} 1004.35$.

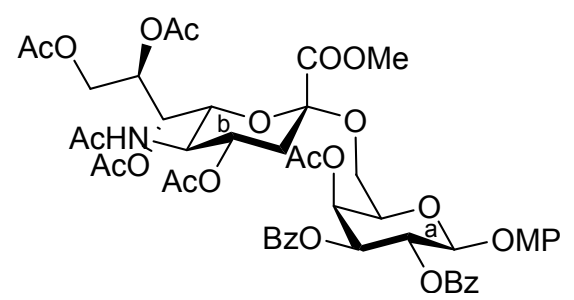

4-Methoxyphenyl (methyl 5-acetamido-4,7,8,9-tetra-O-acetyl-3,5-dideoxy-D-glycero- $\alpha$-D-galacto-2nonulopyranosylonate)-(2 $\rightarrow 6)-4-O$-acetyl-2,3-di-O-benzoyl- $\beta$-D-galactopyranoside (10). To a solution of $8(3.34 \mathrm{~g}, 3.41 \mu \mathrm{mol})$ in 1,4-dioxane $(34 \mathrm{~mL})$ was added $\mathrm{Pd}(\mathrm{OH})_{2} / \mathrm{C}(3.34 \mathrm{~g})$. After stirring for $45 \mathrm{~min}$ at r.t. under a hydrogen atmosphere as the reaction was monitored by TLC $\left(15: 1 \mathrm{CHCl}_{3}-\mathrm{MeOH}\right)$, the mixture was filtered through Celite. The filtrate was concentrated and the obtained crude residue was roughly purified by silica gel column chromatography. The obtained product was exposed to high vacuum for $24 \mathrm{~h}$. The residue was then dissolved in pyridine $(34 \mathrm{~mL})$. Benzoic anhydride $(3.09 \mathrm{~g}$, $13.6 \mathrm{mmol})$ and DMAP $(20.8 \mathrm{mg}, 0.171 \mu \mathrm{mol})$ were added to the mixture at $0{ }^{\circ} \mathrm{C}$. After stirring for $9 \mathrm{~h}$ at r.t. as the reaction was monitored by TLC $\left(15: 1 \mathrm{CHCl}_{3}-\mathrm{MeOH}\right)$, the reaction was quenched by the addition of $\mathrm{MeOH}$ at $0{ }^{\circ} \mathrm{C}$. The mixture was co-evaporated with toluene and the residue was then diluted with $\mathrm{CHCl}_{3}$, and washed with $2 \mathrm{M} \mathrm{HCl}, \mathrm{H}_{2} \mathrm{O}$, satd aq $\mathrm{NaHCO}_{3}$ and brine. The organic layer was subsequently dried over $\mathrm{Na}_{2} \mathrm{SO}_{4}$, and concentrated. The resulting residue was purified by silica gel column chromatography $\left(1: 1\right.$ toluene-EtOAc) to give $\mathbf{1 0}(3.22 \mathrm{~g}, 94 \%)$. $[\alpha]_{\mathrm{D}}+40.3^{\circ}\left(\mathrm{c} 1.0, \mathrm{CHCl}_{3}\right)$; ${ }^{1} \mathrm{H}-\mathrm{NMR}\left(500 \mathrm{MHz}, \mathrm{CDCl}_{3}\right) \delta$ 8.00-7.37 (m, $\left.10 \mathrm{H}, 2 \mathrm{Ph}\right), 7.06-6.76(2 \mathrm{~d}, \mathrm{Ar}), 5.91$ (near t, $1 \mathrm{H}$, $\left.J_{1,2}=7.8 \mathrm{~Hz}, J_{2,3}=10.6 \mathrm{~Hz}, \mathrm{H}-2 \mathrm{a}\right), 5.78\left(\mathrm{~d}, 1 \mathrm{H}, J_{3,4}=3.4 \mathrm{~Hz}, \mathrm{H}-4 \mathrm{a}\right), 5.60$ (dd, $\left.1 \mathrm{H}, \mathrm{H}-3 \mathrm{a}\right), 5.50$ (m, $\left.1 \mathrm{H}, J_{7,8}=6.8 \mathrm{~Hz}, \mathrm{H}-8 \mathrm{~b}\right), 5.33-5.27$ (m, $\left.2 \mathrm{H}, \mathrm{H}-1 \mathrm{a}, \mathrm{H}-7 \mathrm{~b}\right), 5.15(\mathrm{~d}, 1 \mathrm{H}, \mathrm{NH}), 4.87$ (m, $1 \mathrm{H}, J_{3 \mathrm{eq}, 4}=4.6 \mathrm{~Hz}$, $\mathrm{H}-4 \mathrm{~b}), 4.41$ (dd, $\left.1 \mathrm{H}, J_{\text {gem }}=12.4 \mathrm{~Hz}, \mathrm{H}-9\right), 4.30$ (near t, $\left.1 \mathrm{H}, J_{5,6}=6.9 \mathrm{~Hz}, \mathrm{H}-5 \mathrm{a}\right), 4.17-4.02(\mathrm{~m}, 3 \mathrm{H}$, H-5b, H-6b, H-9'b), 3.89-3.73 (m, 7 H, 2 OMe, H-6a), 3.58 (near t, 1 H, $J_{\text {gem }}=10.1$ Hz, H-6'a), 2.55 $\left(\mathrm{dd}, 1 \mathrm{H}, J_{\text {gem }}=12.4 \mathrm{~Hz}, \mathrm{H}-3 \mathrm{beq}\right), 2.25-1.89$ (m, $\left.19 \mathrm{H}, 6 \mathrm{Ac}, \mathrm{H}-3 \mathrm{~b} a x\right) ;{ }^{13} \mathrm{C}-\mathrm{NMR}\left(100 \mathrm{MHz}, \mathrm{CDCl}_{3}\right) \delta$ $170.5,170.4$, 170.0, 169.7, 169.5, 167.7, 165.1, 165.0, 155.1, 151.0, 133.0, 129.4, 129.3, 129.1, 128.8, 128.1, 128.1, 118.2, 114.1, 100.1, 98.9, 72.5, 71.7, 71.6, 69.4, 68.6, 67.9, 67.0, 62.9, 55.2, 52.7, 48.7, 37.6, 22.8, 20.8, 20.5, 20.4, 20.3. $\mathrm{m} / \mathrm{z}$ (MALDI): found $[\mathrm{M}+\mathrm{Na}]^{+} 1032.21, \mathrm{C}_{49} \mathrm{H}_{55} \mathrm{NO}_{22}$ calcd for $[\mathrm{M}+\mathrm{Na}]^{+} 1032.31$. 


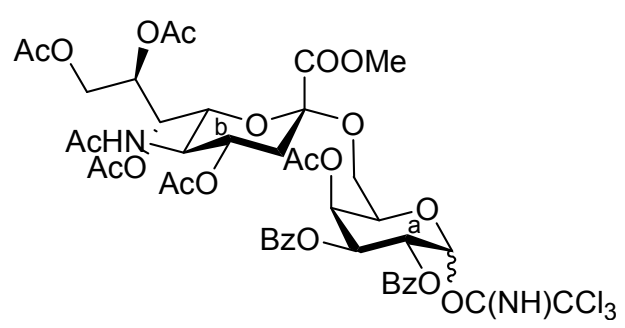

(Methyl 5-acetamido-4,7,8,9-tetra-O-acetyl-3,5-dideoxy-D-glycero- $\alpha$-D-galacto-2-nonulopyranosylonate)$(2 \rightarrow 6)-4-O$-acetyl-2,3-di-O-benzoyl-D-galactopyranosyl trichloroacetimidate (12). To a solution of 10 (3.22 g, $3.19 \mathrm{mmol})$ in $\mathrm{MeCN} / \mathrm{PhMe} / \mathrm{H}_{2} \mathrm{O}(32 \mathrm{~mL}, 6: 5: 3)$ was added diammonium cerium (IV) nitrate $(\mathrm{CAN} ; 17.5 \mathrm{~g}, 31.9 \mathrm{mmol})$ at r.t. The mixture was stirred for $2 \mathrm{~h}$ at r.t., as the proceeding of the reaction was monitored by TLC $\left(10: 1 \mathrm{CHCl}_{3}-\mathrm{MeOH}\right)$. The reaction mixture was diluted with $\mathrm{CHCl}_{3}$ and washed with $\mathrm{H}_{2} \mathrm{O}$, satd aq $\mathrm{NaHCO}_{3}$ and brine. The organic layer was subsequently dried over $\mathrm{Na}_{2} \mathrm{SO}_{4}$ and concentrated. The resulting residue was purified by silica gel column chromatography (70:1 $\left.\mathrm{CHCl}_{3}-\mathrm{MeOH}\right)$ to give 11. The obtained hemiacetal compound 11 (2.27 g, $\left.2.51 \mathrm{mmol}\right)$ was dissolved in $\mathrm{CH}_{2} \mathrm{Cl}_{2}(50 \mathrm{~mL})$. To the mixture was added $\mathrm{CCl}_{3} \mathrm{CN}(2.5 \mathrm{~mL}, 25.1 \mathrm{mmol})$, DBU $(449 \mu \mathrm{L}$, $3.01 \mathrm{mmol})$ at $0{ }^{\circ} \mathrm{C}$. After stirring for $6 \mathrm{~h}$ at $0{ }^{\circ} \mathrm{C}$ as the reaction was monitored by TLC (15:1 $\mathrm{CHCl}_{3}-\mathrm{MeOH}$ ), the reaction mixture was evaporated. The crude residue was purified by silica gel column chromatography $\left(70: 1 \mathrm{CHCl}_{3}-\mathrm{MeOH}\right)$ to give 12 (2.10 g, 63\% over 2 steps). 12 $\alpha$ : ${ }^{1} \mathrm{H}-\mathrm{NMR}$ $\left(500 \mathrm{MHz}, \mathrm{CDCl}_{3}\right) \delta 8.61(\mathrm{~s}, 1 \mathrm{H}, \mathrm{C}=\mathrm{NH}), 7.95-7.28(\mathrm{~m}, 10 \mathrm{H}, 2 \mathrm{Ph}), 6.80\left(\mathrm{~d}, 1 \mathrm{H}, J_{1,2}=3.4 \mathrm{~Hz}\right.$, H-1a), 5.90-5.82 (m, 2 H, H-2a, H-3a), 5.79 (m, 1 H, J3,4 $=2.8$ Hz, H-4a), 5.38-5.30 (m, 3 H, H-7b, $\mathrm{H}-8 \mathrm{~b}, \mathrm{NHb}), 4.87-4.86$ (m, $1 \mathrm{H}, \mathrm{H}-4 \mathrm{~b}), 4.51$ (br t, $1 \mathrm{H}, \mathrm{H}-5 \mathrm{a}), 4.28$ (dd, $1 \mathrm{H}, J_{\text {gem }}=12.6 \mathrm{~Hz}, J_{8,9}=2.9 \mathrm{~Hz}$, H-9b), 4.11 (dd, 1 H, J8, , $^{\prime}=5.9$ Hz, H-9'b), 4.06-3.97 (m, 3 H, H-6a, H-5b, H-6b), 3.78 (s, 3 H, COOMe), $3.42\left(\mathrm{dd}, 1 \mathrm{H}, J_{5,6^{\prime}}=2.6 \mathrm{~Hz}, J_{\text {gem }}=10.6 \mathrm{~Hz}, \mathrm{H}-6 \mathrm{\prime}\right.$ ) $), 3.55\left(\mathrm{dd}, 1 \mathrm{H}, J_{\text {gem }}=11.6 \mathrm{~Hz}, J_{3 \mathrm{eq}, 4}=3.6 \mathrm{~Hz}\right.$, H-3beq), 2.35-1.87 (m, 19 H, 6 Ac, H-3bax).

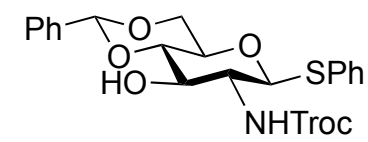

Phenyl 4,6-O-benzylidene-2-deoxy-1-thio-2-(2,2,2-trichloroethoxycarbamoyl)- $\beta$-D-glucopyranoside (14). To a solution of $13(8.43 \mathrm{~g}, 14.7 \mathrm{mmol})$ in $\mathrm{MeOH}(84 \mathrm{~mL})$ was added $\mathrm{NaOMe}(28 \%$ solution in $\mathrm{MeOH}, 39.7 \mathrm{mg}, 0.735 \mathrm{mmol})$ at $0{ }^{\circ} \mathrm{C}$. After stirring for $2.5 \mathrm{~h}$ at room temperature as the reaction was monitored by TLC $\left(10: 1 \mathrm{CHCl}_{3}-\mathrm{MeOH}\right)$, the reaction was neutralized with Dowex $\left(\mathrm{H}^{+}\right)$resin. The resin was filtered through cotton and the filtrate was then evaporated. The residue was exposed to high vacuum for $12 \mathrm{~h}$. The obtained crude mixture was then dissolved in THF/MeCN (1:4, $135 \mathrm{~mL})$. To the mixture were added benzaldehyde dimethyl acetal $(4.39 \mathrm{~mL}, 29.4 \mathrm{mmol})$ and $( \pm)$-camphor-10-sulfonic acid (CSA) $(512 \mathrm{mg}, 2.21 \mathrm{mmol})$ at $0{ }^{\circ} \mathrm{C}$. After stirring for $1 \mathrm{~h}$ at room temperature as the reaction was monitored by TLC $\left(20: 1 \mathrm{CHCl}_{3}-\mathrm{MeOH}\right)$, the reaction was quenched by the addition of triethylamine. The reaction mixture was concentrated and then subjected to crystallization from hot acetone $/ n$-hexane to give $14(6.27 \mathrm{~g}, 80 \%)$ as a white crystal. The physical data of $\mathbf{1 4}$ was identical to those reported in the literature [27]. 


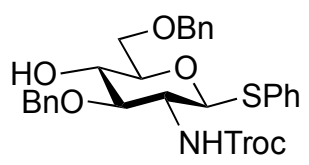

Phenyl 3,6-di-O-benzyl-2-deoxy-1-thio-2-(2,2,2-trichloroethoxycarbamoyl)- $\beta$-D-glucopyranoside (15). To a solution of $14(4.89 \mathrm{~g}, 9.15 \mathrm{mmol})$ in THF/toluene $(1: 4,9.0 \mathrm{~mL})$ was added TESOTf $(4.1 \mathrm{~mL}$, $18.3 \mathrm{mmol}$ ) at $-20{ }^{\circ} \mathrm{C}$. After stirring for $45 \mathrm{~min}$ at $-20{ }^{\circ} \mathrm{C}$, benzaldehyde $(4.7 \mathrm{~mL}, 45.7 \mathrm{mmol})$ and triethylsilane $(2.2 \mathrm{~mL}, 13.7 \mathrm{mmol})$ were added to the mixture. After stirring for $2 \mathrm{~h}$ at $-20{ }^{\circ} \mathrm{C}$ as the reaction was monitored by TLC (1:4 EtOAc-toluene), the reaction was quenched by satd aq $\mathrm{Na}_{2} \mathrm{CO}_{3}$. Dilution of the mixture with EtOAc provided a solution, which was then washed with satd aq $\mathrm{Na}_{2} \mathrm{CO}_{3}$ and brine. The organic layer was subsequently dried over $\mathrm{Na}_{2} \mathrm{SO}_{4}$ and concentrated. The obtained residue was exposed to high vacuum for $24 \mathrm{~h}$. The resulting residue was dissolved in $\mathrm{CH}_{2} \mathrm{Cl}_{2}(183 \mathrm{~mL})$ and cooled to $0{ }^{\circ} \mathrm{C} . \mathrm{BF}_{3} \cdot \mathrm{OEt}_{2}(4.7 \mathrm{~mL}, 18.3 \mathrm{mmol})$ and triethylsilane $\left(14.6 \mathrm{~mL}, 91.5 \mathrm{mmol}^{2}\right.$ were added to the solution at $0{ }^{\circ} \mathrm{C}$ and the mixture was then stirred for $1 \mathrm{~h}$ at $0{ }^{\circ} \mathrm{C}$ as the reaction was monitored by TLC (1:4 EtOAc-toluene). The reaction was quenched by the addition of satd aq $\mathrm{Na}_{2} \mathrm{CO}_{3}$ at $0{ }^{\circ} \mathrm{C}$ and then diluted with $\mathrm{CHCl}_{3}$, and washed with satd aq $\mathrm{Na}_{2} \mathrm{CO}_{3}$ and brine. The organic layer was subsequently dried over $\mathrm{Na}_{2} \mathrm{SO}_{4}$ and concentrated. The resulting residue was purified by silica gel column chromatography $\left(200: 1 \mathrm{CHCl}_{3}-\mathrm{MeOH}\right)$ to give $15(4.50 \mathrm{~g}, 78 \%)$. [ $\left.\alpha\right]_{\mathrm{D}}-24.2^{\circ}$ (c 0.3 , $\left.\mathrm{CHCl}_{3}\right) ;{ }^{1} \mathrm{H}-\mathrm{NMR}\left(400 \mathrm{MHz}, \mathrm{CDCl}_{3}\right) \delta 7.49-7.21(\mathrm{~m}, 15 \mathrm{H}, 3 \mathrm{Ph}), 5.17\left(\mathrm{~d}, 1 \mathrm{H}, J_{2, \mathrm{NH}}=8.2 \mathrm{~Hz}, \mathrm{NH}\right)$, $4.91\left(\mathrm{~d}, 1 \mathrm{H}, J_{1,2}=10.1 \mathrm{~Hz}, \mathrm{H}-1\right), 4.75\left(\mathrm{~s}, 4 \mathrm{H}, 2 \mathrm{OCH}_{2}\right), 4.56\left(2 \mathrm{~d}, 2 \mathrm{H}, \mathrm{OCH}_{2}\right), 3.77-3.67(\mathrm{~m}, 4 \mathrm{H}$, H-3, H-5, H-6, H-6'), 3.52-3.42 (m, 2 H, H-2, H-4), 2.85 (s, $1 \mathrm{H}, \mathrm{OH}) ;{ }^{13} \mathrm{C}-\mathrm{NMR}\left(100 \mathrm{MHz}, \mathrm{CDCl}_{3}\right) \delta$ $153.8,137.9,137.7,132.7,132.3,128.9,128.5,128.4,128.1,128.0,127.8,127.7,95.4,86.0,81.9$, 78.0, 74.5, 74.4, 73.7, 72.6, 70.4, 56.0. $\mathrm{m} / z$ (MALDI): found $[\mathrm{M}+\mathrm{Na}]^{+} 648.05, \mathrm{C}_{29} \mathrm{H}_{30} \mathrm{Cl}_{3} \mathrm{NO}_{6} \mathrm{~S}$ calcd for $[\mathrm{M}+\mathrm{Na}]^{+} 648.08$.

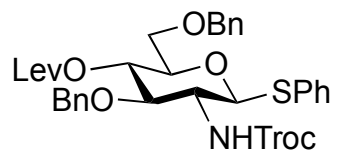

Phenyl 3,6-di-O-benzyl -2-deoxy-4-O-levulinoyl-1-thio-2-(2,2,2-trichloroethoxycarbamoyl)- $\beta$-Dglucopyranoside (16). To a solution of $15(200 \mathrm{mg}, 0.314 \mathrm{mmol})$ in $\mathrm{CH}_{2} \mathrm{Cl}_{2}(3.1 \mathrm{~mL})$ were added levulinic acid (55 mg, $0.472 \mathrm{mmol}), \mathrm{EDC} \cdot \mathrm{HCl}(90 \mathrm{mg}, 0.472 \mathrm{mmol})$, and DMAP (4.2 $\mathrm{mg}, 3.44 \mu \mathrm{mol})$. After stirring for $4 \mathrm{~h}$ at r.t. as the reaction was monitored by TLC (2:3 EtOAc- $n$-hexane), the reaction mixture was diluted with $\mathrm{CHCl}_{3}$, and washed with $2 \mathrm{M} \mathrm{HCl}$ and brine. The organic layer was subsequently dried over $\mathrm{Na}_{2} \mathrm{SO}_{4}$, and concentrated, and the obtained residue was purified by silica gel column chromatography (1:4 EtOAc- $n$-hexane) to give $16(215 \mathrm{mg}, 93 \%)$. $[\alpha]_{\mathrm{D}}+3.7^{\circ}\left(\mathrm{c} 0.9, \mathrm{CHCl}_{3}\right)$; ${ }^{1} \mathrm{H}-\mathrm{NMR}\left(600 \mathrm{MHz}\right.$, acetone- $\left.d_{6}\right) \delta 7.53-7.24 \mathrm{~m}, 16 \mathrm{H}, 3 \mathrm{Ph}, \mathrm{NH} .5 .06\left(\mathrm{~d}, 1 \mathrm{H}, J_{1,2}=10.3 \mathrm{~Hz}, \mathrm{H}-1\right)$, $5.03\left(\mathrm{t}, 1 \mathrm{H}, J_{3,4}=9.6 \mathrm{~Hz}, J_{4,5}=9.7 \mathrm{~Hz}, \mathrm{H}-4\right), 4.83\left(2 \mathrm{~d}, 2 \mathrm{H}, J_{\mathrm{gem}}=12.4 \mathrm{~Hz}, \mathrm{OCH}_{2}\right), 4.72(2 \mathrm{~d}, 2 \mathrm{H}$, $\left.J_{\mathrm{gem}}=11.0 \mathrm{~Hz}, \mathrm{OCH}_{2}\right), 4.52\left(2 \mathrm{~d}, 2 \mathrm{H}, J_{\mathrm{gem}}=11.7 \mathrm{~Hz}, \mathrm{OCH}_{2}\right), 3.99\left(\mathrm{t}, 1 \mathrm{H}, J_{2,3}=9.7 \mathrm{~Hz}, \mathrm{H}-3\right), 3.79-3.75$ (m, $2 \mathrm{H}, \mathrm{H}-2, \mathrm{H}-5), 3.65$ (dd, $\left.1 \mathrm{H}, J_{\text {gem }}=11.0 \mathrm{~Hz}, J_{5,6}=2.8 \mathrm{~Hz}, \mathrm{H}-6\right), 3.65$ (dd, $1 \mathrm{H}, J_{5,6^{\prime}}=6.9 \mathrm{~Hz}, \mathrm{H}-6^{\prime}$ ), 2.73-2.40 (m, $\left.4 \mathrm{H}, \mathrm{CH}_{2} \mathrm{CH}_{2} \mathrm{C}(=\mathrm{O}) \mathrm{O}\right), 2.09$ (s, $\left.3 \mathrm{H}, \mathrm{C}(=\mathrm{O}) \mathrm{CH}_{3}\right) ;{ }^{13} \mathrm{C}-\mathrm{NMR}\left(150 \mathrm{MHz}, \mathrm{CDCl}_{3}\right) \delta 166.1$, $153.7,137.6,137.5,132.6,132.1,129.0,128.5,128.3,128.0,128.0,127.9,127.7,95.4,85.2,79.0$, 76.8, 74.5, 74.4, 73.6, 73.2, 69.5, 56.7, 40.5. $\mathrm{m} / z$ (MALDI): found $[\mathrm{M}+\mathrm{Na}]^{+} 746.09, \mathrm{C}_{34} \mathrm{H}_{36} \mathrm{Cl}_{3} \mathrm{NO}_{8} \mathrm{~S}$ calcd for $[\mathrm{M}+\mathrm{Na}]^{+} 746.11$. 


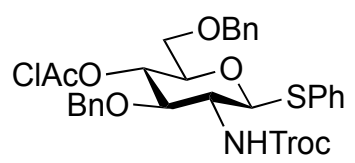

Phenyl 3,6-di-O-benzyl-4-O-chloroacetyl-2-deoxy-1-thio-2-(2,2,2-trichloroethoxycarbamoyl)- $\beta$ D-glucopyranoside (17). To a solution of $15(200 \mathrm{mg}, 0.314 \mathrm{mmol})$ in THF $(3.1 \mathrm{~mL})$ were added monochloroacetic anhydride $(81 \mathrm{mg}, 0.472 \mathrm{mmol})$ and DMAP $(0.5 \mathrm{mg}, 4.09 \mu \mathrm{mol})$ at $0{ }^{\circ} \mathrm{C}$. After stirring for $7 \mathrm{~h}$ at r.t. as the reaction was monitored by TLC (2:3 EtOAc- $n$-hexane), THF was evaporated and the residue was then diluted with $\mathrm{CHCl}_{3}$, and washed with $2 \mathrm{M} \mathrm{HCl}, \mathrm{H}_{2} \mathrm{O}$, satd aq $\mathrm{NaHCO}_{3}$, and brine. The organic layer was subsequently dried over $\mathrm{Na}_{2} \mathrm{SO}_{4}$, and concentrated. The residue was purified by silica gel column chromatography (1:8 EtOAc- $n$-hexane) to give $\mathbf{1 7}$ (200 mg, 90\%). $[\alpha]_{\mathrm{D}}-2.6^{\circ}$ (c 1.0, $\left.\mathrm{CHCl}_{3}\right) ;{ }^{1} \mathrm{H}-\mathrm{NMR}\left(600 \mathrm{MHz}, \mathrm{CDCl}_{3}\right) \delta 7.51-7.21(\mathrm{~m}, 15 \mathrm{H}, 3 \mathrm{Ph}), 5.37(\mathrm{~d}, 1 \mathrm{H}$, $\left.J_{\mathrm{NH}, 2}=8.2 \mathrm{~Hz}, \mathrm{NH}\right), 5.12\left(\mathrm{~d}, 1 \mathrm{H}, J_{1,2}=10.3 \mathrm{~Hz}, \mathrm{H}-1\right), 5.07$ (near t, $1 \mathrm{H}, J_{3,4}=8.9 \mathrm{~Hz}, J_{4,5}=9.6 \mathrm{~Hz}$, $\mathrm{H}-4), 4.75\left(2 \mathrm{~d}, 2 \mathrm{H}, J_{\text {gem }}=11.8 \mathrm{~Hz}, \mathrm{OCH}_{2}\right), 4.61\left(2 \mathrm{~d}, 2 \mathrm{H}, J_{\text {gem }}=11.7 \mathrm{~Hz}, \mathrm{OCH}_{2}\right), 4.48(\mathrm{~m}, 2 \mathrm{H}$, $J_{\text {gem }}=11.7 \mathrm{~Hz}, \mathrm{OCH}_{2}$ ), 4.11 (near t, $\left.1 \mathrm{H}, J_{2,3}=9.6 \mathrm{~Hz}, J_{3,4}=8.9 \mathrm{~Hz}, \mathrm{H}-3\right), 3.68-3.55(\mathrm{~m}, 5 \mathrm{H}, \mathrm{H}-5, \mathrm{H}-6$, H-6', $\mathrm{CH}_{2} \mathrm{Cl}$ ), 3.36 (br dd, $\left.1 \mathrm{H}, J_{2,3}=9.6 \mathrm{~Hz}, \mathrm{H}-2\right) ;{ }^{13} \mathrm{C}-\mathrm{NMR}\left(125 \mathrm{MHz}, \mathrm{CDCl}_{3}\right) \delta 166.1,153.7$, 137.7, 137.6, 132.8, 132.0, 129.1, 128.5, 128.4, 128.2, 128.0, 127.9, 127.8, 95.4, 85.2, 79.0, 77.6, 74.7, 74.5, 73.7, 73.4, 69.7, 56.9, 40.5. $\mathrm{m} / z$ (MALDI): found $[\mathrm{M}+\mathrm{Na}]^{+} 724.05, \mathrm{C}_{31} \mathrm{H}_{31} \mathrm{Cl}_{4} \mathrm{NO}_{7} \mathrm{~S}$ calcd for $[\mathrm{M}+\mathrm{Na}]^{+} 724.05$.

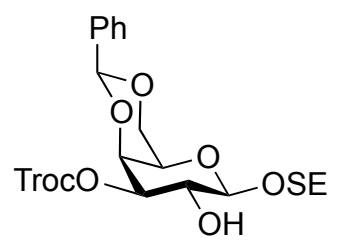

2-(Trimethylsilyl)ethyl 4,6-O-benzylidene-3-O-(2,2,2-trichloroethoxycarbonyl)- $\beta$-D-galactopyranoside (19). To a solution of $18(49.5 \mathrm{mg}, 0.134 \mathrm{mmol})$ in pyridine $/ \mathrm{CH}_{2} \mathrm{Cl}_{2}(1: 4,1.3 \mathrm{~mL})$ was added trichloroethyl chloroformate $(20 \mu \mathrm{L}, 0.148 \mathrm{mmol})$ at $-40{ }^{\circ} \mathrm{C}$. After stirring for $3 \mathrm{~h}$ at the same temperature as the reaction was monitored by TLC $\left(30: 1 \mathrm{CHCl}_{3}-\mathrm{MeOH}\right)$, solvents were removed by co-evaporation with toluene, and then the residue was diluted with $\mathrm{CHCl}_{3}$, washed with $2 \mathrm{M} \mathrm{HCl}, \mathrm{H}_{2} \mathrm{O}$, satd aq $\mathrm{NaHCO}_{3}$, and brine. The organic layer was subsequently dried over $\mathrm{Na}_{2} \mathrm{SO}_{4}$, and concentrated. The resulting residue was purified by silica gel column chromatography (1:5 EtOAc- $n$-hexane) to give 19 (59.1 mg, 81\%). $[\alpha]_{\mathrm{D}}+41.7^{\circ}\left(\mathrm{c} 1.0, \mathrm{CHCl}_{3}\right) ;{ }^{1} \mathrm{H}-\mathrm{NMR}\left(400 \mathrm{MHz}, \mathrm{CDCl}_{3}\right) \delta 7.48-7.30(\mathrm{~m}, 5 \mathrm{H}, \mathrm{Ph})$, 5.49 (s, $1 \mathrm{H}, \mathrm{PhCH}<), 4.82-4.72\left(\mathrm{~m}, 3 \mathrm{H}, \mathrm{H}-3, \mathrm{OCH}_{2} \mathrm{CCl}_{3}\right), 4.45$ (d, $\left.1 \mathrm{H}, J_{3,4}=3.7 \mathrm{~Hz}, \mathrm{H}-4\right), 4.34$ $\left(\mathrm{d}, 1 \mathrm{H}, J_{1,2}=7.7 \mathrm{~Hz}, \mathrm{H}-1\right), 4.33\left(\mathrm{~d}, 1 \mathrm{H}, J_{\text {gem }}=13.8 \mathrm{~Hz}, \mathrm{H}-6\right), 4.08-4.01$ (m, $3 \mathrm{H}, \mathrm{H}-2, \mathrm{H}-6$ ', $\mathrm{OCH}_{2} \mathrm{CH}_{2} \mathrm{SiMe}_{3}$ ), 3.60-3.53 (m, $\left.1 \mathrm{H}, \mathrm{OCH}_{2} \mathrm{CH}_{2} \mathrm{SiMe}_{3}\right), 3.48$ (s, $\left.1 \mathrm{H}, \mathrm{H}-5\right), 2.51$ (br s, $1 \mathrm{H}, \mathrm{OH}$ ), 1.03-0.97 (m, $\left.2 \mathrm{H}, \mathrm{OCH}_{2} \mathrm{CH}_{2} \mathrm{SiMe}_{3}\right), 0.00\left(\mathrm{~s}, 9 \mathrm{H}, \mathrm{OCH}_{2} \mathrm{CH}_{2} \mathrm{SiMe}_{3}\right) ;{ }^{13} \mathrm{C}-\mathrm{NMR}\left(100 \mathrm{MHz}, \mathrm{CDCl}_{3}\right) \delta$ 153.6, 137.3, 129.0, 128.1, 126.2, 102.3, 100.9, 94.2, 78.2, 77.2, 76.9, 73.0, 69.0, 68.5, 67.5, 66.2, 18.1, -1.4. $\mathrm{m} / z$ (MALDI): found $[\mathrm{M}+\mathrm{Na}]^{+}$565.11, $\mathrm{C}_{16} \mathrm{H}_{16} \mathrm{Cl}_{3} \mathrm{O}_{7}$ calcd for $[\mathrm{M}+\mathrm{Na}]^{+}$565.06. 


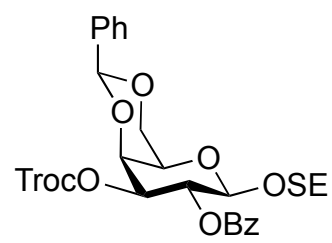

2-(Trimethylsilyl)ethyl 2-O-benzoyl-4,6-O-benzylidene-3-O-(2,2,2-trichloroethoxycarbonyl)- $\beta$ - $D$ galactopyranoside (20). To a solution of 19 (2.39 g, $4.39 \mathrm{mmol})$ in THF (1.3 mL) were added benzoic anhydride $(1.49 \mathrm{~g}, 6.59 \mathrm{mmol})$ and DMAP $(268 \mathrm{mg}, 2.20 \mu \mathrm{mol})$ at $0{ }^{\circ} \mathrm{C}$. After stirring for $8.5 \mathrm{~h}$ at r.t. as the reaction was monitored by TLC $\left(30: 1 \mathrm{CHCl}_{3}-\mathrm{MeOH}\right)$, THF was evaporated and the residue was then diluted with $\mathrm{CHCl}_{3}$, washed with $2 \mathrm{M} \mathrm{HCl}, \mathrm{H}_{2} \mathrm{O}$, satd aq $\mathrm{NaHCO}_{3}$, and brine. The organic layer was subsequently dried over $\mathrm{Na}_{2} \mathrm{SO}_{4}$, concentrated. The residue was purified by silica gel column chromatography (20:1 toluene-EtOAc) to give 20 (2.90 g, quant.). $[\alpha]_{\mathrm{D}}+38.8^{\circ}\left(\mathrm{c} 1.2, \mathrm{CHCl}_{3}\right) ;{ }^{1} \mathrm{H}-\mathrm{NMR}$ $\left(400 \mathrm{MHz} \mathrm{CDCl}_{3}\right) \delta 8.11-7.43(\mathrm{~m}, 10 \mathrm{H}, 2 \mathrm{Ph}), 5.79-7.75$ (near t, $1 \mathrm{H}, J_{2,3}=10.6 \mathrm{~Hz}, J_{1,2}=7.8 \mathrm{~Hz}$, $\mathrm{H}-2), 5.64$ (s, $1 \mathrm{H}, \mathrm{PhCH}<$ ), 5.22 (dd, $\left.1 \mathrm{H}, J_{3,4}=3.7 \mathrm{~Hz}, \mathrm{H}-3\right), 4.81-4.78\left(2 \mathrm{~d}, 2 \mathrm{H}, \mathrm{OCH}_{2} \mathrm{CCl}_{3}, \mathrm{H}-1\right)$, $4.68\left(\mathrm{~d}, 1 \mathrm{H}, J_{\text {gem }}=11.9 \mathrm{~Hz}, \mathrm{OCH}_{2} \mathrm{CCl}_{3}\right), 4.58(\mathrm{~d}, 1 \mathrm{H}, \mathrm{H}-4), 4.67-4.50\left(\mathrm{dd}, 1 \mathrm{H}, J_{\text {gem }}=12.4 \mathrm{~Hz}\right.$, $\left.J_{5,6}=1.8 \mathrm{~Hz}, \mathrm{H}-6\right), 4.23-4.19\left(\mathrm{dd}, 1 \mathrm{H}, J_{5,6^{\prime}}=1.8 \mathrm{~Hz}, \mathrm{H}-6^{\prime}\right), 4.15-4.08\left(\mathrm{~m}, 1 \mathrm{H}, \mathrm{OCH}_{2} \mathrm{CH}_{2} \mathrm{SiMe}_{3}\right)$, 3.68-3.62 (m, $2 \mathrm{H}, \mathrm{H}-5, \mathrm{OCH}_{2} \mathrm{CH}_{2} \mathrm{SiMe}_{3}$ ), 1.03-0.91 (m, $\left.2 \mathrm{H}, \mathrm{OCH}_{2} \mathrm{CH}_{2} \mathrm{SiMe}_{3}\right), 0.00(\mathrm{~s}, 9 \mathrm{H}$, $\mathrm{OCH}_{2} \mathrm{CH}_{2} \mathrm{SiMe}$ ); ${ }^{13} \mathrm{C}-\mathrm{NMR}\left(100 \mathrm{MHz}, \mathrm{CDCl}_{3}\right) \delta 164.8,153.7,137.2,133.1,129.8,129.7,129.1$, $128.3,128.2,126.4,101.1,100.5,94.1,76.8,76.6,73.4,69.2,68.9,67.0,66.2,17.9,-1.5 . \mathrm{m} / \mathrm{z}$ (MALDI): found $[\mathrm{M}+\mathrm{Na}]^{+} 669.09, \mathrm{C}_{28} \mathrm{H}_{33} \mathrm{Cl}_{3} \mathrm{O}_{9} \mathrm{Si}$ calcd for $[\mathrm{M}+\mathrm{Na}]^{+} 669.09$.

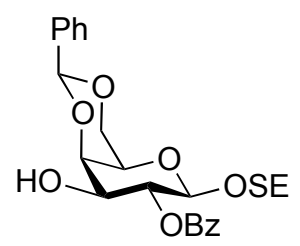

2-(Trimethylsilyl)ethyl 2-O-benzoyl-4,6-O-benzylidene- $\beta$-D-galactopyranoside (21). To a solution of $20(2.18 \mathrm{~g}, 3.36 \mu \mathrm{mol})$ in $\mathrm{AcOH} / \mathrm{CH}_{2} \mathrm{Cl}_{2}(3: 2,33.6 \mathrm{~mL})$ was added $\mathrm{Zn} / \mathrm{Cu}$ couple $(6.00 \mathrm{~g})$ at r.t. The reaction mixture was stirred for $1 \mathrm{~h}$ at r.t. as the reaction was monitored by TLC $\left(30: 1 \mathrm{CHCl}_{3}-\mathrm{MeOH}\right)$. The precipitate was filtered through Celite and the filtrate was co-evaporated with toluene. The residue was diluted with $\mathrm{CHCl}_{3}$ and washed with satd aq $\mathrm{NaHCO}_{3}$ and brine, dried over $\mathrm{Na}_{2} \mathrm{SO}_{4}$, and concentrated. The obtained residue was purified by silica gel column chromatography (4:1 EtOAc- $n$-hexane) to give 21 (1.33 g, 84\%). [ $\alpha]_{\mathrm{D}}-3.8^{\circ}\left(\mathrm{c} 1.1, \mathrm{CHCl}_{3}\right) ;{ }^{1} \mathrm{H}-\mathrm{NMR}\left(400 \mathrm{MHz}, \mathrm{CDCl}_{3}\right) \delta 8.15-7.45(\mathrm{~m}, 10 \mathrm{H}, 2 \mathrm{Ph})$, $5.65\left(\mathrm{~s}, 1 \mathrm{H}, \mathrm{PhCH} H\right.$ ), 5.43 (near t, $\left.1 \mathrm{H}, J_{2,3}=10.3 \mathrm{~Hz}, J_{1,2}=8.3 \mathrm{~Hz}, \mathrm{H}-2\right), 4.68\left(\mathrm{~d}, 1 \mathrm{H}, J_{1,2}=8.3 \mathrm{~Hz}\right.$, $\mathrm{H}-1), 4.45$ (d, $\left.1 \mathrm{H}, J_{\text {gem }}=12.3 \mathrm{~Hz}, \mathrm{H}-6\right), 4.33$ (d, $\left.1 \mathrm{H}, J_{3,4}=4.2 \mathrm{~Hz}, \mathrm{H}-4\right), 4.18$ (dd, $1 \mathrm{H}, J_{\text {gem }}=12.4 \mathrm{~Hz}$, H-6'), $4.12\left(\mathrm{~m}, 1 \mathrm{H}, \mathrm{OCH}_{2} \mathrm{CH}_{2} \mathrm{SiMe}_{3}\right), 3.97\left(\mathrm{~m}, 1 \mathrm{H}, J_{3, \mathrm{OH}}=11.0 \mathrm{~Hz}, J_{2,3}=10.3 \mathrm{~Hz}, \mathrm{H}-3\right), 3.68-3.60$ (m, $\left.2 \mathrm{H}, \mathrm{H}-5, \mathrm{OCH}_{2} \mathrm{CH}_{2} \mathrm{SiMe}_{3}\right), 2.72(\mathrm{~d}, 1 \mathrm{H}, \mathrm{OH}), 0.98\left(\mathrm{~m}, 2 \mathrm{H}, \mathrm{CH}_{2} \mathrm{CH}_{2} \mathrm{SiMe}_{3}\right), 0.00(\mathrm{~s}, 3 \mathrm{H}$, $\mathrm{CH}_{2} \mathrm{CH}_{2} \mathrm{SiMe}$ ); ${ }^{13} \mathrm{C}-\mathrm{NMR}\left(100 \mathrm{MHz}, \mathrm{CDCl}_{3}\right) \delta 166.2,137.4,132.9,130.0,129.8,129.2,128.2,126.4$, $101.4,100.3,75.6,72.9,71.9,69.0,67.0,66.5,17.9,-1.5 . \mathrm{m} / z$ (MALDI): found $[\mathrm{M}+\mathrm{Na}]^{+} 495.12$, $\mathrm{C}_{25} \mathrm{H}_{32} \mathrm{O}_{7} \mathrm{Si}$ calcd for $[\mathrm{M}+\mathrm{Na}]^{+} 495.18$. 


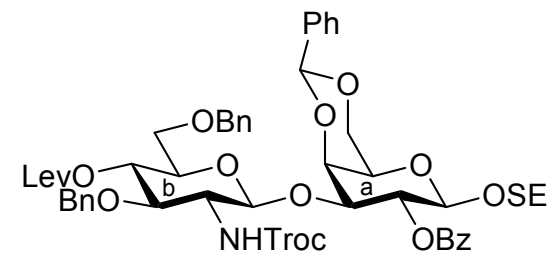

2-(Trimethylsilyl)ethyl 3,6-di-O-benzyl-2-deoxy-4-O-levulinoyl-2-(2,2,2-trichloroethoxycarbamoyl)- $\beta$ D-glucopyranosyl-( $1 \rightarrow 3)$-2-O-benzoyl-4,6-O-benzylidene- $\beta$-D-galactopyranoside (22). To a mixture of 16 (230 mg, $0.317 \mathrm{mmol})$ and $21(100 \mathrm{mg}, 0.212 \mathrm{mmol})$ in $\mathrm{CH}_{2} \mathrm{Cl}_{2}(3.2 \mathrm{~mL})$ was added $4 \AA$ molecular sieves AW-300 (400 mg) at r.t. After stirring for $1 \mathrm{~h}$, the mixture was cooled to $0{ }^{\circ} \mathrm{C}$. NIS (143 $\mathrm{mg}$, $0.636 \mathrm{mmol})$ and $\mathrm{TfOH}(2.8 \mu \mathrm{L}, 31.7 \mu \mathrm{mol})$ were then added to the mixture at $0{ }^{\circ} \mathrm{C}$. After stirring for $2 \mathrm{~h}$ at the same temperature as the reaction was monitored by TLC (1:4 EtOAc-toluene), the reaction was quenched by the addition of triethylamine. The solution was diluted with $\mathrm{CHCl}_{3}$ and filtered through Celite. The filtrate was then washed with satd aq $\mathrm{Na}_{2} \mathrm{~S}_{2} \mathrm{O}_{3}$ and brine. The organic layer was subsequently dried over $\mathrm{Na}_{2} \mathrm{SO}_{4}$, and concentrated. The resulting residue was purified by silica gel column chromatography (1:4 EtOAc-toluene, then $\left.200: 1 \mathrm{CHCl}_{3}-\mathrm{MeOH}\right)$ to give $22(186 \mathrm{mg}, 81 \%)$. $[\alpha]_{\mathrm{D}}+17.4^{\circ}\left(\mathrm{c} 0.8, \mathrm{CHCl}_{3}\right) ;{ }^{1} \mathrm{H}-\mathrm{NMR}\left(600 \mathrm{MHz}, \mathrm{CDCl}_{3}\right) \delta 8.07-7.13(\mathrm{~m}, 20 \mathrm{H}, 4 \mathrm{Ph}), 5.55$ (near t, $\left.1 \mathrm{H}, J_{2,3}=10.3 \mathrm{~Hz}, J_{1,2}=7.6 \mathrm{~Hz}, \mathrm{H}-2 \mathrm{a}\right), 5.49(\mathrm{~s}, 1 \mathrm{H}, \mathrm{PhCH}<), 5.26\left(\mathrm{~d}, 1 \mathrm{H}, J_{\mathrm{NH}, 2}=6.2 \mathrm{~Hz}, \mathrm{NH}\right), 5.08$ (d, $1 \mathrm{H}, J_{1,2}=7.6 \mathrm{~Hz}, \mathrm{H}-1 \mathrm{~b}$ ), 4.92 (near t, $1 \mathrm{H}, J_{3,4}=9.9 \mathrm{~Hz}, J_{4,5}=9.4 \mathrm{~Hz}, \mathrm{H}-4 \mathrm{~b}$ ), 4.53 (d, $1 \mathrm{H}, \mathrm{H}-1 \mathrm{a}$ ), 4.55-4.51 (m, $\left.2 \mathrm{H}, 2 \mathrm{OCH}_{2}\right), 4.46-4.41\left(\mathrm{~m}, 3 \mathrm{H}, 3 \mathrm{OCH}_{2}\right), 4.40\left(\mathrm{~d}, 1 \mathrm{H}, J_{3,4}=3.4 \mathrm{~Hz}, \mathrm{H}-4 \mathrm{a}\right), 4.26(\mathrm{~d}, 1 \mathrm{H}$, $\left.J_{\text {gem }}=12.4 \mathrm{~Hz}, \mathrm{H}-6 \mathrm{a}\right), 4.15$ (t, $\left.1 \mathrm{H}, J_{3,4}=9.9 \mathrm{~Hz}, \mathrm{H}-3 \mathrm{~b}\right), 4.00-3.95$ (m, $2 \mathrm{H}, \mathrm{H}-3 \mathrm{a}, \mathrm{OCH}_{2} \mathrm{CH}_{2} \mathrm{SiMe}_{3}$ ), $3.83\left(\mathrm{~d}, 1 \mathrm{H}, J_{\text {gem }}=12.4 \mathrm{~Hz}, \mathrm{H}-6 \mathrm{a}\right), 3.66$ (br m, $\left.1 \mathrm{H}, \mathrm{H}-5 \mathrm{~b}\right), 3.55$ (m, $3 \mathrm{H}, \mathrm{H}-6 \mathrm{~b}, \mathrm{H}-6$ 'b, $\mathrm{OCH}_{2} \mathrm{CH}_{2} \mathrm{SiMe}_{3}$ ), 3.35 (s, $1 \mathrm{H}, \mathrm{H}-5 \mathrm{a}$ ), 3.28 (br d, $1 \mathrm{H}, \mathrm{OCH}_{2}$ ), 3.17 (br dd, $\left.1 \mathrm{H}, \mathrm{H}-2 \mathrm{~b}\right), 2.61-2.36$ (m, $4 \mathrm{H}, \mathrm{CH}_{2} \mathrm{CH}_{2} \mathrm{C}(=\mathrm{O}) \mathrm{O}$ ), $2.10\left(\mathrm{~s}, 3 \mathrm{H}, \mathrm{C}(=\mathrm{O}) \mathrm{CH}_{3}\right), 0.86-0.76\left(\mathrm{~m}, 2 \mathrm{H}, \mathrm{CH}_{2} \mathrm{CH}_{2} \mathrm{SiMe}_{3}\right),-0.12\left(\mathrm{~m}, 9 \mathrm{H}, \mathrm{CH}_{2} \mathrm{CH}_{2} \mathrm{SiMe}_{3}\right)$; ${ }^{13} \mathrm{C}-\mathrm{NMR}\left(125 \mathrm{MHz}, \mathrm{CDCl}_{3}\right) \delta$ 206.2, 171.6, 164.9, 153.4, 138.1, 138.0, 137.8, 133.0, 130.2, 129.9, $128.9,128.4,128.3,128.3,128.1,127.7,127.6,127.6,127.5,126.5,101.1,100.8,100.2,95.4,78.7$, 77.5, 76.2, 74.2, 73.4, 73.3, 73.2, 71.6, 70.5, 69.9, 68.8, 66.7, 66.6, 58.2, 37.7, 29.7, 27.8, 17.9, -1.6. $\mathrm{m} / \mathrm{z}$ (MALDI): found $[\mathrm{M}+\mathrm{Na}]^{+} 1108.51, \mathrm{C}_{53} \mathrm{H}_{62} \mathrm{Cl}_{3} \mathrm{NO}_{15} \mathrm{Si}$ calcd for $[\mathrm{M}+\mathrm{Na}]^{+} 1108.29$.

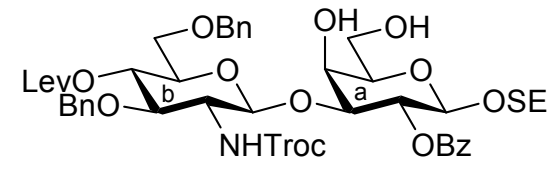

2-(Trimethylsilyl)ethyl 3,6-di-O-benzyl-2-deoxy-4-O-levulinoyl-2-(2,2,2-trichloroethoxycarbamoyl)- $\beta$ D-glucopyranosyl-(1 $\rightarrow 3)$-2-O-benzoyl- $\beta$-D-galactopyranoside (23). Compound 22 (42 mg, $38.3 \mu \mathrm{mol}$ ) was dissolved in $80 \% \mathrm{AcOH}$ aq $(1.5 \mathrm{~mL})$ and the solution was stirred for $4 \mathrm{~h}$ at $50{ }^{\circ} \mathrm{C}$ as the reaction was monitored by TLC $\left(15: 1 \mathrm{CHCl}_{3}-\mathrm{MeOH}\right)$. After the reaction mixture was co-evaporated with toluene, the crude residue was purified by silica gel column chromatography $\left(50: 1 \mathrm{CHCl}_{3}-\mathrm{MeOH}\right)$ to give 23 (38 mg, 99\%). $[\alpha]_{\mathrm{D}}-1.43^{\circ}\left(\mathrm{c} 0.7, \mathrm{CHCl}_{3}\right) ;{ }^{1} \mathrm{H}-\mathrm{NMR}\left(400 \mathrm{MHz}\right.$, acetone- $\left.d_{6}\right) \delta 8.34-7.32(\mathrm{~m}$, $15 \mathrm{H}, 3 \mathrm{Ph}$ ), $7.03\left(\mathrm{~d}, 1 \mathrm{H}, J_{\mathrm{NH}, 2}=9.2 \mathrm{~Hz}, \mathrm{NH}\right), 5.60$ (near t, $1 \mathrm{H}, J_{2,3}=9.7 \mathrm{~Hz}, J_{1,2}=8.0 \mathrm{~Hz}, \mathrm{H}-2 \mathrm{a}$ ), $5.13\left(\mathrm{~d}, 1 \mathrm{H}, J_{1,2}=8.6 \mathrm{~Hz}, \mathrm{H}-1 \mathrm{~b}\right), 5.09$ (near t, $\left.J_{3,4}=9.9 \mathrm{~Hz}, J_{4,5}=8.3 \mathrm{~Hz}, \mathrm{H}-4 \mathrm{~b}\right), 4.77-4.64(\mathrm{~m}, 5 \mathrm{H}$, $\left.\mathrm{H}-1 \mathrm{a}, 4 \mathrm{OCH}_{2}\right), 4.48$ (d, $\left.1 \mathrm{H}, J_{3,4}=3.4 \mathrm{~Hz}, \mathrm{H}-4 \mathrm{a}\right), 4.51$ (d, $\left.1 \mathrm{H}, \mathrm{OCH}_{2}\right), 4.23$ (dd, $\left.1 \mathrm{H}, \mathrm{H}-3 \mathrm{a}\right), 4.13-4.08$ (m, 2 H, H-3b, $\mathrm{OCH}_{2}$ ), 3.96-3.63 (m, 9 H, $2 \mathrm{OCH}_{2}, \mathrm{H}-2 \mathrm{~b}, \mathrm{H}-5 \mathrm{~b}, \mathrm{H}-6 \mathrm{~b}, \mathrm{H}-6$ 'b , H-5a, H-6a, H-6'a), 2.98 (s, $2 \mathrm{H}, 2 \mathrm{OH}), 2.80-2.49\left(\mathrm{~m}, 4 \mathrm{H}, \mathrm{CH}_{2} \mathrm{CH}_{2} \mathrm{C}(=\mathrm{O}) \mathrm{O}\right), 2.20\left(\mathrm{~s}, 3 \mathrm{H}, \mathrm{C}(=\mathrm{O}) \mathrm{CH}_{3}\right), 1.01-0.83(\mathrm{~m}, 2 \mathrm{H}$, $\mathrm{CH}_{2} \mathrm{CH}_{2} \mathrm{SiMe}_{3}$ ), $0.00\left(\mathrm{~s}, 9 \mathrm{H}, \mathrm{CH}_{2} \mathrm{CH}_{2} \mathrm{SiMe}\right.$ ) $;{ }^{13} \mathrm{C}-\mathrm{NMR}\left(100 \mathrm{MHz}\right.$, acetone- $\left.d_{6}\right) \delta 206.5,172.5,165.6$, 
$154.8,139.5,133.7,131.7,130.7,129.2,129.0,128.8,128.4,128.3,128.2,128.0,102.7,101.7,96.8$, 82.4, 80.2, 75.9, 74.6, 74.1, 74.0, 73.9, 72.1, 72.0, 70.4, 69.5, 67.0, 62.6, 58.3, 38.1, 29.6, 28.6, 18.6, -1.4. $m / z$ (MALDI): found $[\mathrm{M}+\mathrm{Na}]^{+} 1020.26, \mathrm{C}_{46} \mathrm{H}_{58} \mathrm{Cl}_{3} \mathrm{NO}_{15} \mathrm{Si}$ calcd for $[\mathrm{M}+\mathrm{Na}]^{+} 1020.25$.

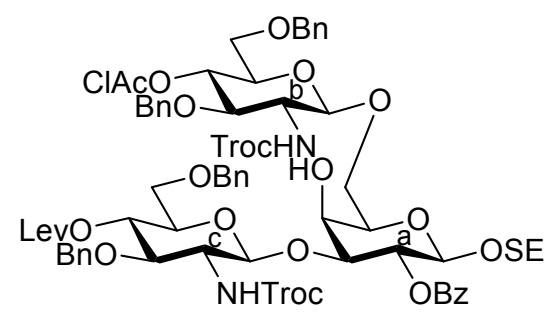

2-(Trimethylsilyl)ethyl 3,6-di-O-benzyl-2-deoxy-4-O-levulinoyl-2-(2,2,2-trichloroethoxycarbamoyl)- $\beta$ D-glucopyranosyl-(1 $\rightarrow 3)$-[3,6-di-O-benzyl-4-O-chloroacetyl-2-deoxy-2-(2,2,2-trichloroethoxycarbamoyl)-

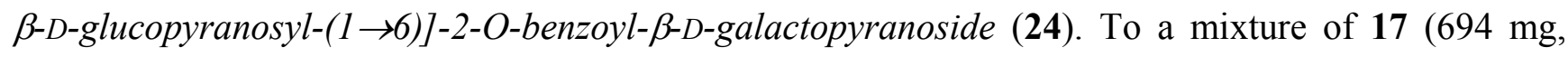
$0.987 \mathrm{mmol})$ and $23(658 \mathrm{mg}, 0.658 \mathrm{mmol})$ in $\mathrm{CH}_{2} \mathrm{Cl}_{2}(6.6 \mathrm{~mL})$ was added $4 \AA$ molecular sieves $(1.40 \mathrm{~g})$ at r.t. After stirring for $1 \mathrm{~h}$, the mixture was cooled to $0{ }^{\circ} \mathrm{C}$. NIS (296 $\left.\mathrm{mg}, 1.32 \mathrm{mmol}\right)$ and $\mathrm{TfOH}(8.7 \mu \mathrm{L}, 98.7 \mu \mathrm{mol})$ were then added to the mixture at $0{ }^{\circ} \mathrm{C}$. After stirring for $2 \mathrm{~h}$ at r.t. as the reaction was monitored by TLC $\left(30: 1 \mathrm{CHCl}_{3}-\mathrm{MeOH}\right)$, the reaction was quenched by the addition of triethylamine. The solution was diluted with $\mathrm{CHCl}_{3}$ and filtered through Celite. The filtrate was then washed with satd aq $\mathrm{Na}_{2} \mathrm{~S}_{2} \mathrm{O}_{3}$ and brine. The organic layer was subsequently dried over $\mathrm{Na}_{2} \mathrm{SO}_{4}$, and concentrated. The residue was purified by silica gel column chromatography $\left(100: 1 \mathrm{CHCl}_{3}-\mathrm{MeOH}\right)$ to give 24 (511 mg, 49\%). $[\alpha]_{\mathrm{D}}-19.4^{\circ}\left(\mathrm{c} 1.8, \mathrm{CHCl}_{3}\right)$; ${ }^{1} \mathrm{H}-\mathrm{NMR}\left(400 \mathrm{MHz}, \mathrm{DMSO}-d_{6}\right) \delta 8.20-7.32(\mathrm{~m}$, $27 \mathrm{H}, 5 \mathrm{Ph}, \mathrm{NHb}, \mathrm{NHc}), 5.38$ (t, $\left.1 \mathrm{H}, J_{2,3}=8.3 \mathrm{~Hz}, J_{1,2}=8.7 \mathrm{~Hz}, \mathrm{H}-2 \mathrm{a}\right), 5.14$ (t, $1 \mathrm{H}, J_{4,5}=9.6 \mathrm{~Hz}$, $\mathrm{H}-4 \mathrm{c}), 5.06\left(\mathrm{~d}, 1 \mathrm{H}, J_{\text {gem }}=12.4 \mathrm{~Hz}, \mathrm{OCH}_{2}\right), 5.01\left(\mathrm{t}, 1 \mathrm{H}, J_{3,4}=9.6 \mathrm{~Hz}, J_{4,5}=9.1 \mathrm{~Hz}, \mathrm{H}-4 \mathrm{~b}\right), 4.90(\mathrm{~d}$, $\left.1 \mathrm{H}, J_{1,2}=8.2 \mathrm{~Hz}, \mathrm{H}-1 \mathrm{c}\right), 4.75$ (d, $\left.1 \mathrm{H}, J_{1,2}=8.3 \mathrm{~Hz}, \mathrm{H}-1 \mathrm{~b}\right), 4.66$ (d, $\left.1 \mathrm{H}, \mathrm{H}-1 \mathrm{a}\right), 4.91-4.61$ (m, $10 \mathrm{H}$, $\mathrm{OH}, 9 \mathrm{OCH}_{2}$ ), 4.49 (2 d, 2 H, OCH ), 4.24 (br s, $\left.1 \mathrm{H}, \mathrm{H}-4 \mathrm{a}\right), 4.04-3.52$ (m, 18 H, H-3a, H-5a, H-6a, H-6'a, H-2b, H-3b, H-5b, H-6b, H-6'b, H-2c, H-3c, H-5c, H-6c, H-6'c, OCH $\mathrm{CH}_{2} \mathrm{SiMe}_{3}, \mathrm{CH}_{2} \mathrm{Cl}$ ), 2.83-2.80 (m, $\left.2 \mathrm{H}, \mathrm{CH}_{2} \mathrm{CH}_{2} \mathrm{C}(=\mathrm{O}) \mathrm{O}\right), 2.56\left(\mathrm{~m}, 2 \mathrm{H}, \mathrm{CH}_{2} \mathrm{CH}_{2} \mathrm{C}(=\mathrm{O}) \mathrm{O}\right), 2.26\left(\mathrm{~s}, 3 \mathrm{H}, \mathrm{C}(=\mathrm{O}) \mathrm{CH}_{3}\right)$, 0.93-0.78 (m, $\left.2 \mathrm{H}, \mathrm{CH}_{2} \mathrm{CH}_{2} \mathrm{SiMe}_{3}\right), 0.00\left(\mathrm{~s}, 9 \mathrm{H}, \mathrm{CH}_{2} \mathrm{CH}_{2} \mathrm{SiMe} 3\right) ;{ }^{13} \mathrm{C}-\mathrm{NMR}\left(100 \mathrm{MHz}, \mathrm{DMSO}-d_{6}\right) \delta$ 206.8, 171.5, 166.5, 164.7, 154.4, 153.8, 138.4, 138.3, 138.2, 133.2, 130.3, 129.6, 128.6, 128.4, 128.3, $128.2,127.7,127.7,127.6,101.9,101.1,100.2,96.4,96.0,80.7,79.4,78.9,73.7,73.6,73.3,72.8$, 72.7, 72.6, 72.5, 72.2, 72.0, 70.9, 70.7, 69.6, 69.0, 68.7, 68.6, 66.1, 57.0, 41.1, 37.4, 29.7, 27.8, 17.6, -1.3. $m / z$ (MALDI): found [M+Na] ${ }^{+} 1611.27, \mathrm{C}_{71} \mathrm{H}_{83} \mathrm{Cl}_{7} \mathrm{~N}_{2} \mathrm{O}_{22} \mathrm{Si}$ calcd for $[\mathrm{M}+\mathrm{Na}]^{+} 1611.29$.

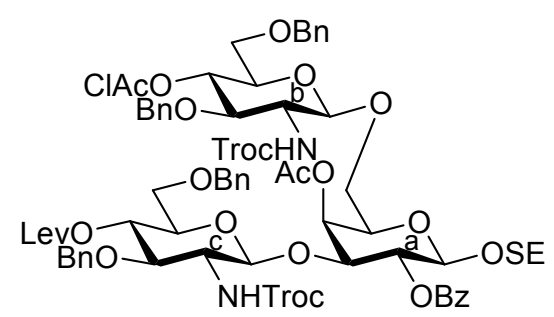

2-(Trimethylsilyl)ethyl 3,6-di-O-benzyl-2-deoxy-4-O-levulinoyl-2-(2,2,2-trichloroethoxycarbamoyl)- $\beta$ D-glucopyranosyl-(1 $\rightarrow 3)$-[3,6-di-O-benzyl-4-O-chloroacetyl-2-deoxy-2-(2,2,2-trichloroethoxycarbamoyl)-

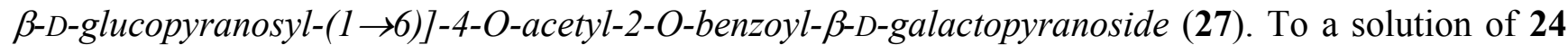
(759 mg, $0.476 \mathrm{mmol})$ in THF $(4.8 \mathrm{~mL})$ were added acetic anhydride $(94 \mu \mathrm{L}, 0.956 \mathrm{mmol})$ and DMAP $(5.8 \mathrm{mg}, 47.6 \mu \mathrm{mol})$ at $0{ }^{\circ} \mathrm{C}$. The reaction mixture was stirred for $1.5 \mathrm{~h}$ at r.t. as the reaction was 
monitored by TLC (15:1 $\left.\mathrm{CHCl}_{3}-\mathrm{MeOH}\right)$. After THF was evaporated, the obtained crude residue was purified by silica gel column chromatography $\left(100: 1 \mathrm{CHCl}_{3}-\mathrm{MeOH}\right)$ to give $27(745 \mathrm{mg}, 96 \%)$. $[\alpha]_{\mathrm{D}}$ $-5.5^{\circ}$ (c 1.8, $\mathrm{CHCl}_{3}$ ); ${ }^{1} \mathrm{H}-\mathrm{NMR}\left(400 \mathrm{MHz}, \mathrm{DMSO}-d_{6}\right) \delta 8.20-7.32$ (m, $\left.27 \mathrm{H}, 5 \mathrm{Ph}, \mathrm{NHb}, \mathrm{NHc}\right), 5.58$ (br s, $1 \mathrm{H}, \mathrm{H}-4 \mathrm{a}$ ), 5.34 (near t, $1 \mathrm{H}, J_{2,3}=9.7 \mathrm{~Hz}, J_{1,2}=8.2 \mathrm{~Hz}, \mathrm{H}-2 \mathrm{a}$ ), 5.16-5.07 (m, $3 \mathrm{H}, \mathrm{H}-4 \mathrm{c}, \mathrm{H}-4 \mathrm{~b}$, $\mathrm{OCH}_{2}$ ), 4.86-4.61 (m, $\left.10 \mathrm{H}, \mathrm{H}-1 \mathrm{~b}, \mathrm{H}-1 \mathrm{c}, \mathrm{H}-1 \mathrm{a}, 7 \mathrm{OCH}_{2}\right), 4.58-4.39$ (m, $3 \mathrm{H}, 3 \mathrm{OCH}_{2}$ ), 4.31 (br dd, 1 H, H-3a), 4.18 (br dd, 1 H, H-6a), 4.06-3.48 (m, 17 H, H-5a, H-6'a, H-2b, H-3b, H-5b, H-6b, H-6'b, H-2c, H-3c, H-5c, H-6c, H-6'c, $\left.\mathrm{OCH}_{2}, \mathrm{OCH}_{2} \mathrm{CH}_{2} \mathrm{SiMe}_{3}, \mathrm{CH}_{2} \mathrm{Cl}\right), 2.80$ (m, $\left.2 \mathrm{H}, \mathrm{CH}_{2} \mathrm{CH}_{2} \mathrm{C}(=\mathrm{O}) \mathrm{O}\right), 2.54$ $\left(\mathrm{m}, 2 \mathrm{H}, \mathrm{CH}_{2} \mathrm{CH}_{2} \mathrm{C}(=\mathrm{O}) \mathrm{O}\right), 2.25-2.22\left(2 \mathrm{~s}, 6 \mathrm{H}, 2 \mathrm{C}(=\mathrm{O}) \mathrm{CH}_{3}\right), 0.96-0.79\left(\mathrm{~m}, 2 \mathrm{H}, \mathrm{CH}_{2} \mathrm{CH}_{2} \mathrm{SiMe}_{3}\right)$, $0.00\left(\mathrm{~s}, 9 \mathrm{H}, \mathrm{CH}_{2} \mathrm{CH}_{2} \mathrm{SiMe}\right.$ ) $){ }^{13} \mathrm{C}-\mathrm{NMR}\left(100 \mathrm{MHz}, \mathrm{DMSO}-d_{6}\right) \delta 206.7,179.5,171.3,170.0,166.5$, $164.5,154.4,153.8,138.5,138.3,138.3,138.2,133.3,130.1,129.6,128.7,128.4,128.3,128.3,128.1$, 127.7, 127.6, 127.6, 127.5, 127.2, 101.3, 100.9, 100.0, 96.3, 96.0, 79.3, 79.0, 77.6, 73.6, 73.1, 72.7, 72.4, 72.3, 72.0, 71.1, 70.3, 70.2, 69.0, 68.8, 68.4, 66.4, 57.0, 41.1, 37.4, 29.7, 27.8, 20.8, 17.6, -1.3. $\mathrm{m} / \mathrm{z}$ (MALDI): found [M+Na] ${ }^{+} 1653.57, \mathrm{C}_{73} \mathrm{H}_{85} \mathrm{Cl}_{7} \mathrm{~N}_{2} \mathrm{O}_{23} \mathrm{Si}$ calcd for $[\mathrm{M}+\mathrm{Na}]^{+} 1653.30$.

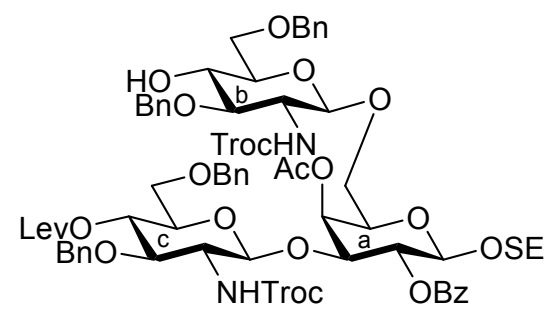

2-(Trimethylsilyl)ethyl 3,6-di-O-benzyl-2-deoxy-4-O-levulinoyl-2-(2,2,2-trichloroethoxycarbamoyl)$\beta$-D-glucopyranosyl-(1 $\rightarrow 3)$-[3, 6-di-O-benzyl-2-deoxy-2-(2,2,2-trichloroethoxycarbamoyl)- $\beta$ - $D$ glucopyranosyl-(1-6)]-4-O-acetyl-2-O-benzoyl- $\beta$-D-galactopyranoside (28). To a solution of 27 (712 $\mathrm{mg}$, $0.435 \mathrm{mmol})$ in EtOH $(4.4 \mathrm{~mL})$ was added DABCO $(732 \mathrm{mg}, 6.52 \mathrm{mmol})$ at $0{ }^{\circ} \mathrm{C}$. After stirring for $1 \mathrm{~h}$ at $50{ }^{\circ} \mathrm{C}$ as the reaction was monitored by TLC $\left(30: 1 \mathrm{CHCl}_{3}-\mathrm{MeOH}\right)$, EtOH was evaporated. The obtained crude residue was purified by silica gel column chromatography (100:1 $\left.\mathrm{CHCl}_{3}-\mathrm{MeOH}\right)$ to give $28(665 \mathrm{mg}, 98 \%)$. $[\alpha]_{\mathrm{D}}-1.8^{\circ}\left(\mathrm{c} 1.5, \mathrm{CHCl}_{3}\right) ;{ }^{1} \mathrm{H}-\mathrm{NMR}\left(500 \mathrm{MHz}, \mathrm{CDCl}_{3}\right) \delta 8.19-7.27(\mathrm{~m}, 25 \mathrm{H}$, $5 \mathrm{Ph}$ ), $5.63\left(\mathrm{~d}, 1 \mathrm{H}, J_{3,4}=3.4 \mathrm{~Hz}, \mathrm{H}-4 \mathrm{a}\right), 5.52$ (near t, $\left.1 \mathrm{H}, J_{2,3}=10.3 \mathrm{~Hz}, J_{1,2}=8.0 \mathrm{~Hz}, \mathrm{H}-2 \mathrm{a}\right), 5.32$ (br $\mathrm{d}, 1 \mathrm{H}, \mathrm{NHb}), 5.18\left(\mathrm{~d}, 1 \mathrm{H}, J_{\mathrm{NH}, 2}=6.9 \mathrm{~Hz}, \mathrm{NHc}\right), 5.07\left(\mathrm{~d}, 1 \mathrm{H}, J_{1,2}=9.2 \mathrm{~Hz}, \mathrm{H}-1 \mathrm{c}\right), 5.05$ (near t, $1 \mathrm{H}$, $\left.J_{3,4}=9.2 \mathrm{~Hz}, \mathrm{H}-4 \mathrm{c}\right), 4.85\left(\mathrm{~d}, 1 \mathrm{H}, J_{1,2}=8.6 \mathrm{~Hz}, \mathrm{H}-1 \mathrm{~b}\right), 4.91-4.80\left(\mathrm{~m}, 3 \mathrm{H}, 3 \mathrm{OCH}_{2}\right), 4.71(\mathrm{~d}, 1 \mathrm{H}$, $\left.\mathrm{OCH}_{2}\right), 4.63$ (d, $\left.1 \mathrm{H}, \mathrm{H}-1 \mathrm{a}\right), 4.69-4.59\left(\mathrm{~m}, 6 \mathrm{H}, 6 \mathrm{OCH}_{2}\right), 4.50\left(\mathrm{~d}, 1 \mathrm{H}, \mathrm{OCH}_{2}\right), 4.16$ (br t, $1 \mathrm{H}$, $\left.J_{2,3}=10.3 \mathrm{~Hz}, \mathrm{H}-3 \mathrm{c}\right), 4.09$ (m, $1 \mathrm{H}, \mathrm{OCH}_{2} \mathrm{CH}_{2} \mathrm{SiMe}_{3}$ ), 4.07 (dd, $\left.1 \mathrm{H}, \mathrm{H}-3 \mathrm{a}\right), 4.01$ (dd, $1 \mathrm{H}, J_{5,6}=4.1 \mathrm{~Hz}$, $J_{\text {gem }}=12.8$ Hz, H-6a), 3.89-3.56 (m, 11 H, H-5a, H-6'a, H-3b, H-5b, H-6b, H-6'b, H-5c, H-6c, H-6'c, $\mathrm{OCH}_{2}, \mathrm{OCH}_{2} \mathrm{CH}_{2} \mathrm{SiMe}_{3}$ ), 3.44 (br dd, $\left.1 \mathrm{H}, \mathrm{H}-2 \mathrm{~b}\right), 3.23$ (br dd, $1 \mathrm{H}, \mathrm{H}-2 \mathrm{c}$ ), 2.96 (s, $\left.1 \mathrm{H}, \mathrm{OH}\right), 2.72-2.37$ $\left(\mathrm{m}, 4 \mathrm{H}, \mathrm{CH}_{2} \mathrm{CH}_{2} \mathrm{C}(=\mathrm{O}) \mathrm{O}\right), 2.22\left(\mathrm{~m}, 6 \mathrm{H}, 2 \mathrm{C}(=\mathrm{O}) \mathrm{CH}_{3}\right), 1.01-0.86\left(\mathrm{~m}, 2 \mathrm{H}, \mathrm{CH}_{2} \mathrm{CH}_{2} \mathrm{SiMe}_{3}\right), 0.00$ (s, $9 \mathrm{H}, \mathrm{CH}_{2} \mathrm{CH}_{2} \mathrm{SiMe}$ ); ${ }^{13} \mathrm{C}-\mathrm{NMR}\left(100 \mathrm{MHz}, \mathrm{CDCl}_{3}\right) \delta$ 206.1, 171.4, 169.9, 164.8, 154.0, 153.3, 138.2, $138.1,137.7,137.5,133.2$, 129.9, 128.5, 128.4, 128.3, 128.0, 127.9, 127.8, 127.7, 127.6, 127.6, 100.7, 100.2, 95.6, 95.4, 80.5, 77.5, 74.3, 74.3, 73.9, 73.7, 73.4, 73.4, 73.2, 73.1, 71.5, 71.4, 70.5, 70.1, 69.6, 67.4, 57.9, 57.3, 37.6, 29.6, 27.8, 20.8, 17.9, -1.5. $\mathrm{m} / \mathrm{z}$ (MALDI): found $[\mathrm{M}+\mathrm{Na}]^{+} 1577.28$, $\mathrm{C}_{71} \mathrm{H}_{84} \mathrm{Cl}_{6} \mathrm{~N}_{2} \mathrm{O}_{22} \mathrm{Si}$ calcd for $[\mathrm{M}+\mathrm{Na}]^{+} 1577.33$. 


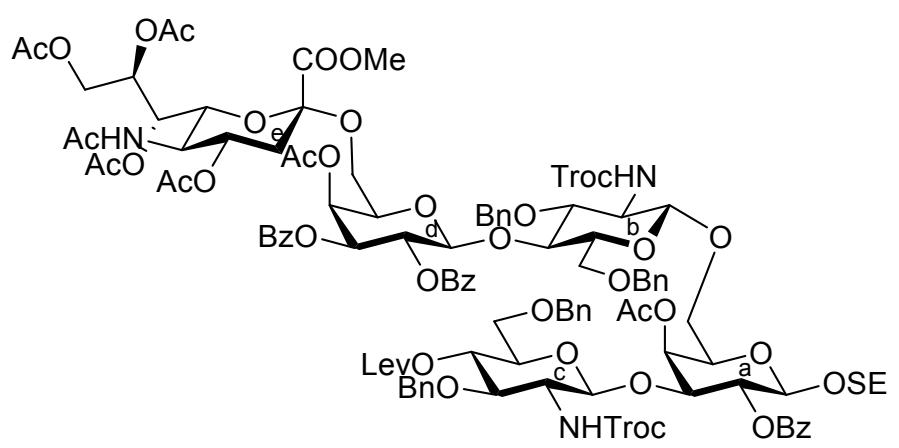

2-(Trimethylsilyl)ethyl (methyl 5-acetamido-4,7,8,9-tetra-O-acetyl-3,5-dideoxy-D-glycero- $\alpha$-D-galacto-2nonulopyranosylonate)-(2 $\rightarrow 6)-4-O$-acetyl-2,3-di-O-benzoyl- $\beta$-D-galactopyranosyl-( $1 \rightarrow 4)$-3, 6-di-Obenzyl-2-deoxy-2-(2,2,2-trichloroethoxycarbamoyl)- $\beta$-D-glucopyranosyl-(1 $\rightarrow 6)$-[3,6-di-O-benzyl-2deoxy-4-O-levulinoyl-2-(2,2,2-trichloroethoxycarbamoyl)- $\beta$-D-glucopyranosyl-( $1 \rightarrow 3)]$-4-O-acetyl-2O-benzoyl- $\beta$-D-galactopyranoside (29). To a mixture of $12(80 \mathrm{mg}, 76.5 \mu \mathrm{mol})$ and 28 (70 $\mathrm{mg}$, $45.0 \mu \mathrm{mol})$ in $\mathrm{CH}_{2} \mathrm{Cl}_{2}(0.9 \mathrm{~mL})$ was added $3 \AA$ molecular sieves $(250 \mathrm{mg})$ at r.t. After stirring for $1 \mathrm{~h}$, the mixture was cooled to $0{ }^{\circ} \mathrm{C}$. TMSOTf $(1.4 \mu \mathrm{L}, 7.65 \mu \mathrm{mol})$ was then added to the mixture at $0{ }^{\circ} \mathrm{C}$. After stirring for $25 \mathrm{~h}$ at r.t., TMSOTf $(1.4 \mu \mathrm{L}, 7.65 \mu \mathrm{mol})$ was added to the mixture. After the stirring was continued for $3 \mathrm{~h}$ at r.t. as the reaction was monitored by TLC $\left(30: 1 \mathrm{CHCl}_{3}-\mathrm{MeOH}\right)$, the reaction was quenched by the addition of triethylamine. The mixture was diluted with $\mathrm{CHCl}_{3}$ and filtered through Celite. The filtrate was then washed with satd aq $\mathrm{NaHCO}_{3}$ and brine. The organic layer was subsequently dried over $\mathrm{Na}_{2} \mathrm{SO}_{4}$, and concentrated. The resulting residue was purified by silica gel column chromatography $\left(90: 1 \mathrm{CHCl}_{3}-\mathrm{MeOH}\right)$ followed by gel filtration column chromatography (LH-20) using $\mathrm{MeOH}$ as eluent, giving 29 (81 mg, 74\%). $[\alpha]_{\mathrm{D}}-2.9^{\circ}$ (c 1.4, $\mathrm{CHCl}_{3}$ ); ${ }^{1} \mathrm{H}-\mathrm{NMR}(600 \mathrm{MHz}$, $\left.\mathrm{CDCl}_{3}\right) \delta 8.05-7.14(\mathrm{~m}, 35 \mathrm{H}, 7 \mathrm{Ph}), 5.61\left(\mathrm{~d}, 1 \mathrm{H}, J_{3,4}=3.3 \mathrm{~Hz}, \mathrm{H}-4 \mathrm{~d}\right), 5.59$ (near t, $1 \mathrm{H}, J_{1,2}=8.3 \mathrm{~Hz}$, H-2d), 5.45 (d, $\left.1 \mathrm{H}, J_{3,4}=3.4 \mathrm{~Hz}, \mathrm{H}-4 \mathrm{a}\right), 5.40-5.36$ (m, $\left.1 \mathrm{H}, \mathrm{H}-8 \mathrm{e}\right), 5.36$ (t, $\left.1 \mathrm{H}, J_{1,2}=8.2 \mathrm{~Hz}, \mathrm{H}-2 \mathrm{a}\right)$, 5.34 (m, $1 \mathrm{H}, \mathrm{H}-7 \mathrm{e}), 5.30$ (dd, $\left.1 \mathrm{H}, J_{2,3}=10.2 \mathrm{~Hz}, \mathrm{H}-3 \mathrm{~d}\right), 5.23$ (2 br d, $\left.2 \mathrm{H}, \mathrm{NHe}, \mathrm{NHb}\right), 5.02$ (d, $1 \mathrm{H}$, $\left.J_{2, \mathrm{NH}}=6.9 \mathrm{~Hz}, \mathrm{NHc}\right), 5.12\left(\mathrm{~d}, 1 \mathrm{H}, J_{\mathrm{gem}}=11.0 \mathrm{~Hz}, \mathrm{OCH}_{2}\right), 4.94(\mathrm{~d}, 1 \mathrm{H}, \mathrm{H}-1 \mathrm{~d}), 4.94-4.86(\mathrm{~m}, 2 \mathrm{H}, \mathrm{H}-1 \mathrm{c}$, $\mathrm{H}-4 \mathrm{c}), 4.84\left(\mathrm{~m}, 1 \mathrm{H}, J_{3 \mathrm{eq}, 4}=4.2 \mathrm{~Hz}, \mathrm{H}-4 \mathrm{e}\right), 4.73-4.68\left(\mathrm{~m}, 3 \mathrm{H}, 3 \mathrm{OCH}_{2}\right), 4.58-4.30(\mathrm{~m}, 10 \mathrm{H}, \mathrm{H}-1 \mathrm{a}$, H-1b, H-9e, $7 \mathrm{OCH}_{2}$ ), 4.14-3.71 (m, 15 H, H-3a, H-6'a, H-3b, H-4b, H-3c, H-4c, H-5d, H-6d, H-6'd, H-5e, H-6e, $\mathrm{OCH}_{2}, \mathrm{OCH}_{3}$ ), 3.58-3.37 (m, 10 H, H-6a, H-2b, H-5b, H-6b, H-6'b, H-5c, H-6c, H-6'c, $\left.2 \mathrm{OCH}_{2}\right), 3.28-3.26(\mathrm{~m}, 1 \mathrm{H}, \mathrm{H}-5 \mathrm{a}), 3.08$ (br dd, $\left.1 \mathrm{H}, \mathrm{H}-2 \mathrm{c}\right), 2.60-2.51$ (m, $3 \mathrm{H}, \mathrm{H}-3 \mathrm{e} e q$, $\left.\mathrm{CH}_{2} \mathrm{CH}_{2} \mathrm{C}(=\mathrm{O}) \mathrm{O}\right), 2.38-2.24\left(\mathrm{~m}, 2 \mathrm{H}, \mathrm{CH}_{2} \mathrm{CH}_{2} \mathrm{C}(=\mathrm{O}) \mathrm{O}\right), 2.16-1.91\left(\mathrm{~m}, 25 \mathrm{H}, 8 \mathrm{C}(=\mathrm{O}) \mathrm{CH}_{3}, \mathrm{H}-3 \mathrm{e} a x\right)$, 0.85-0.69 (m, $\left.2 \mathrm{H}, \mathrm{CH}_{2} \mathrm{CH}_{2} \mathrm{SiMe}_{3}\right),-0.14\left(\mathrm{~s}, 9 \mathrm{H}, \mathrm{CH}_{2} \mathrm{CH}_{2} \mathrm{SiMe}_{3}\right) ;{ }^{13} \mathrm{C}-\mathrm{NMR}\left(100 \mathrm{MHz}, \mathrm{CDCl}_{3}\right) \delta$ 206.0, 171.4, 170.9, 170.7, 170.3, 170.1, 169.8, 169.6, 167.8, 165.3, 165.2, 164.8, 153.9, 153.3, 138.6, $138.1,138.0,137.7,133.3,133.1,129.8,129.6,129.5,129.1,128.5,128.4,128.4,128.3,128.3,128.2$, $127.9,127.8,127.7,127.6,127.6,127.4,100.6,100.1,99.8,99.0,95.4,77.4,76.8,76.1,74.4,74.3$, $73.9,73.7,73.3,73.3,73.1,72.8,71.7,71.5,71.4,70.2,70.1,69.6,68.7,68.4,68.1,67.3,62.4,57.9$, 57.0, 52.9, 49.4, 37.7, 37.6, 29.6, 27.8, 23.1, 21.0, 20.8, 20.5, 17.8, -1.5. $\mathrm{m} / \mathrm{z}$ (MALDI): found $[\mathrm{M}+\mathrm{Na}]^{+} 2463.09, \mathrm{C}_{113} \mathrm{H}_{131} \mathrm{Cl}_{6} \mathrm{~N}_{3} \mathrm{O}_{42} \mathrm{Si}$ calcd for $[\mathrm{M}+\mathrm{Na}]^{+} 2462.60$. 


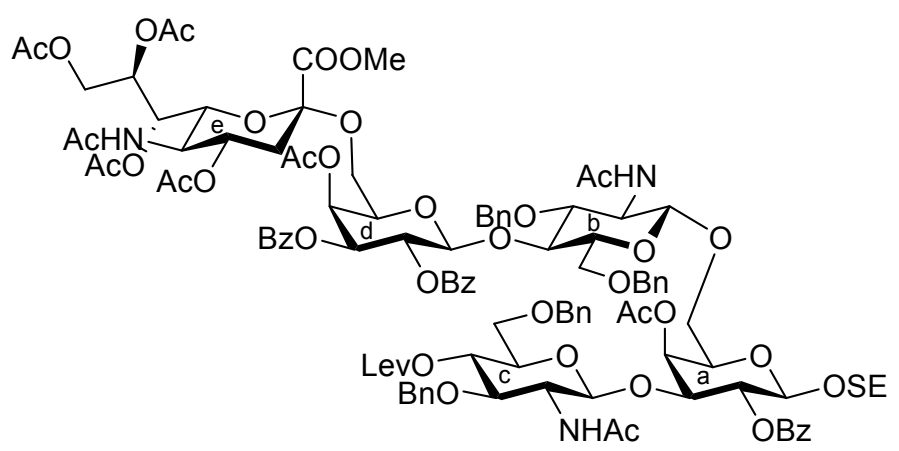

2-(Trimethylsilyl)ethyl (methyl 5-acetamido-4,7,8,9-tetra-O-acetyl-3,5-dideoxy-D-glycero- $\alpha$-D-galacto-2nonulopyranosylonate)-(2 $\rightarrow 6)-4-O$-acetyl-2,3-di-O-benzoyl- $\beta$-D-galactopyranosyl- $(1 \rightarrow 4)$-2-acetamido3,6-di-O-benzyl-2-deoxy- $\beta$-D-glucopyranosyl- $(1 \rightarrow 6)$-[2-acetamido-3, 6-di-O-benzyl-2-deoxy-4O-levulinoyl- $\beta$-D-glucopyranosyl-(1 $\rightarrow 3)]$-4-O-acetyl-2-O-benzoyl- $\beta$-D-galactopyranoside (31). To a solution of $29(220 \mathrm{mg}, 89.9 \mu \mathrm{mol})$ in $\mathrm{CH}_{2} \mathrm{Cl}_{2} / \mathrm{AcOH}(2: 3,3.5 \mathrm{~mL})$ was added $\mathrm{Zn} / \mathrm{Cu}$ couple $(2.20 \mathrm{~g})$ at r.t. The reaction mixture was heated to $50{ }^{\circ} \mathrm{C}$ and was stirred for $2.5 \mathrm{~h}$ at the same temperature as the reaction was monitored by TLC $\left(15: 1 \mathrm{CHCl}_{3}-\mathrm{MeOH}\right)$. The precipitate was filtered through Celite and the filtrate was co-evaporated with toluene. The obtained residue was exposed to high vacuum for $6 \mathrm{~h}$. The crude residue was dissolved in pyridine $(3.6 \mathrm{~mL})$ and acetic anhydride $(68 \mu \mathrm{L}, 0.719 \mathrm{mmol})$, DMAP $(2.5 \mathrm{mg}, 47.6 \mu \mathrm{mol})$ were then added to the mixture at $0{ }^{\circ} \mathrm{C}$. After stirring for $12 \mathrm{~h}$ at r.t as the reaction was monitored by $\mathrm{TLC}\left(15: 1 \mathrm{CHCl}_{3}-\mathrm{MeOH}\right)$, the reaction mixture was evaporated. The obtained residue was diluted with $\mathrm{CHCl}_{3}$ and washed with $2 \mathrm{M} \mathrm{HCl}$, satd aq $\mathrm{NaHCO}_{3}$, and brine, dried over $\mathrm{Na}_{2} \mathrm{SO}_{4}$, and concentrated. The resulting residue was purified by silica gel column chromatography $\left(40: 1 \mathrm{CHCl}_{3}-\mathrm{MeOH}\right)$ to give $31(120 \mathrm{mg}, 61 \%)$. $[\alpha]_{\mathrm{D}}-1.9^{\circ}\left(\mathrm{c} 0.5, \mathrm{CHCl}_{3}\right) ;{ }^{1} \mathrm{H}-\mathrm{NMR}$ $\left(600 \mathrm{MHz}, \mathrm{CDCl}_{3}\right) \delta 8.02-7.13(\mathrm{~m}, 35 \mathrm{H}, 7 \mathrm{Ph}), 5.93\left(\mathrm{~d}, 1 \mathrm{H}, J_{2, \mathrm{NH}}=8.3 \mathrm{~Hz}, \mathrm{NHb}\right), 5.63(\mathrm{~d}, 1 \mathrm{H}$, $J_{3,4}=3.4 \mathrm{~Hz}, \mathrm{H}-4 \mathrm{~d}$ ), 5.56 (near t, $1 \mathrm{H}, J_{1,2}=7.5 \mathrm{~Hz}, J_{2,3}=8.2 \mathrm{~Hz}, \mathrm{H}-2 \mathrm{~d}$ ), $5.41-5.31$ (m, $5 \mathrm{H}, \mathrm{H}-2 \mathrm{a}, \mathrm{H}-4 \mathrm{a}$, H-3d, H-7e, H-8e), 5.26 (d, $\left.1 \mathrm{H}, J_{2, \mathrm{NH}}=7.6 \mathrm{~Hz}, \mathrm{NHc}\right), 5.13\left(\mathrm{~d}, 1 \mathrm{H}, J_{5, \mathrm{NH}}=7.5 \mathrm{~Hz}, \mathrm{NHe}\right), 4.95(\mathrm{~d}, 1 \mathrm{H}$, $\left.J_{1,2}=8.2 \mathrm{~Hz}, \mathrm{H}-1 \mathrm{c}\right), 4.90-4.83\left(\mathrm{~m}, 4 \mathrm{H}, \mathrm{H}-4 \mathrm{c}, \mathrm{H}-1 \mathrm{~d}, \mathrm{H}-4 \mathrm{e}, \mathrm{OCH}_{2}\right), 4.70\left(\mathrm{~d}, 1 \mathrm{H}, J_{\text {gem }}=11.7 \mathrm{~Hz}\right.$, $\left.\mathrm{OCH}_{2}\right), 4.58\left(\mathrm{~d}, 1 \mathrm{H}, J_{1,2}=6.9 \mathrm{~Hz}, \mathrm{H}-1 \mathrm{c}\right), 4.44\left(\mathrm{~d}, 1 \mathrm{H}, J_{1,2}=8.3 \mathrm{~Hz}, \mathrm{H}-1 \mathrm{a}\right), 4.54-4.31$ (m, $7 \mathrm{H}, \mathrm{H}-9 \mathrm{e}$, $3 \mathrm{OCH}_{2}$ ), 4.13 (t, $1 \mathrm{H}, J_{2,3}=9.6 \mathrm{~Hz}, \mathrm{H}-3 \mathrm{c}$ ), 4.10-4.02 (m, 4 H, H-6a, H-4b, H-5e, H-9e), 3.97-3.75 (m, 9 H, H-3a, H-6'a, H-3b, H-6d, H-6e, $\mathrm{OCH}_{2} \mathrm{CH}_{2} \mathrm{SiMe}_{3}, \mathrm{OCH}_{3}$ ), 3.67-3.35 (m, 11 H, H-5a, H-2b, H-5b, H-6b, H-6'b, H-5c, H-6c, H-6'c, H-5d, H-6'd, OCH $\mathrm{CH}_{2} \mathrm{SiMe}_{3}$ ), 3.07 (dd, $1 \mathrm{H}, \mathrm{H}-2 \mathrm{c}$ ), 2.54-2.51 (m, $\left.3 \mathrm{H}, \mathrm{H}-3 \mathrm{eeq}, \mathrm{CH}_{2} \mathrm{CH}_{2} \mathrm{C}(=\mathrm{O}) \mathrm{O}\right), 2.35-2.27\left(\mathrm{~m}, 2 \mathrm{H}, \mathrm{CH}_{2} \mathrm{CH}_{2} \mathrm{C}(=\mathrm{O}) \mathrm{O}\right), 2.15-1.89(\mathrm{~m}, 28 \mathrm{H}$, $\left.9 \mathrm{C}(=\mathrm{O}) \mathrm{CH}_{3}, \mathrm{H}-3 \mathrm{e} a x\right), 0.88-0.69$ (m, $\left.2 \mathrm{H}, \mathrm{OCH}_{2} \mathrm{CH}_{2} \mathrm{SiMe}_{3}\right),-0.16$ (s, $\left.9 \mathrm{H}, \mathrm{OCH}_{2} \mathrm{CH}_{2} \mathrm{SiMe}_{3}\right) ;{ }^{13} \mathrm{C}(125$ $\left.\mathrm{MHz}, \mathrm{CDCl}_{3}\right) \delta 206.1,171.5,170.9,170.7,170.5,170.3,170.1,169.9,169.7,167.8,165.3,165.1$, $138.9,138.2,138.1,133.4,133.3,133.2,129.8,129.7,129.6,129.1,128.6,128.5,128.5,128.4,128.3$, $127.9,127.9,127.8,127.7,127.6,127.5,127.4,100.6,100.2,99.9,99.7,99.0,78.0,77.4,74.6,73.6$, $73.4,73.3,73.2,73.1,72.7,71.8,71.8,71.6,71.5,70.2,70.1,69.7,68.8,68.8,68.1,67.6,67.4,67.2$, 62.7, 62.5, 57.6, 52.9, 49.5, 37.8, 37.7, 29.7, 27.9, 23.4, 23.2, 22.7, 21.0, 20.8, 20.7, 20.6, 17.8, -1.5. $\mathrm{m} / \mathrm{z}$ (MALDI): found $[\mathrm{M}+\mathrm{Na}]^{+} 2198.83, \mathrm{C}_{111} \mathrm{H}_{133} \mathrm{~N}_{3} \mathrm{O}_{40} \mathrm{Si}$ calcd for $[\mathrm{M}+\mathrm{Na}]^{+} 2198.81$. 


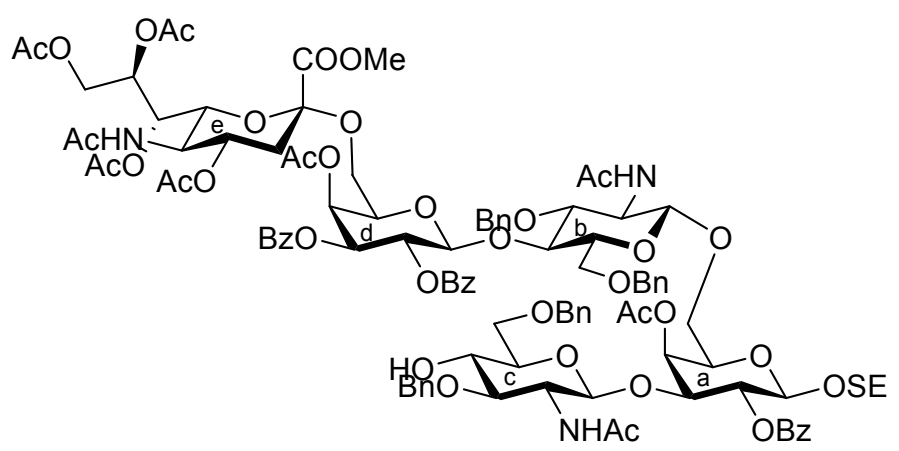

2-(Trimethylsilyl)ethyl (methyl 5-acetamido-4,7,8,9-tetra-O-acetyl-3,5-dideoxy-D-glycero- $\alpha$-D-galacto-2nonulopyranosylonate)-(2 $\rightarrow 6)-4-O$-acetyl-2,3-di-O-benzoyl- $\beta$-D-galactopyranosyl- $(1 \rightarrow 4)$-2-acetamido3,6-di-O-benzyl-2-deoxy- $\beta$-D-glucopyranosyl- $(1 \rightarrow 6)$-[2-acetamido-3,6-di-O-benzyl-2-deoxy- $\beta$ - $D$ -

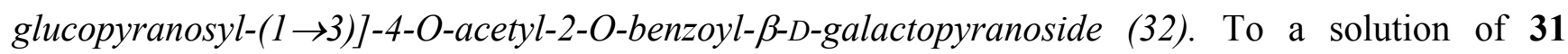
(119 mg, $54.7 \mu \mathrm{mol})$ in THF $(2.0 \mathrm{~mL})$ was added hydrazine monoacetate $(4.9 \mathrm{mg}, 54.7 \mu \mathrm{mol})$ at $0{ }^{\circ} \mathrm{C}$. After stirring for $1 \mathrm{~h}$ at r.t. as the reaction was monitored by TLC (30:1 $\left.\mathrm{CHCl}_{3}-\mathrm{MeOH}\right)$, THF was evaporated. The residue was diluted with $\mathrm{CHCl}_{3}$ and washed with $2 \mathrm{M} \mathrm{HCl}$, satd aq $\mathrm{NaHCO}_{3}$, and brine, dried over $\mathrm{Na}_{2} \mathrm{SO}_{4}$, and concentrated. The obtained residue was purified by silica gel column chromatography $\left(40: 1 \mathrm{CHCl}_{3}-\mathrm{MeOH}\right)$ to give $32(104 \mathrm{mg}, 92 \%)$. $[\alpha]_{\mathrm{D}}-5.2^{\circ}\left(\mathrm{c} 1.9, \mathrm{CHCl}_{3}\right) ;{ }^{1} \mathrm{H}-\mathrm{NMR}$ $\left(500 \mathrm{MHz}, \mathrm{CDCl}_{3}\right) \delta 8.02-7.20(\mathrm{~m}, 35 \mathrm{H}, 7 \mathrm{Ph}), 6.01\left(\mathrm{~d}, 1 \mathrm{H}, J_{\mathrm{NH}, 2}=8.6 \mathrm{~Hz}, \mathrm{NHb}\right), 5.64(\mathrm{~d}, 1 \mathrm{H}$, $J_{3,4}=3.5 \mathrm{~Hz}, \mathrm{H}-4 \mathrm{~d}$ ), 5.58 (near t, $1 \mathrm{H}, J_{1,2}=8.0 \mathrm{~Hz}, J_{2,3}=10.3 \mathrm{~Hz}, \mathrm{H}-2 \mathrm{~d}$ ), 5.43-5.32 (m, $6 \mathrm{H}, \mathrm{H}-2 \mathrm{a}, \mathrm{H}-4 \mathrm{a}$, H-3d, H-7e, H-8e, NHc), 5.21 (d, 1 H, $J_{\mathrm{NH}, 5}=7.4 \mathrm{~Hz}, \mathrm{NHe}$ ), 4.89-4.81 (m, 3 H, H-1d, H-4e, $\mathrm{OCH}_{2}$ ), $4.82\left(\mathrm{~d}, 1 \mathrm{H}, J_{1,2}=9.7 \mathrm{~Hz}, \mathrm{H}-1 \mathrm{c}\right), 4.72-4.65\left(2 \mathrm{~d}, 2 \mathrm{H}, J_{\text {gem }}=11.5 \mathrm{~Hz}, 2 \mathrm{OCH}_{2}\right), 4.60-4.45(\mathrm{~m}, 5 \mathrm{H}$, $\left.\mathrm{H}-1 \mathrm{~b}, 4 \mathrm{OCH}_{2}\right), 4.44$ (d, $\left.1 \mathrm{H}, J_{1,2}=8.0 \mathrm{~Hz}, \mathrm{H}-1 \mathrm{a}\right), 4.37-4.32\left(\mathrm{~m}, 2 \mathrm{H}, \mathrm{OCH}_{2}, \mathrm{H}-9 \mathrm{e}\right), 4.12-4.04$ (m, $4 \mathrm{H}$, H-6a, H-6d, H-6e, H-9'e), 3.98-3.62 (m, 14 H, H-3a, H-5a, H-6'a, H-2b, H-3b, H-6b, H-6'b, H-3c, H-5d, H-6'd, $\left.\mathrm{OCH}_{3}, \mathrm{OCH}_{2} \mathrm{CH}_{2} \mathrm{SiMe}_{3}\right), 3.54$ (t, $\left.1 \mathrm{H}, J_{3,4}=J_{4,5}=12.0 \mathrm{~Hz}, \mathrm{H}-4 \mathrm{c}\right), 3.49-3.36(\mathrm{~m}, 6 \mathrm{H}, \mathrm{H}-5 \mathrm{c}$, H-6c, H-6'c, H-4b, H-5b, OCH $\mathrm{CH}_{2} \mathrm{SiMe}_{3}$ ), 3.16 (dd, 1 H, H-2c), 2.96 (s, $1 \mathrm{H}, \mathrm{OH}$ ), 2.53 (dd, $1 \mathrm{H}$, $\left.J_{\text {gem }}=12.4 \mathrm{~Hz}, J_{3 \mathrm{eq}, 4}=4.6 \mathrm{~Hz}, \mathrm{H}-3 \mathrm{e} e q\right), 2.18-1.87\left(\mathrm{~m}, 25 \mathrm{H}, 8 \mathrm{C}(=\mathrm{O}) \mathrm{CH}_{3}, \mathrm{H}-3 \mathrm{e} a x\right), 0.88-0.69$ (m, $\left.2 \mathrm{H}, \mathrm{OCH}_{2} \mathrm{CH}_{2} \mathrm{SiMe}_{3}\right),-0.16$ (s, $\left.9 \mathrm{H}, \mathrm{OCH}_{2} \mathrm{CH}_{2} \mathrm{SiMe}_{3}\right) ;{ }^{13} \mathrm{C}-\mathrm{NMR}\left(125 \mathrm{MHz}, \mathrm{CDCl}_{3}\right) \delta 170.8,170.6$, $170.4,170.2,170.1,169.9,169.6,169.5,167.7,165.6,165.2,165.0,138.7,138.4,138.0,137.7,133.4$, 133.2 , 133.1, 129.7, 129.7, 129.6, 129.5, 129.0, 128.4, 128.4, 128.3, 128.3, 128.2, 127.8, 127.8, 127.7, $127.6,127.5,127.3,100.4,100.2,100.1,99.6,98.9,79.8,77.9,75.2,74.5,73.8,73.5,73.5,73.2,73.1$, 73.0, 72.8, 72.6, 71.7, 71.6, 71.4, 70.7, 70.1, 70.0, 68.7, 68.1, 67.5, 67.3, 67.2, 62.5, 62.4, 56.4, 54.0, $52.8,49.2,37.7,29.6,23.3,23.1,22.8,20.9,20.7,20.6,20.5,17.7,-1.6 . \mathrm{m} / z$ (MALDI): found $[\mathrm{M}+\mathrm{Na}]^{+} 2100.96, \mathrm{C}_{106} \mathrm{H}_{127} \mathrm{~N}_{3} \mathrm{O}_{38} \mathrm{Si}$ calcd for $[\mathrm{M}+\mathrm{Na}]^{+} 2100.78$.

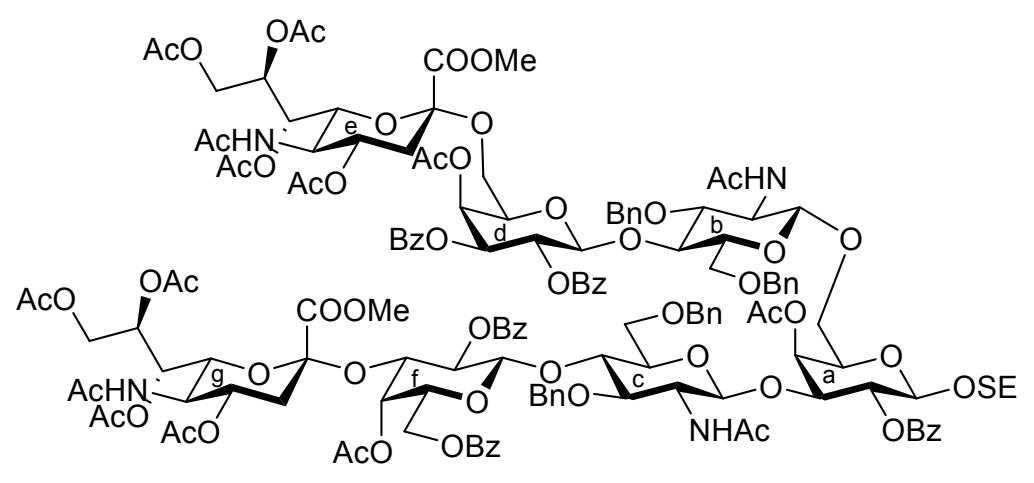


2-(Trimethylsilyl)ethyl (methyl 5-acetamido-4,7,8,9-tetra-O-acetyl-3,5-dideoxy-D-glycero- $\alpha$-D-galacto-2nonulopyranosylonate)-(2 $\rightarrow 3)-4-O$-acetyl-2,6-di-O-benzoyl- $\beta$-D-galactopyranosyl- $(1 \rightarrow 4)$-2-acetamido3,6-di-O-benzyl-2-deoxy- $\beta$-D-glucopyranosyl-(1 $\rightarrow 3)$-[(methyl 5-acetamido-4, 7,8,9-tetra-O-acetyl-3,5dideoxy-D-glycero- $\alpha$-D-galacto-2-nonulopyranosylonate)-(2 $\rightarrow 6)-4$-O-acetyl-2,3-di-O-benzoyl- $\beta$ - $D$ galactopyranosyl-( $1 \rightarrow 4)$-2-acetamido-3,6-di-O-benzyl-2-deoxy- $\beta$-D-glucopyranosyl-( $1 \rightarrow 6)]$-4O-acetyl-2-O-benzoyl- $\beta$-D-galactopyranoside (33). To a mixture of 4 (103 $\mathrm{mg}, 98.7 \mu \mathrm{mol})$ and 32 (93 mg, $44.5 \mu \mathrm{mol})$ in $\mathrm{CH}_{2} \mathrm{Cl}_{2}(1.8 \mathrm{~mL})$ was added $3 \AA$ molecular sieves $(300 \mathrm{mg})$ at r.t. After stirring for $1 \mathrm{~h}$, the mixture was cooled to $0{ }^{\circ} \mathrm{C}$. TMSOTf $(1.8 \mu \mathrm{L}, 9.94 \mu \mathrm{mol})$ was then added to the mixture at $0{ }^{\circ} \mathrm{C}$. The reaction was stirred for $2 \mathrm{~h}$ at r.t. as the reaction was monitored by TLC $\left(15: 1: 1 \mathrm{CHCl}_{3}-\right.$ $\mathrm{MeOH}-\mathrm{EtOAc})$. Another portion of TMSOTf $(1.8 \mu \mathrm{L}, 9.94 \mu \mathrm{mol})$ was added to the mixture at $0{ }^{\circ} \mathrm{C}$. After the stirring was continued for $4 \mathrm{~h}$ at r.t, the reaction was quenched by the addition of triethylamine. The reaction mixture was diluted with $\mathrm{CHCl}_{3}$ and filtered through Celite. The filtrate was then washed with satd aq $\mathrm{NaHCO}_{3}$ and brine. The organic layer was subsequently dried over $\mathrm{Na}_{2} \mathrm{SO}_{4}$, and concentrated. The resulting residue was purified by silica gel column chromatography $\left(40: 1 \mathrm{CHCl}_{3}-\mathrm{MeOH}\right)$ to give $33(82 \mathrm{mg}, 62 \%)$. [ $\left.\alpha\right]_{\mathrm{D}}-5.1^{\circ}$ (c $\left.0.8, \mathrm{CHCl}_{3}\right) ;{ }^{1} \mathrm{H}-\mathrm{NMR}(600 \mathrm{MHz}$, $\left.\mathrm{CDCl}_{3}\right) \delta 8.22-7.12(\mathrm{~m}, 45 \mathrm{H}, 9 \mathrm{Ph}), 5.92(\mathrm{~d}, 1 \mathrm{H}, \mathrm{NHb}), 5.65(\mathrm{~m}, 1 \mathrm{H}, \mathrm{H}-4 \mathrm{~g}), 5.63\left(\mathrm{~d}, 1 \mathrm{H}, J_{3,4}=3.4 \mathrm{~Hz}\right.$, $\mathrm{H}-4 \mathrm{~d}$ ), 5.55 (near t, $1 \mathrm{H}, J_{1,2}=8.2 \mathrm{~Hz}, J_{2,3}=10.2 \mathrm{~Hz}, \mathrm{H}-2 \mathrm{~d}$ ), 5.40 (m, $\left.1 \mathrm{H}, \mathrm{H}-8 \mathrm{e}\right), 5.37$ (dd, $1 \mathrm{H}, \mathrm{H}-3 \mathrm{~d}$ ), 5.33-5.13 (m, 7 H, H-2a, H-4a, H-7e, H-2f, H-7g, NHc, NHe), 5.06 (d, 1 H, $J_{3,4}=3.4$ Hz, H-4f), 5.01 $\left(\mathrm{d}, 1 \mathrm{H}, J_{1,2}=7.5 \mathrm{~Hz}, \mathrm{H}-1 \mathrm{f}\right), 5.00\left(\mathrm{~d}, 1 \mathrm{H}, J_{5, \mathrm{NH}}=7.5 \mathrm{~Hz}, \mathrm{NHg}\right), 4.87-4.82$ (m, $5 \mathrm{H}, \mathrm{H}-1 \mathrm{~d}, \mathrm{H}-4 \mathrm{e}, \mathrm{H}-4 \mathrm{~g}$, $\left.2 \mathrm{OCH}_{2}\right), 4.75\left(\mathrm{dd}, 1 \mathrm{H}, J_{2,3}=10.2 \mathrm{~Hz}, \mathrm{H}-3 \mathrm{f}\right), 4.71\left(\mathrm{~d}, 1 \mathrm{H}, J_{\text {gem }}=11.6 \mathrm{~Hz}, \mathrm{OCH}_{2}\right), 4.68(\mathrm{~d}, 1 \mathrm{H}$, $\left.J_{1,2}=7.5 \mathrm{~Hz}, \mathrm{H}-1 \mathrm{c}\right), 4.68-4.47$ (m, $\left.4 \mathrm{H}, \mathrm{H}-1 \mathrm{~b}, 3 \mathrm{OCH}_{2}\right), 4.42$ (d, $\left.1 \mathrm{H}, J_{1,2}=10.7 \mathrm{~Hz}, \mathrm{H}-1 \mathrm{a}\right), 4.28(\mathrm{~m}, 4 \mathrm{H}$, H-9e, H-9g, $2 \mathrm{OCH}_{2}$ ), 4.11-4.00 (m, 7 H, H-4b, H-4c, H-6c, H-6'c, H-5e, H-6e, H-9'e), 3.97-3.55 (m, 23 H, H-3a, H-5a, H-6a, H-6'a, H-2b, H-3b, H-6b, H-6'b, H-3c, H-6d, H-6'd, H-6f, H-6'f, H-5g, H-6g, H-9'g, $2 \mathrm{OCH}_{3}, \mathrm{OCH}_{2} \mathrm{CH}_{2} \mathrm{SiMe}_{3}$ ), 3.49-3.40 (m, $3 \mathrm{H}, \mathrm{H}-5 \mathrm{~b}, \mathrm{H}-5 \mathrm{c}, \mathrm{OCH}_{2} \mathrm{CH}_{2} \mathrm{SiMe}_{3}$ ), 3.31-3.28 (m, $2 \mathrm{H}, \mathrm{H}-5 \mathrm{c}, \mathrm{H}-5 \mathrm{f}$ ), 3.14 (br dd, $1 \mathrm{H}, \mathrm{H}-2 \mathrm{c}$ ), 2.53 (dd, $\left.1 \mathrm{H}, J_{\text {gem }}=11.3 \mathrm{~Hz}, J_{3 \text { eq }, 4}=4.8 \mathrm{~Hz}, \mathrm{H}-3 \mathrm{~g} e q\right), 2.49$ $\left(\mathrm{m}, 1 \mathrm{H}, J_{\mathrm{gem}}=11.7 \mathrm{~Hz}, J_{3 \mathrm{eq}, 4}=4.8 \mathrm{~Hz}, \mathrm{H}-3 \mathrm{e} e q\right), 2.15-1.74(\mathrm{~m}, 43 \mathrm{H}, 14 \mathrm{Ac}, \mathrm{H}-3 \mathrm{e} a x), 1.70(\mathrm{t}, 1 \mathrm{H}$, $\mathrm{H}-3 \mathrm{gax}), 1.51$ (s, $3 \mathrm{H}, \mathrm{Ac}), 0.88-0.68\left(\mathrm{~m}, 2 \mathrm{H}, \mathrm{OCH}_{2} \mathrm{CH}_{2} \mathrm{SiMe}_{3}\right),-0.17$ (s, $9 \mathrm{H}, \mathrm{OCH}_{2} \mathrm{CH}_{2} \mathrm{SiMe}_{3}$ ); ${ }^{13} \mathrm{C}-\mathrm{NMR}\left(150 \mathrm{MHz}, \mathrm{CDCl}_{3}\right) \delta 170.9,170.7,170.7,170.3,170.2,170.1,170.1,170.0,169.8,169.7$, 169.6, 168.0, 167.8, 165.7, 165.5, 165.3, 165.2, 164.9, 138.8, 138.8, 138.7, 138.1, 133.4, 133.3, 133.2, $133.1,133.0,130.3,129.9,129.8,129.7,129.7,129.6,129.5,129.0,128.6,128.5,128.4,128.2,128.1$, $128.0,127.9,129.6,129.5,129.0,128.6,128.5,128.4,128.2,128.1,128.0,127.9,127.8,127.5,127.4$, $127.2,127.2,127.0,100.5,100.3,99.6,99.5,99.0,96.8,78.1,77.6,77.5,75.2,75.0,74.6,74.5,73.6$, $73.3,73.0,72.7,72.6,71.8,71.7,71.4,71.3,70.4,70.2,70.1,69.4,68.7,68.1,68.0,67.5,67.3,67.2$, 67.1, 66.6, 62.4, 61.3, 53.0, 52.9, 49.4, 48.8, 37.8, 37.3, 29.7, 23.3, 23.2, 23.1, 22.6, 21.3, 21.0, 20.8, 20.8, 20.7, 20.6, 20.6, 20.3, 17.7, -1.5. $\mathrm{m} / z$ (MALDI): found $[\mathrm{M}+\mathrm{Na}]^{+} 2986.28, \mathrm{C}_{148} \mathrm{H}_{174} \mathrm{~N}_{4} \mathrm{O}_{58} \mathrm{Si}$ calcd for $[\mathrm{M}+\mathrm{Na}]^{+} 2986.05$. 


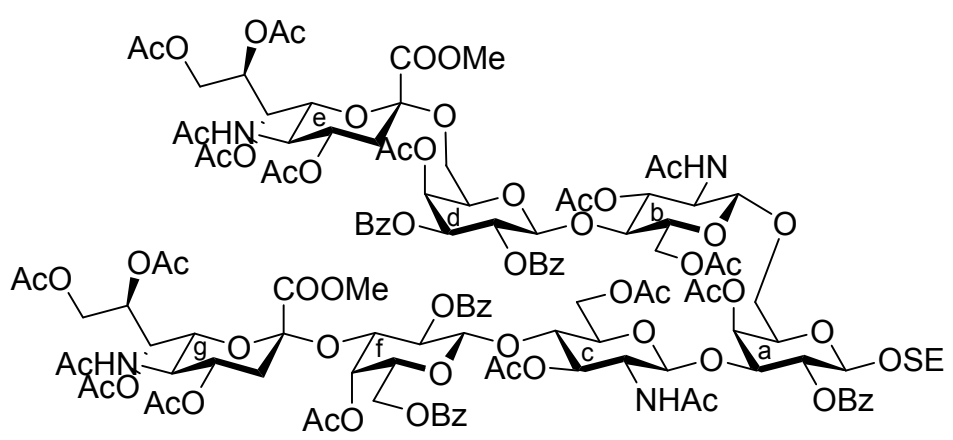

2-(Trimethylsilyl)ethyl (methyl 5-acetamido-4,7,8,9-tetra-O-acetyl-3,5-dideoxy-D-glycero- $\alpha$-D-galacto-2nonulopyranosylonate)-(2 $\rightarrow 3)-4-O$-acetyl-2,6-di-O-benzoyl- $\beta$-D-galactopyranosyl- $(1 \rightarrow 4)$-2-acetamido3,6-di-O-acetyl-2-deoxy- $\beta$-D-glucopyranosyl- $(1 \rightarrow 3)$-[(methyl 5-acetamido-4, 7,8,9-tetra-O-acetyl-3,5dideoxy-D-glycero- $\alpha$-D-galacto-2-nonulopyranosylonate)-(2 $\rightarrow 6)-4$-O-acetyl-2,3-di-O-benzoyl- $\beta$ - $D$ galactopyranosyl- $(1 \rightarrow 4)$-2-acetamido-3,6-di-O-acetyl-2-deoxy- $\beta$-D-glucopyranosyl- $(1 \rightarrow 6)]-4-O$ acetyl-2-O-benzoyl- $\beta$-D-galactopyranoside (35). To a solution of 33 (42.0 $\mathrm{mg}, 14.2 \mu \mathrm{mol})$ in 1,4-dioxane $(0.6 \mathrm{~mL})$ was added $\mathrm{Pd}(\mathrm{OH})_{2} / \mathrm{C}(210 \mathrm{mg})$. After stirring for $4 \mathrm{~h}$ at r.t. under a hydrogen atmosphere as the reaction was monitored by TLC $\left(10: 1 \mathrm{CHCl}_{3}-\mathrm{MeOH}\right)$, the mixture was filtered through Celite. The filtrate was concentrated and the obtained crude residue was roughly purified by silica gel column chromatography $\left(10: 1 \mathrm{CHCl}_{3}-\mathrm{MeOH}\right)$. The obtained product was exposed to high vacuum for $24 \mathrm{~h}$. The residue was then dissolved in pyridine $(1.4 \mathrm{~mL})$. Acetic anhydride $(11 \mu \mathrm{L}$, $0.114 \mathrm{mmol})$ and DMAP $(1.0 \mathrm{mg}, 8.18 \mu \mathrm{mol})$ were added to the mixture at $0{ }^{\circ} \mathrm{C}$. After stirring for $72 \mathrm{~h}$ at r.t. as the reaction was monitored by TLC $\left(10: 1 \mathrm{CHCl}_{3}-\mathrm{MeOH}\right)$, the reaction was quenched by the addition of $\mathrm{MeOH}$ at $0{ }^{\circ} \mathrm{C}$. The mixture was co-evaporated with toluene and the residue was then diluted with $\mathrm{CHCl}_{3}$, and washed with $2 \mathrm{M} \mathrm{HCl}, \mathrm{H}_{2} \mathrm{O}$, satd aq $\mathrm{NaHCO}_{3}$ and brine. The organic layer was subsequently dried over $\mathrm{Na}_{2} \mathrm{SO}_{4}$, and concentrated. The resulting residue was purified by silica gel column chromatography $\left(40: 1 \mathrm{CHCl}_{3}-\mathrm{MeOH}\right)$ to give $35(35 \mathrm{mg}, 89 \%)$. $[\alpha]_{\mathrm{D}}+5.1^{\circ}\left(\mathrm{c} 0.7, \mathrm{CHCl}_{3}\right)$; ${ }^{1} \mathrm{H}-\mathrm{NMR}\left(500 \mathrm{MHz}, \mathrm{CDCl}_{3}\right) \delta 8.17-7.27(\mathrm{~m}, 25 \mathrm{H}, 5 \mathrm{Ph}), 5.92\left(\mathrm{~d}, 1 \mathrm{H}, J_{\mathrm{NH}, 2}=9.2 \mathrm{~Hz}, \mathrm{NHb}\right), 5.68(\mathrm{~d}$, $1 \mathrm{H}, J_{3,4}=3.5 \mathrm{~Hz}, \mathrm{H}-4 \mathrm{~d}$ ), $5.77\left(\mathrm{~m}, 1 \mathrm{H}, \mathrm{H}-8 \mathrm{~g}\right.$ ), 5.55 (near t, $2 \mathrm{H}, J_{1,2}=8.0 \mathrm{~Hz}, J_{2,3}=10.3 \mathrm{~Hz}, \mathrm{H}-2 \mathrm{~d}$ ), 5.44 (m, 1 H, H-3d), 5.38 (m, 1 H, H-8e), 5.32-5.16 (m, 7 H, H-2a, H-3a, H-3b, H-7e, H-2f, H-7g, $\mathrm{NHe}$ ), 5.09 (d, $\left.1 \mathrm{H}, J_{3,4}=2.8 \mathrm{~Hz}, \mathrm{H}-4 \mathrm{f}\right), 4.96$ (d, $\left.1 \mathrm{H}, J_{\mathrm{NH}, 5}=9.8 \mathrm{~Hz}, \mathrm{NHg}\right), 4.91-4.80$ (m, $6 \mathrm{H}, \mathrm{NHc}$, H-3c, H-1d, H-4e, H-1f, H-4g), 4.74-4.71 (dd, 1 H, $J_{2,3}=9.7$ Hz, H-3f), 4.47 (d, 1 H, $J_{1,2}=8.0$ Hz, H-1b), 4.43 (d, 1 H, $J_{1,2}=8.0$ Hz, H-1a), 4.41-4.28 (m, 6 H, H-6a, H-1c, H-6d, H-9e, H-6f, H-9g), 4.17-3.93 (m, 10 H, H-6'a, H-6b, H-6'b, H-6'd, H-5e, H-6e, H-9'e, H-6'f, H-9'g, OCH $\mathrm{CH}_{2} \mathrm{SiMe}_{3}$ ), 3.88-3.68 (m, 15 H, H-3a, H-2b, H-4b, H-5b, H-2c, H-4c, H-6c, H-6'c, H-5g, $2 \mathrm{OCH}_{3}$ ), 3.55-3.53 (m, $2 \mathrm{H}, \mathrm{H}-5 \mathrm{~d}, \mathrm{H}-6 \mathrm{~g}), 3.48-3.42$ (m, $3 \mathrm{H}, \mathrm{H}-5 \mathrm{a}, \mathrm{H}-5 \mathrm{f}, \mathrm{OCH}_{2} \mathrm{CH}_{2} \mathrm{SiMe}_{3}$ ), 3.27-3.25 (m, $1 \mathrm{H}, J_{5,4}=9.7 \mathrm{~Hz}$, $\mathrm{H}-5 \mathrm{c}), 2.55\left(\mathrm{dd}, 1 \mathrm{H}, J_{\text {gem }}=12.6 \mathrm{~Hz}, J_{3 \mathrm{eq}, 4}=4.6 \mathrm{~Hz}, \mathrm{H}-3 \mathrm{geq}\right), 2.51\left(\mathrm{dd}, 1 \mathrm{H}, J_{\text {gem }}=12.6 \mathrm{~Hz}, J_{3 \mathrm{eq}, 4}=4.6 \mathrm{~Hz}\right.$, H-3eeq), 2.20-1.78 (m, 52 H, 17 Ac, H-3eax), 1.62 (t, 1 H, H-3gax), 1.54 (s, 6 H, 2 Ac), 0.88-0.72 (m, $\left.2 \mathrm{H}, \mathrm{OCH}_{2} \mathrm{CH}_{2} \mathrm{SiMe}_{3}\right),-0.15$ (s, $9 \mathrm{H}, \mathrm{OCH}_{2} \mathrm{CH}_{2} \mathrm{SiMe}$ ); ${ }^{13} \mathrm{C}-\mathrm{NMR}\left(150 \mathrm{MHz}, \mathrm{CDCl}_{3}\right) \delta 170.9,170.7$, $170.7,170.5,170.4,170.4,170.2,170.1,170.1,170.0,169.8,169.8,169.7,168.0,167.7,165.6,165.3$, $165.0,164.9,164.8,133.5,133.3,133.2,130.3,129.8,129.7,129.5,129.0,128.9,128.7,128.5,128.5$, $128.4,128.4,101.2,100.7,100.6,100.5,100.4,99.2,96.8,75.6,74.6,72.9,72.7,72.5,72.4,71.9$, $71.7,71.1,70.9,70.0,69.8,69.6,69.4,68.6,67.8,67.4,67.1,66.2,62.9,62.4,62.2,62.0,61.0,60.9$, 53.9, 53.0, 49.4, 48.8, 37.8, 37.2, 23.2, 23.1, 22.7, 21.3, 21.0, 20.9, 20.8, 20.7, 20.6, 20.5, 20.4, 17.8, -1.5. $\mathrm{m} / z$ (MALDI): found $[\mathrm{M}+\mathrm{Na}]^{+} 2793.65, \mathrm{C}_{128} \mathrm{H}_{158} \mathrm{~N}_{4} \mathrm{O}_{62} \mathrm{Si}$ calcd for $[\mathrm{M}+\mathrm{Na}]^{+} 2793.90$. 


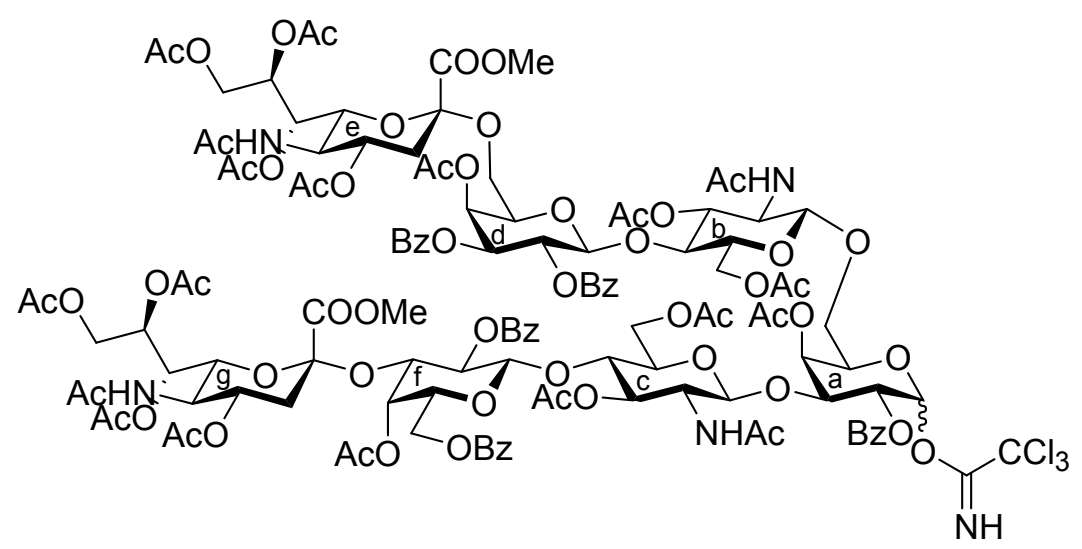

(Methyl 5-acetamido-4,7,8,9-tetra-O-acetyl-3,5-dideoxy-D-glycero- $\alpha$-D-galacto-2-nonulopyranosylonate)$(2 \rightarrow 3)$-4-O-acetyl-2,6-di-O-benzoyl- $\beta$-D-galactopyranosyl-( $1 \rightarrow 4)$-2-acetamido-3, 6-di-O-acetyl-2deoxy- $\beta$-D-glucopyranosyl- $(1 \rightarrow 3)$-[(methyl 5-acetamido-4, 7,8,9-tetra-O-acetyl-3,5-dideoxy-D-glycero$\alpha$-D-galacto-2-nonulopyranosylonate)-(2 $\rightarrow 6)$-4-O-acetyl-2,3-di-O-benzoyl- $\beta$-D-galactopyranosyl$(1 \rightarrow 4)$-2-acetamido-3, 6-di-O-acetyl-2-deoxy- $\beta$-D-glucopyranosyl- $(1 \rightarrow 6)]$-4-O-acetyl-2-O-benzoyl-Dgalactopyranosyl trichloroacetimidate (37). To a solution of 35 (28 $\mathrm{mg}, 10.2 \mu \mathrm{mol})$ in $\mathrm{CH}_{2} \mathrm{Cl}_{2}$ $(0.75 \mathrm{~mL})$ was added TFA $(0.25 \mathrm{~mL})$ at $0{ }^{\circ} \mathrm{C}$. After stirring for $2 \mathrm{~h}$ at $0{ }^{\circ} \mathrm{C}$ as the reaction was monitored by TLC (15:1:1 $\left.\mathrm{CHCl}_{3}-\mathrm{MeOH}-\mathrm{EtOAc}\right)$, the reaction mixture was co-evaporated with toluene and then roughly purified by silica gel column chromatography $\left(20: 1 \mathrm{CHCl}_{3}-\mathrm{MeOH}\right)$. The obtained product was exposed to high vacuum for $24 \mathrm{~h}$ and then dissolved in $\mathrm{CH}_{2} \mathrm{Cl}_{2}(1.0 \mathrm{~mL})$. $\mathrm{CCl}_{3} \mathrm{CN}(10.2 \mu \mathrm{L}, 0.102 \mathrm{mmol})$ and DBU $(1.8 \mu \mathrm{L}, 12.2 \mu \mathrm{mol})$ were added to the mixture at $0{ }^{\circ} \mathrm{C}$. After stirring for $45 \mathrm{~min}$ at $0{ }^{\circ} \mathrm{C}$ as the reaction was monitored by TLC $\left(10: 1 \mathrm{CHCl}_{3}-\mathrm{MeOH}\right)$, the reaction mixture was evaporated. The obtained crude residue was purified by silica gel column chromatography (30:1 $\left.\mathrm{CHCl}_{3}-\mathrm{MeOH}\right)$ to give 37 (27 mg, 95\%, $\left.\alpha: \beta=3: 1\right) .37 \alpha:{ }^{1} \mathrm{H}-\mathrm{NMR}(600 \mathrm{MHz}$, $\left.\mathrm{CDCl}_{3}\right) \delta 8.60(\mathrm{~s}, 1 \mathrm{H}, \mathrm{C}(=\mathrm{NH})), 8.19-7.27(\mathrm{~m}, 25 \mathrm{H}, 5 \mathrm{Ph}), 6.48\left(\mathrm{~d}, 1 \mathrm{H}, J_{1,2}=4.1 \mathrm{~Hz}, \mathrm{H}-1 \mathrm{a}\right), 5.81(\mathrm{~d}$, $\left.1 \mathrm{H}, J_{\mathrm{NH}, 2}=9.0 \mathrm{~Hz}, \mathrm{NHc}\right), 5.68\left(\mathrm{~d}, 1 \mathrm{H}, J_{3,4}=3.5 \mathrm{~Hz}, \mathrm{H}-4 \mathrm{~d}\right), 5.60(\mathrm{~m}, 1 \mathrm{H}, \mathrm{H}-8 \mathrm{~g}), 5.55$ (near t, $1 \mathrm{H}$, $\left.J_{1,2}=8.3 \mathrm{~Hz}, J_{2,3}=10.3 \mathrm{~Hz}, \mathrm{H}-2 \mathrm{~d}\right), 5.48$ (dd, $\left.1 \mathrm{H}, J_{2,3}=10.3 \mathrm{~Hz}, \mathrm{H}-2 \mathrm{a}\right), 5.43-5.42$ (m, $\left.2 \mathrm{H}, \mathrm{H}-3 \mathrm{a}, \mathrm{H}-4 \mathrm{a}\right)$, 5.38 (m, $1 \mathrm{H}, \mathrm{H}-8 \mathrm{e}), 5.32$ (dd, $1 \mathrm{H}, \mathrm{H}-7 \mathrm{e}), 5.24$ (m, $2 \mathrm{H}, \mathrm{H}-3 \mathrm{a}, \mathrm{H}-7 \mathrm{~g}), 5.20$ (near t, $1 \mathrm{H}, J_{1,2}=8.3 \mathrm{~Hz}$, $\left.J_{2,3}=9.6 \mathrm{~Hz}, \mathrm{H}-2 \mathrm{f}\right), 5.16\left(\mathrm{t}, 1 \mathrm{H}, J_{2,3}=J_{3,4}=9.0 \mathrm{~Hz}, \mathrm{H}-3 \mathrm{~b}\right), 5.13\left(\mathrm{~d}, 1 \mathrm{H}, J_{5, \mathrm{NH}}=9.6 \mathrm{~Hz}, \mathrm{NHe}\right), 4.92(\mathrm{~d}$, $1 \mathrm{H}, J_{3,4}=2.3 \mathrm{~Hz}, \mathrm{H}-4 \mathrm{f}$ ), 4.90-4.80 (m, 8 H, H-3c, H-1d, H-4e, H-1f, H-3d, H-4g, NHc, NHg), 4.73 (dd, $1 \mathrm{H}, \mathrm{H}-3 \mathrm{f}), 4.55$ (d, $\left.1 \mathrm{H}, J_{1,2}=7.6 \mathrm{~Hz}, \mathrm{H}-1 \mathrm{~b}\right), 4.45$ (d, $\left.1 \mathrm{H}, J_{1,2}=7.4 \mathrm{~Hz}, \mathrm{H}-1 \mathrm{c}\right), 4.41-4.01$ (m, $13 \mathrm{H}$, H-3a, H-6a, H-6'a, H-5e, H-6e, H-9e, H-9'e, H-6d, H-6'd, H-6f, H-6'f, H-9g, H-9'g), 3.88-3.70 (m, 15 H, H-2b, H-4b, H-6b, H-6'b, H-2c, H-4c, H-6c, H-6'c, H-5e, $2 \mathrm{OCH}_{3}$ ), 3.55-3.36 (m, 5 H, H-5a, H-5b, H-5c, H-5d, H-5f), 2.55 (dd, $\left.1 \mathrm{H}, J_{\text {gem }}=13.1 \mathrm{~Hz}, J_{3 \mathrm{eq}, 4}=4.8 \mathrm{~Hz}, \mathrm{H}-3 \mathrm{e} e q\right), 2.51$ (dd, $1 \mathrm{H}, J_{\text {gem }}=11.6 \mathrm{~Hz}$, $\left.J_{3 \mathrm{eq}, 4}=4.8 \mathrm{~Hz}, \mathrm{H}-3 \mathrm{geq}\right), 2.20-1.78$ (m, 52 H, 17 Ac, H-3eax), 1.63-1.39 (m, 7 H, 2 Ac, H-3gax).

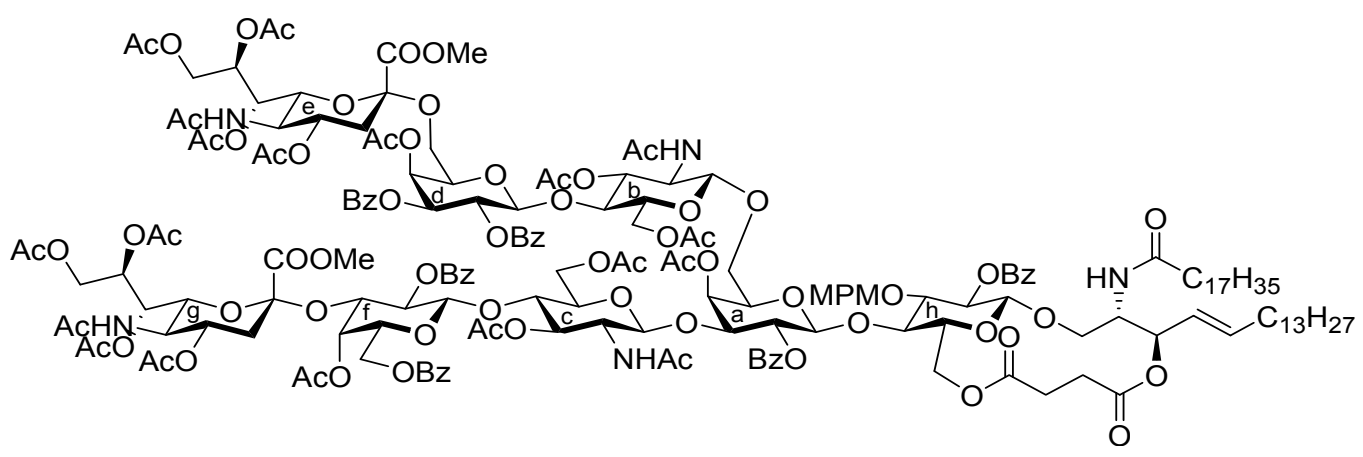


(2S,3R,4E)-1-O-(\{(Methyl 5-acetamido-4, 7,8,9-tetra-O-acetyl-3,5-dideoxy-D-glycero- $\alpha$-D-galacto-2nonulopyranosylonate)-(2 $\rightarrow 3)-4-O$-acetyl-2,6-di-O-benzoyl- $\beta$-D-galactopyranosyl- $(1 \rightarrow 4)$-2-acetamido3,6-di-O-acetyl-2-deoxy- $\beta$-D-glucopyranosyl- $(1 \rightarrow 3)$-[(methyl 5-acetamido-4, 7,8,9-tetra-O-acetyl-3,5dideoxy-D-glycero- $\alpha$-D-galacto-2-nonulopyranosylonate)-(2 $\rightarrow 6)-4-O$-acetyl-2,3-di-O-benzoyl- $\beta$ - $D$ galactopyranosyl-( $(\rightarrow 4)$-2-acetamido-3,6-di-O-acetyl-2-deoxy- $\beta$-D-glucopyranosyl- $(1 \rightarrow 6)]$-4-O-acetyl2-O-benzoyl- $\beta$-D-galactopyranosyl $\}$ - $(1 \rightarrow 4)$-2-O-benzoyl-3-O-p-methoxybenzyl- $\beta$-D-glucopyranosyl)2-octadecanamido-3,6'-succinyl-octadec-4-ene-1,3-diol (39). To a mixture of 37 (38 mg, $13.4 \mu \mathrm{mol})$ and $38(14 \mathrm{mg}, 13.4 \mu \mathrm{mol})$ in $\mathrm{CH}_{2} \mathrm{Cl}_{2}(1.3 \mathrm{~mL})$ was added $5 \AA$ molecular sieves $(100 \mathrm{mg})$ at r.t. After stirring for $1 \mathrm{~h}$, the mixture was cooled to $0{ }^{\circ} \mathrm{C}$. TMSOTf $(0.3 \mu \mathrm{L}, 1.34 \mu \mathrm{mol})$ was then added to the mixture at $0{ }^{\circ} \mathrm{C}$. After stirring for $1 \mathrm{~h}$ at r.t. as the reaction was monitored by TLC $\left(15: 1: 5 \mathrm{CHCl}_{3}-\right.$ $\mathrm{MeOH}-\mathrm{EtOAc}$ ), the reaction was quenched by the addition of triethylamine. The reaction mixture was diluted with $\mathrm{CHCl}_{3}$ and filtered through Celite. The filtrate was then washed with satd aq $\mathrm{NaHCO}_{3}$ and brine. The organic layer was subsequently dried over $\mathrm{Na}_{2} \mathrm{SO}_{4}$, and concentrated. The residue was purified by silica gel column chromatography $\left(40: 1: 5 \mathrm{CHCl}_{3}-\mathrm{MeOH}-\mathrm{EtOAc}\right)$ to give 39 (24 mg, 49\%). $[\alpha]_{\mathrm{D}}-10.3^{\circ}$ (c 2.0, $\mathrm{CHCl}_{3}$ ); ${ }^{1} \mathrm{H}-\mathrm{NMR}\left(500 \mathrm{MHz}, \mathrm{CD}_{3} \mathrm{CN}\right) \delta 8.13-7.39$ (m, $\left.30 \mathrm{H}, 6 \mathrm{Ph}\right), 7.04(\mathrm{~d}$, $2 \mathrm{H}, \mathrm{Ar}), 6.66(\mathrm{~d}, 2 \mathrm{H}, \mathrm{Ar}), 6.14\left(\mathrm{~d}, 1 \mathrm{H}, J_{5, \mathrm{NH}}=9.7 \mathrm{~Hz}, \mathrm{NHe}\right), 6.09\left(\mathrm{~d}, 1 \mathrm{H}, J_{2, \mathrm{NH}}=9.2 \mathrm{~Hz}, \mathrm{NH}^{C e r}\right.$ ), $6.00\left(\mathrm{~d}, 1 \mathrm{H}, J_{5, \mathrm{NH}}=9.7 \mathrm{~Hz}, \mathrm{NHg}\right), 5.95\left(\mathrm{~d}, 1 \mathrm{H}, J_{2, \mathrm{NH}}=9.1 \mathrm{~Hz}, \mathrm{NHb}\right), 5.79\left(\mathrm{~d}, 1 \mathrm{H}, J_{2, \mathrm{NH}}=9.8 \mathrm{~Hz}\right.$, $\mathrm{NHc}$ ), $5.63\left(\mathrm{dt}, 1 \mathrm{H}, J_{5,6}=6.9 \mathrm{~Hz}, J=14.9 \mathrm{~Hz}, J=6.9 \mathrm{~Hz}, \mathrm{H}-5^{\mathrm{Cer}}\right), 5.54$ (d, $\left.1 \mathrm{H}, J_{3,4}=3.5 \mathrm{~Hz}, \mathrm{H}-4 \mathrm{~d}\right)$, 5.47 (m, $3 \mathrm{H}, \mathrm{H}-3 \mathrm{~d}, \mathrm{H}-7 \mathrm{e}, \mathrm{H}-8 \mathrm{e}$ ), 5.37 (near t, $1 \mathrm{H}, J_{1,2}=7.5 \mathrm{~Hz}, J_{2,3}=10.3 \mathrm{~Hz}, \mathrm{H}-2 \mathrm{~d}$ ), $5.36(\mathrm{~m}, 1 \mathrm{H}$, H-8g), 5.27 (d, $\left.1 \mathrm{H}, J_{3,4}=3.5 \mathrm{~Hz}, \mathrm{H}-4 \mathrm{a}\right), 5.25-5.17$ (m, 4 H, H-2a, H-4f, H-7g, H-4 ${ }^{C e r}$ ), 5.11 (br t, $1 \mathrm{H}, J_{3,4}=J_{2,3}=7.5 \mathrm{~Hz}, \mathrm{H}-3^{\mathrm{Cer}}$ ), 5.03 (near t, $1 \mathrm{H}, J_{1,2}=8.0 \mathrm{~Hz}, J_{2,3}=10.3 \mathrm{~Hz}, \mathrm{H}-2 \mathrm{f}$ ), $4.94(\mathrm{t}, 1 \mathrm{H}$, $\left.J_{1,2}=J_{2,3}=8.3 \mathrm{~Hz}, \mathrm{H}-2 \mathrm{~h}\right), 4.88\left(\mathrm{t}, 1 \mathrm{H}, J_{2,3}=J_{3,4}=9.3 \mathrm{~Hz}, \mathrm{H}-3 \mathrm{~b}\right), 4.76(\mathrm{~m}, 8 \mathrm{H}, \mathrm{H}-1 \mathrm{c}, \mathrm{H}-3 \mathrm{c}, \mathrm{H}-1 \mathrm{~d}$, H-4e, H-1f, H-3f, H-4g, $\mathrm{ArCH}_{2}$ ), 4.68 (d, $\left.1 \mathrm{H}, J_{1,2}=8.1 \mathrm{~Hz}, \mathrm{H}-1 \mathrm{a}\right), 4.54$ (d, $1 \mathrm{H}, J_{1,2}=8.5 \mathrm{~Hz}, \mathrm{H}-1 \mathrm{~b}$ ), $4.47(\mathrm{~d}, 1 \mathrm{H}, \mathrm{H}-1 \mathrm{~h}), 4.46\left(\mathrm{~d}, 1 \mathrm{H}, J_{\text {gem }}=10.9 \mathrm{~Hz}, \mathrm{ArCH}_{2}\right), 4.35\left(\mathrm{dd}, 1 \mathrm{H}, J_{8,9}=2.9 \mathrm{~Hz}, J_{\text {gem }}=12.6 \mathrm{~Hz}\right.$, H-9e), 4.30-4.21 (m, 4 H, H-6b, H-6'b, H-6d, H-9g), 4.11-3.89 (m, 13 H, H-3a, H-6'a, H-6c, H-6'c, H-6'd, H-5e, H-6e, H-9'e, H-6f, H-9g, H-6h, H-6'h, H-2 ${ }^{\text {Cer }}$ ), 3.78-3.53 (m, 21 H, H-5a, H-4b, H-2c, H-4c, H-5d, H-6'a, H-6'f, H-5g, H-6g, H-3h, H-4h, H-1 ${ }^{C e r}, 3 \mathrm{OCH}_{3}$ ), 3.51 (m, 1 H, H-2b), 3.40-3.32 (m, 3 H, H-5b, H-5h, H-1 ${ }^{\text {Cer }}$ ), 3.18-3.12 (m, 2 H, H-5c, H-5f), 2.56 (dd, $1 \mathrm{H}, J_{3 \mathrm{eq}, 4}=4.6 \mathrm{~Hz}, J_{\text {gem }}=12.6 \mathrm{~Hz}$, H-3eeq), 2.48-2.34 (m, 5 H, H-3geq, $2 \mathrm{C}(=\mathrm{O}) \mathrm{CH}_{2}$ ), 2.21-1.61 (m, $62 \mathrm{H}, \mathrm{H}-3 \mathrm{eax}, \mathrm{H}-6^{\mathrm{Cer}}, \mathrm{H}^{\mathrm{C}}{ }^{\mathrm{Cer}}$, $\left.\mathrm{C}(=\mathrm{O}) \mathrm{CH}_{2}{ }^{\mathrm{Cer}}, 19 \mathrm{Ac}\right), 1.55-1.08\left(\mathrm{~m}, 53 \mathrm{H}, \mathrm{H}-3 \mathrm{gax}, 26-\mathrm{CH}_{2}-\right), 0.87$ (t, $\left.6 \mathrm{H}, 2-\mathrm{CH}_{3}{ }^{\mathrm{Cer}}\right) ;{ }^{13} \mathrm{C}-\mathrm{NMR}$ $\left(150 \mathrm{MHz}, \mathrm{CDCl}_{3}\right) \delta 172.6,171.0,170.9,170.8,170.7,170.6,170.5,170.4,170.3,170.2,170.1$, $170.0,169.7,169.7,169.5,168.0,167.7,165.6,165.2,165.2,165.1,165.0,164.5,159.1,138.6,133.8$, $133.3,133.1,130.7,130.3,129.8,129.7,129.6,129.5,129.5,129.5,129.0,128.9,128.8,128.6,128.6$, $128.5,128.4,128.3,124.8,113.9,101.6,101.3,100.8,100.6,100.3,99.7,99.3,96.8,91.8,80.8,79.7$, $76.3,75.9,74.7,74.3,73.2,72.9,72.7,72.6,72.5,71.9,71.8,71.7,71.7,71.0,70.9,70.1,69.8,69.7$, $69.4,68.6,67.8,67.4,67.1,66.3,63.2,63.0,62.6,62.1,61.9,61.1,60.9,55.1,53.6,53.1,53.0,49.9$, $49.4,48.8,37.9,37.2,37.1,36.6,32.7,32.2,31.9,30.0,29.7,29.6,29.5,29.5,29.3,29.3,29.2,28.8$, $27.1,25.5,23.6,23.2,23.1,22.7,21.3,21.0,20.9,20.8,20.7,20.7,20.6,20.5,20.5,19.7,14.1 . \mathrm{m} / \mathrm{z}$ (MALDI): found $[\mathrm{M}+\mathrm{Na}]^{+} 3709.15, \mathrm{C}_{184} \mathrm{H}_{239} \mathrm{~N}_{5} \mathrm{O}_{73}$ calcd for $[\mathrm{M}+\mathrm{Na}]^{+} 3709.50$. 


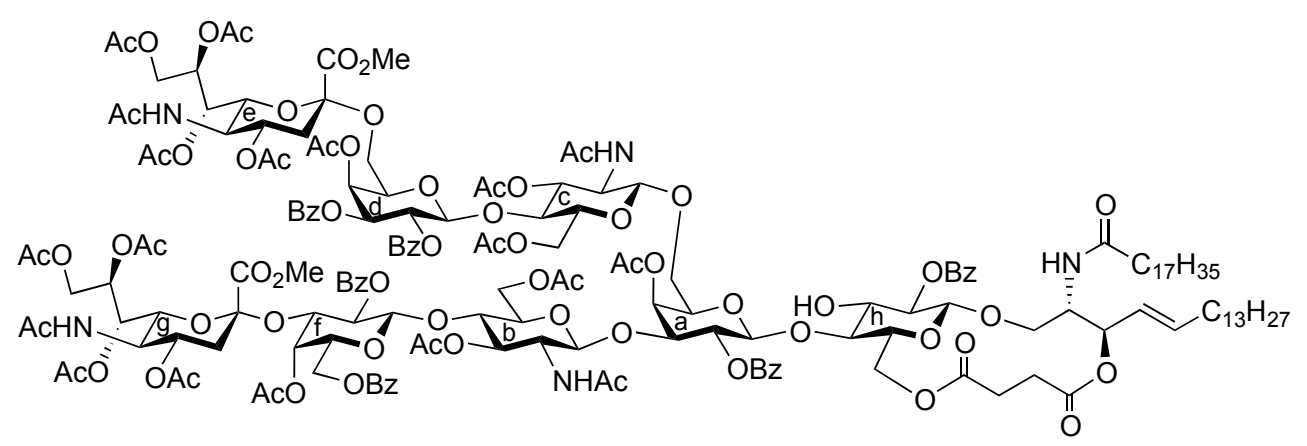

(2S,3R,4E)-1-O-(\{(Methyl 5-acetamido-4,7,8,9-tetra-O-acetyl-3,5-dideoxy-D-glycero- $\alpha$-D-galacto-2nonulopyranosylonate)-(2 $\rightarrow 3)$-4-O-acetyl-2,6-di-O-benzoyl- $\beta$-D-galactopyranosyl-( $1 \rightarrow 4)$-2-acetamido3,6-di-O-acetyl-2-deoxy- $\beta$-D-glucopyranosyl- $(1 \rightarrow 3)$-[(methyl 5-acetamido-4, 7,8,9-tetra-O-acetyl-3,5dideoxy-D-glycero- $\alpha$-D-galacto-2-nonulopyranosylonate)-(2 $\rightarrow 6)-4-O$-acetyl-2,3-di-O-benzoyl- $\beta$ - $D$ galactopyranosyl-( $(\rightarrow 4)$-2-acetamido-3,6-di-O-acetyl-2-deoxy- $\beta$-D-glucopyranosyl- $(1 \rightarrow 6)]$-4-O-acetyl2-O-benzoyl- $\beta$-D-galactopyranosyl\}-( $1 \rightarrow 4)$-2-O-benzoyl- $\beta$-D-glucopyranosyl)-2-octadecanamido-3, 6'succinyl-octadec-4-ene-1,3-diol (40). To a solution of $39(9.0 \mathrm{mg}, 2.47 \mu \mathrm{mol})$ in $\mathrm{CH}_{2} \mathrm{Cl}_{2}(0.60 \mathrm{~mL})$ was added TFA $(0.30 \mathrm{~mL})$ at $0{ }^{\circ} \mathrm{C}$. After stirring for $30 \mathrm{~min}$ at $0{ }^{\circ} \mathrm{C}$ as the reaction was monitored by TLC $\left(10: 1 \mathrm{CHCl}_{3}-\mathrm{MeOH}\right)$, the reaction was quenched by the addition of satd aq $\mathrm{NaHCO}_{3}$. The mixture was diluted with $\mathrm{CHCl}_{3}$ and washed with brine. The organic layer was subsequently dried over $\mathrm{Na}_{2} \mathrm{SO}_{4}$, and concentrated. The residue was purified by silica gel column chromatography (20:1 $\left.\mathrm{CHCl}_{3}-\mathrm{MeOH}\right)$ to give 40 (8.0 mg, 91\%). $[\alpha]_{\mathrm{D}}-6.3^{\circ}\left(\mathrm{c} 0.8, \mathrm{CHCl}_{3}\right) ;{ }^{1} \mathrm{H}-\mathrm{NMR}\left(600 \mathrm{MHz}, \mathrm{CDCl}_{3}\right) \delta$ 8.17-7.27 (m, $30 \mathrm{H}, 6 \mathrm{Ph}), 6.68\left(\mathrm{~d}, 1 \mathrm{H}, J_{2, \mathrm{NH}}=8.2 \mathrm{~Hz}, \mathrm{NH}^{C e r}\right), 6.21\left(\mathrm{~d}, 1 \mathrm{H}, J_{2, \mathrm{NH}}=9.0 \mathrm{~Hz}, \mathrm{NHb}\right)$, 5.85 (near dt, $1 \mathrm{H}, J_{5,6}=6.9 \mathrm{~Hz}, J=14.4 \mathrm{~Hz}, J=7.5 \mathrm{~Hz}, \mathrm{H}-5^{C e r}$ ), 5.70 (d, $1 \mathrm{H}, J_{3,4}=3.4 \mathrm{~Hz}, \mathrm{H}-4 \mathrm{~d}$ ), 5.59 (m, $1 \mathrm{H}, \mathrm{H}-8 \mathrm{~g}$ ), 5.57 (near t, $1 \mathrm{H}, J_{1,2}=8.3 \mathrm{~Hz}, J_{2,3}=10.3 \mathrm{~Hz}, \mathrm{H}-2 \mathrm{~d}$ ), 5.45 (dd, $1 \mathrm{H}, \mathrm{H}-3 \mathrm{~d}$ ), 5.41-5.38 (m, 1 H, H-8e), 5.35-5.32 (m, 2 H, H-2a, H-7e), 5.27-5.17 (m, 7 H, H-4a, H-3b, H-2f, H-7g, NHe, $\mathrm{H}-3^{C e r}, \mathrm{H}-4^{C e r}$ ), 5.09 (d, $1 \mathrm{H}, J_{3,4}=2.7 \mathrm{~Hz}, \mathrm{H}-4 \mathrm{f}$ ), 5.06 (near t, $1 \mathrm{H}, J_{1,2}=4.8 \mathrm{~Hz}, J_{2,3}=5.5 \mathrm{~Hz}, \mathrm{H}-2 \mathrm{~h}$ ), $4.94\left(\mathrm{~d}, 1 \mathrm{H}, J_{5, \mathrm{NH}}=10.3 \mathrm{~Hz}, \mathrm{NHg}\right), 4.91-4.81$ (m, 5 H, H-3c, H-1d, H-4e, H-4g, NHc), 4.74-4.70 (m, 2 H, H-1a, H-3f), 4.62 (d, 1 H, H-1h), 4.45-4.34 (m, 6 H, H-1b, H-1c, H-9e, H-6f, H-9g, H-6h), 4.27-4.23 (m, 3 H, H-2 ${ }^{\text {cer }}$, H-6a, H-6d), 4.17-3.98 (m, 11 H, H-6'a, H-6c, H-6'c, H-6d, H-5e, H-6e, H-9'e, H-9'g, H-5h, H-6h, H-6'h), 3.88-3.65 (m, 17 H, H-3a, H-2b, H-4b, H-6b, H-6'b, H-2c, H-4c, H-5g, H-3h, H-4h, $\mathrm{H}-1^{\mathrm{Cer}}, 2 \mathrm{OCH}_{3}$ ), 3.60-3.53 (m, 3 H, H-5d, H-6g, H-1 ${ }^{\mathrm{Cer}}$ ), 3.50-3.44 (m, 2 H, H-5b, H-5f), 3.29-3.23 (m, 2 H, H-5a, H-5c), 2.56-2.39 (m, 6 H, H-3eeq, H-3geq, $\left.2 \mathrm{C}(=\mathrm{O}) \mathrm{CH}_{2}\right), 2.20-1.78$ (m, $56 \mathrm{H}, 17$ Ac, $\left.\mathrm{H}-3 \mathrm{e} a x, \mathrm{H}-6^{\mathrm{Cer}}, \mathrm{H}-6^{\prime C e r}, \mathrm{C}(=\mathrm{O}) \mathrm{CH}_{2}{ }^{C e r}\right), 1.62\left(\mathrm{t}, 1 \mathrm{H}, J_{\mathrm{gem}}=J_{3 \mathrm{ax}, 4}=12.6 \mathrm{~Hz}, \mathrm{H}-3 \mathrm{gax}\right), 1.54(\mathrm{~s}, 3 \mathrm{H}, \mathrm{Ac})$, 1.51-1.46 (m, $\left.2 \mathrm{H}, \mathrm{C}(=\mathrm{O}) \mathrm{CH}_{2} \mathrm{CH}_{2}\right), 1.42(\mathrm{~s}, 3 \mathrm{H}, \mathrm{Ac}), 1.38-1.20\left(\mathrm{~m}, 50 \mathrm{H}, 25-\mathrm{CH}_{2}-\right), 0.88(\mathrm{~m}, 6 \mathrm{H}$, $\left.2-\mathrm{CH}_{3}{ }^{\mathrm{Cer}}\right) ;{ }^{13} \mathrm{C}-\mathrm{NMR}\left(150 \mathrm{MHz}, \mathrm{CDCl}_{3}\right) \delta 172.9,171.0,170.9,170.8,170.8,170.7,170.6,170.5$, $170.5,170.3,170.3,170.3,170.1,170.1,170.0,169.9,169.8,169.7,169.7,168.0,167.8,165.7,165.4$, $165.3,165.2,165.0,164.7,138.5,134.0,133.5,133.3,133.2,130.3,129.8,129.7,129.7,129.6,129.5$, $129.4,129.0,129.0,128.7,128.6,128.5,128.4,128.4,128.4,125.0,101.7,101.2,100.7,99.8,99.6$, $99.3,98.5,96.8 .82 .0,77.5,75.2,74.5,74.2,73.8,73.5,72.9,72.7,72.6,72.5,72.0,71.9,71.7,71.2$, $71.0,70.9,70.7,70.1,69.9,69.6,69.4,68.6,67.8,67.6,67.4,67.2,67.1,66.6,66.3,62.9,69.4,68.6$, $67.8,67.6,67.4,67.2,67.1,66.6,66.3,62.9,62.5,62.4,62.0,61.1,60.9,54.2,53.9,53.0,53.0,50.1$, $49.5,48.8,37.8,37.2,36.4,32.2,31.9,30.4,29.7,29.7,29.7,29.6,29.5,29.5,29.3,29.2,28.8,25.6$, 23.6, 23.2, 23.1, 22.7, 22.5, 21.4, 21.1, 20.8, 20.8, 20.7, 20.6, 20.5, 20.4, 14.1. $\mathrm{m} / \mathrm{z}$ (MALDI): found 
$[\mathrm{M}+\mathrm{Na}]^{+}$3589.60, $\mathrm{C}_{176} \mathrm{H}_{231} \mathrm{~N}_{5} \mathrm{O}_{72}$ calcd for $[\mathrm{M}+\mathrm{Na}]^{+}$3589.45. HRMS (ESI): found [1/2M+Na] 1806.2182, $\mathrm{C}_{176} \mathrm{H}_{231} \mathrm{~N}_{5} \mathrm{O}_{72}$ calcd for $[1 / 2 \mathrm{M}+\mathrm{Na}]^{+} 1806.2181$.

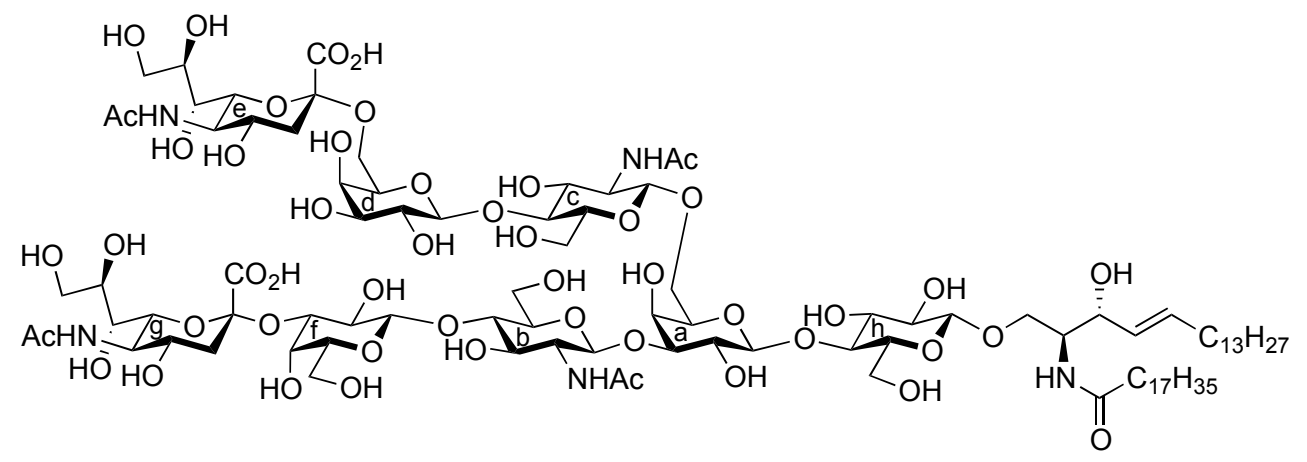

$\{3-O-[(5-A c e t a m i d o-3,5-d i d e o x y-D-g l y c e r o-\alpha$-D-galacto-2-nonulopyranosylonic $\quad$ acid $)-\beta$ - $D$ galactopyranosyl-( $1 \rightarrow 4)$-2-acetamido-2-deoxy- $\beta$-D-glucopyranosyl]-6-O-[(5-acetamido-3,5-dideoxyD-glycero- $\alpha$-D-galacto-2-nonulopyranosylonic acid)- $\beta$-D-galactopyranosyl-( $1 \rightarrow 4)$-2-acetamido-2deoxy- $\beta$-D-glucopyranosyl]- $\beta$-D-galactopyranosyl\}-( $1 \rightarrow 4)$ - $\beta$-D-glucopyranosyl- $(1 \rightarrow 1)-(2 S, 3 R, 4 E)-2$ octadecanamido-4-octadecene-1,3-diol (1). To a solution of 40 (8.0 mg, $2.24 \mu \mathrm{mol})$ in $\mathrm{MeOH} / \mathrm{THF}$ $(1: 1,1.0 \mathrm{~mL})$ was added $\mathrm{NaOMe}(28 \%$ solution in $\mathrm{MeOH}, 12.1 \mu \mathrm{g}, 0.672 \mu \mathrm{mol})$ at $0{ }^{\circ} \mathrm{C}$. After stirring for $7 \mathrm{~d}$ at $40{ }^{\circ} \mathrm{C}$ as the reaction was monitored by TLC $\left(6: 4: 1 \mathrm{CHCl}_{3}-\mathrm{MeOH}-0.2 \%\right.$ aq $\left.\mathrm{CaCl}_{2}\right)$ and MALDI-TOF MS, water $(0.1 \mathrm{~mL})$ was added to the reaction mixture. After stirring for $2 \mathrm{~d}$ at $40{ }^{\circ} \mathrm{C}$, the reaction was neutralized with Dowex $\left(\mathrm{H}^{+}\right)$resin. The resin was filtered through cotton and the filtrate was then evaporated. The residue was purified by gel filtration column chromatography (LH-20) using $\mathrm{MeOH}$ as eluent followed by silica gel column chromatography $\left(6: 4: 1 \mathrm{CHCl}_{3}-\mathrm{MeOH}-\mathrm{H}_{2} \mathrm{O}\right)$ to give $1(3.0 \mathrm{mg}, 61 \%)$. $[\alpha]_{\mathrm{D}}-78.8^{\circ}(\mathrm{c} 0.4, \mathrm{MeOH}) ;{ }^{1} \mathrm{H}-\mathrm{NMR}\left(600 \mathrm{MHz}, 1: 1 \mathrm{CD}_{3} \mathrm{OD}-\mathrm{D}_{2} \mathrm{O}\right) \delta 5.73(\mathrm{~m}$, $\left.1 \mathrm{H}, \mathrm{H}-5^{C e r}\right), 5.46$ (m, $\left.1 \mathrm{H}, \mathrm{H}-4^{C e r}\right), 2.86$ (dd, $\left.1 \mathrm{H}, \mathrm{H}-3 \mathrm{geq}\right), 2.78$ (dd, $\left.1 \mathrm{H}, \mathrm{H}-3 \mathrm{eeq}\right), 2.20$ (t, $2 \mathrm{H}$, $\mathrm{NHCOCH}_{2}$ ), 2.04 (s, 14 H, H-6 $\left.{ }^{\mathrm{Cer}}, \mathrm{H}_{-} 6^{\mathrm{Cer}}, 4 \mathrm{NAc}\right), 1.73$ (m, 2 H, H-3gax, H-3eax), 1.58 (m, 2 H, $\left.\mathrm{NHCOCH}_{2} \mathrm{CH}_{2}\right), 1.30\left(\mathrm{~m}, 50 \mathrm{H}, 25-\mathrm{CH}_{2}-\right), 0.92\left(\mathrm{t}, 6 \mathrm{H}, 2-\mathrm{CH}_{3}\right) ;{ }^{13} \mathrm{C}-\mathrm{NMR}(125 \mathrm{MHz}, 5: 6: 0.5$ $\left.\mathrm{CD}_{3} \mathrm{OD}-\mathrm{D}_{2} \mathrm{O}-\mathrm{CDCl}_{3}\right) \delta 174.2,174.1,173.7,173.3,134.1,128.6,102.9,102.3,102.0,101.8,99.9$, 79.9, 79.0, 77.8, 76.5, 75.1, 74.6, 74.1, 74.0, 73.5, 73.1, 72.5, 72.2, 72.0, 71.7, 71.6, 71.1, 70.9, 70.6, 70.2, $69.3,68.7,68.2,67.9,67.8,67.5,67.3,66.8,62.4,62.2,60.4,59.7,54.6,54.5,52.4,51.6,51.5,48.1,46.7$, 39.7, 39.4, 35.6, 31.6, 31.1, 31.0, 29.0, 28.8, 28.7, 28.6, 28.6, 28.5, 28.4, 25.3, 21.8, 21.6, 21.4, 21.2, 21.1, 12.9. HRMS (ESI): found [M+Na] $2225.0943, \mathrm{C}_{98} \mathrm{H}_{171} \mathrm{~N}_{5} \mathrm{O}_{49}$ calcd for $[\mathrm{M}+\mathrm{Na}]^{-} 2225.0940$.

\subsection{Materials and Methods for Binding Assay}

\subsubsection{Virus Preparation}

A/Memphis/1/71 (H3N2) and A/Puerto Rico/8/34 (H1N1) were used in this study. The virus was propagated in 11 days old embryonated hen's eggs. The virus was purified by ultra-centrifugation and stored at $-80{ }^{\circ} \mathrm{C}$ before use.

\subsubsection{Solid-Phase Binding Assay}

Virus binding to sialylglycolipids was determined according to a method described previously [28]. Synthetic and authentic gangliosides were 2-fold serially diluted in $100 \%$ ethanol from 0.625 to 
$2.5 \mathrm{pmol} / \mu \mathrm{L}$. Ten $\mu \mathrm{L}$ of each diluted ganglioside was placed into a well of a polystyrene Universal-BIND ${ }^{\mathrm{TM}}$ microplates (flat-bottom, Product\# 2503, Corning, Tokyo, Japan) and incubated for approximately $1 \mathrm{~h}$ at $37{ }^{\circ} \mathrm{C}$ until the ethanol had completely evaporated. gangliosides were covalently immobilized on the surface of plates by exposure for 1 min under ultraviolet irradiation $(254 \mathrm{~nm})$ according to the manufacture's instruction. Each well was blocked for $1 \mathrm{~h}$ at room temperature with PBS containing $2 \%$ bovine serum albumin. After 3 washes with PBS, the plates were incubated in solutions containing viruses in PBS (128 HA unit/50 $\mu \mathrm{L} /$ well) overnight at $4{ }^{\circ} \mathrm{C}$. After 5 washes with ice-cold PBS, the plates were incubated in a substrate solution containing $40 \mu \mathrm{M}$ 2'-(4-methylumbelliferyl)- $\alpha$-D- $N$-acetylneuraminic acid in PBS $\left(50 \mu \mathrm{L} /\right.$ well) at $37{ }^{\circ} \mathrm{C}$ for $60 \mathrm{~min}$ to detect virus neuraminidase (NA) activity associated with bound viruses. The reactions were terminated by addition of $500 \mathrm{mM}$ carbonate buffer $(\mathrm{pH} 10.2)(50 \mu \mathrm{L} /$ well $)$. Fluorescence intensity of the 4-methylumbelliferone released by viral NAs was measured at $355 \mathrm{~nm}$ (excitation) and $460 \mathrm{~nm}$ (emission). Triplicate measurements were performed in each assay. The direct virus binding activity was calculated as follows: Virus-binding score $=[$ (average of NA activity of triplicate gangliosideimmobilized wells) - (average of NA activity of triplicate ethanol-treated wells)]/[NA activity of applied virus (128 HA units $/ 50 \mu \mathrm{L})]$. The virus-binding score was expressed as mean score $\pm \mathrm{SD}$.

\section{Conclusions}

We efficiently synthesized a novel ganglioside designed as a ligand for influenza A viruses employing the cassette coupling approach between the heptasaccharyl sugar part and the cyclic glucosylceramide moiety. The present study revealed that the cassette approach can be applied to the synthesis of lacto-series gangliosides as well as ganglio-series gangliosides. This success will expand the applicability of the cassette approach to the synthesis of other glycolipids. In addition, we examined the binding activity of the synthesized ganglioside ligand to influenza A viruses. It was found that the synthetic ligand is recognized by Neua2-3 and 2-6 type viruses, suggesting that a glycan

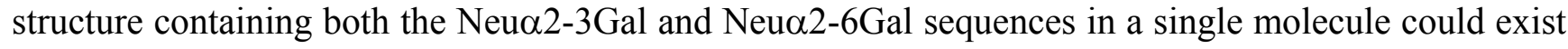
as a natural ligand for influenza A viruses. To identify an actual natural ligand for influenza viruses,

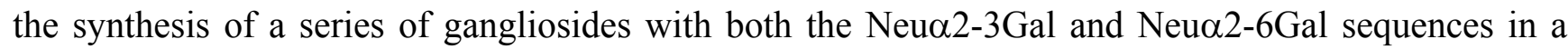
single molecule is currently underway.

\section{Acknowledgments}

This work was financially supported by the Ministry of Education, Culture, Sports, Sciences, and Technology (MEXT) of Japan (WPI program and Grant-in-Aid for Scientific Research to M.K., No.17101007 and 22380067).

\section{References}

1. Kilbourne, E.D. Influenza pandemics of the 20th Century. Emerg. Infect. Dis. 2006, 12, 9-14.

2. Hashimoto, T.; Kawaoka, Y. Influenza: Lessons from past pandemics, warnings from current incidents. Nat. Rev. Microbiol. 2005, 3, 591-600. 
3. Suzuki, Y. Sialobiology of influenza molecular mechanism of host range variation of influenza viruses. Biol. Pharm. Bull. 2005, 28, 399-408.

4. Wilks, S.; de Graaf, M.; Smith, D.J.; Burke, D.F. A review of influenza hemagglutinin receptor binding as it relates to pandemic properties. Vaccine 2012, 30, 4369-4376.

5. Fujikawa, K.; Imamura, A.; Ishida, H.; Kiso, M. Synthesis of a GM3 ganglioside analogue carrying a phytoceramide moiety by intramolecular glycosylation as a key step. Carbohydr. Res. 2008, 343, 2729-2734.

6. Imamura, A.; Ando, H.; Ishida, H.; Kiso, M. Ganglioside GQ1b: Efficient total synthesis and the expansion to synthetic derivatives to elucidate its biological roles. J. Org. Chem. 2009, 74, 3009-3023.

7. Fujikawa, K.; Nohara, T.; Imamura, A.; Ando, H.; Ishida, H.; Kiso, M. A cyclic glucosyl ceramide acceptor as a versatile building block for complex ganglioside synthesis. Tetrahedron Lett. 2010, $51,1126-1130$.

8. Fujikawa, K.; Nakashima, S.; Konishi, M.; Fuse, T.; Komura, N.; Ando, T.; Ando, H.; Yuki, N.; Ishida, H.; Kiso, M. The first total synthesis of ganglioside GalNAc-GD1a, a target molecule for autoantibodies in Guillain-Barré syndrome. Chem. Eur. J. 2011, 17, 5641-5651.

9. Tamai, H.; Ando, H.; Tanaka, H.-N.; Hosoda-Yabe, R.; Yabe, T.; Ishida, H.; Kiso, M. The total synthesis of the neurogenic ganglioside LLG-3 isolated from the starfish Linckia laevigata. Angew. Chem. Int. Ed. 2011, 50, 2330-2333.

10. Nakashima, S.; Ando, H.; Saito, R.; Tamai, H.; Ishida, H.; Kiso, M. Efficiently synthesizing lacto-ganglio-series gangliosides by using a glucosyl ceramide cassette approach: The total synthesis of ganglioside X2. Chem. Asian J. 2012, 7, 1041-1051. Part 156 of the synthetic studies on sialoglycoconjugates.

11. Fuse, T.; Ando, H.; Imamura, A.; Sawada, N.; Ishida, H.; Kiso, M.; Ando, T.; Li, S.-C.; Li, Y.-T. Synthesis and enzymatic susceptibility of a series of novel GM2 analogs. Glycoconj. J. 2006, 23, 329-343.

12. Imamura, A.; Yoshikawa, T.; Komori, T.; Ando, M.; Ando, H.; Wakao, M.; Suda, Y.; Ishida, H.; Kiso, M. Design and synthesis of versatile ganglioside probes for carbohydrate microarrays. Glycoconj. J. 2008, 25, 269-278.

13. Veeneman, G.H.; van Leeuwen, S.H.; van Boom, J.H. Iodonium ion promoted reactions at the anomeric centre. II An efficient thioglycoside mediated approach toward the formation of 1,2-trans linked glycosides and glycosidic esters. Tetrahedron Lett. 1990, 31, 1331-1334.

14. Konradosson, P.; Udodong, U.E.; Fraser-Reid, B. Iodonium promoted reactions of disarmed thioglycoside. Tetrahedron Lett. 1990, 31, 4313-4316.

15. Kanie, O.; Kiso, M.; Hasegawa, A. Glycosylation using methylthioglycosides of $N$-acetylneuraminic acid and dimethyl(methylthio)sulfonium triflate. J. Carbohydr. Chem. 1988, 7, 501-506.

16. Murase, T.; Kameyama, A.; Kartha, K.P.R.; Ishida, H.; Kiso, M.; Hasegawa, A. Synthetic studies on sialoglycoconjugates. 5: A facile, regio and stereoselective synthesis of ganglioside GM4 and its position isomer. J. Carbohydr. Chem. 1989, 8, 265-283.

17. Cook, A.F. The use of $\beta, \beta, \beta$-tribromoethyl chloroformate for the protection of nucleoside hydroxyl groups. J. Org. Chem. 1968, 33, 3589-3593. 
18. Matsuzaki, Y.; Ito, Y.; Nakahara, Y.; Ogawa, T. Synthesis of branched poly- $N$-acetyl-lactosamine type pentaantennary pentacosasaccharide: Glycan part of a glycosyl ceramide from rabbit erythrocyte membrane. Tetrahedron Lett. 1993, 34, 1061-1064.

19. Schmidt, R.R. New methods for the synthesis of glycosides and oligosaccharides-Are there alternatives to the Koenigs-Knorr method? [new synthetic methods (56)]. Angew. Chem. Int. Ed. 1986, 25, 212-235.

20. Hatakeyama, S.; Mori, H.; Kitano, K.; Yamada, H.; Nishizawa, M. Efficient reductive etherification of carbonyl compounds with alkoxytrimethylsilanes. Tetrahedron Lett. 1994, 35, 4367-4370.

21. Debenham, S.D.; Toone, E.J. Regioselective reduction of 4,6- $O$-benzylidenes using triethylsilane and $\mathrm{BF}_{3} \cdot \mathrm{Et}_{2} \mathrm{O}$. Tetrahedron: Asymmetry 2000, 11, 385-387.

22. Jansson, K.; Ahlfors, S.; Frejd, T.; Kihlberg, J.; Magnusson, G.; Dahmen, J.; Noori, G.; Stenvall, K. 2-(Trimethylsilyl)ethyl glycosides. 3. Synthesis, anomeric deblocking, and transformation into 1,2-trans 1-O-acyl sugars. J. Org. Chem. 1988, 53, 5629-5647.

23. Sakakura, A.; Kawajiri, K.; Ohkubo, T.; Kosugi, Y.; Ishihara, K. Widely useful DMAP-catalyzed esterification under auxiliary base- and solvent-free conditions. J. Am. Chem. Soc. 2007, 129, $14775-14779$.

24. Lefeber, D.J.; Kamerling, J.P.; Vliegenthart, J.F.G. The use of diazabicyclo[2.2.2]octane as a novel highly selective dechloroacetylation reagent. Org. Lett. 2000, 2, 701-703.

25. Koeners, H.J.; Verhoeven, J.; van Boom, J.H. Application of levulinic acid ester as a protecting group in the synthesis of oligosaccharides. Rec. Trav. Chim. Pays-Bas. 1981, 100, 65-72.

26. Fukunaga, K.; Toyoda, T.; Ishida, H.; Kiso, M. Synthesis of lacto- and neolacto-series ganglioside analogs containing $\mathrm{N}$-glycolylneuraminic acid: Probes for investigation of specific receptor structures recognized by influenza A viruses. J. Carbohydr. Chem. 2003, 22, 919-937.

27. Benakli, K.; Zha, C.; Kerns, R.J. Oxazolidinone protected 2-amino-2-deoxy-D-glucose derivatives as versatile intermediates in stereoselective oligosaccharide synthesis and the formation of $\alpha$-linked glycosides. J. Am. Chem. Soc. 2001, 123, 9461-9462.

28. Hidari, K.I.P.J.; Murata, T.; Yoshida, K.; Takahashi, Y.; Minamijima, Y.H.; Miwa, Y.; Adachi, S.; Ogata, M.; Usui, T.; Suzuki, Y.; et al. Chemoenzymatic synthesis, characterization, and application of glycopolymers carrying lactosamine repeats as entry inhibitors against influenza virus infection. Glycobiology 2008, 18, 779-788.

Sample Availability: Not available.

(C) 2012 by the authors; licensee MDPI, Basel, Switzerland. This article is an open access article distributed under the terms and conditions of the Creative Commons Attribution license (http://creativecommons.org/licenses/by/3.0/). 